\title{
Learning the intensity of time events with change-points
}

\author{
Mokhtar Z. Alaya ${ }^{1} \quad$ Stéphane Gaïffas ${ }^{2} \quad$ Agathe Guilloux $^{3}$
}

July 3, 2015

\begin{abstract}
We consider the problem of learning the inhomogeneous intensity of a counting process, under a sparse segmentation assumption. We introduce a weighted totalvariation penalization, using data-driven weights that correctly scale the penalization along the observation interval. We prove that this leads to a sharp tuning of the convex relaxation of the segmentation prior, by stating oracle inequalities with fast rates of convergence, and consistency for change-points detection. This provides first theoretical guarantees for segmentation with a convex proxy beyond the standard i.i.d signal + white noise setting. We introduce a fast algorithm to solve this convex problem. Numerical experiments illustrate our approach on simulated and on a high-frequency genomics dataset.
\end{abstract}

Keywords. Counting processes, Total-variation, Oracle inequalities, Change-points

\section{Introduction}

Counting processes are widely used in engineering to describe systems where stochastic events occur, such as genomics, biology, econometrics, communications and networks, see [2]. In these problems, the aim is to estimate the intensity function, which determines the instantaneous rate of occurrence of an event. In the statistical literature, this topic has been extensively discussed in several previous works. Procedures based on kernel estimation [29], cross-validation [20], wavelet methods [26], local polynomial estimators [13], model selection [30] are considered for the non-parametric estimation of the intensity.

In this paper, we want to recover the intensity $\lambda_{0}(t)$ of a counting process $\{N(t), t \in$ $[0,1]\}$ from $n$ observations of $N$. We work under the assumption that $\lambda_{0}$ can be wellapproximated by a piecewise constant function, and we deal with this problem with a signal segmentation point-of-view, where the goal is to find the unknown times of abrupt changes in the dynamic of the signal. This is referred to multiple change-point problem in statistical literature, see [24] for a recent review with interesting references. A changepoint is a time or position where the structure of the object changes and the goal of change-point detection is to estimate these positions.

Several examples of practical importance fulfill the model of multiple change-points. A particularly interesting example comes from the next-generation sequencing (NGS) DNA

\footnotetext{
${ }^{1}$ Sorbonne Universités, UPMC Univ Paris 06, F-75005, Paris, France, email: elmokhtar .alaya@upmc.fr

${ }^{2}$ Centre de Mathématiques Appliquées, École Polytechnique, and CNRS UMR 7641, 91128 Palaiseau, France, email: stephane.gaiffas@cmap.polytechnique.fr

${ }^{3}$ Sorbonne Universités, UPMC Univ Paris 06, F-75005, Paris, and Unité INSERM 762 "Instabilité des Microsatellites et Cancers", France email: agathe.guilloux@upmc.fr
} 
process. Indeed, an important application of NGS technologies is the study of the transcriptome and the resulting experiment is called RNA-seq. In a typical RNA-seq experiment, a sample of RNA is amplified, shattered, and converted to a library of a cDNA fragments. Then, it is sequenced on a high-throughput platform which is available commercially. Finally, the raw data result in large amounts of DNA fragments sequences called reads. These reads are then mapped to the reference genome by an appropriate algorithm, that tells us the region from which each read comes from. RNA-seq can be modelled mathematically as replications of an inhomogeneous counting process with a piecewise constant intensity [32]. The counting process counts the number of reads whose first base maps to the left base of a given chromosome's location. In [32], a Bayesian approach for the detection of change-points is considered. Other approaches based on Bayesian model-based clustering and segmentation are given in [27].

In the present paper, we consider the estimation of $\tau_{0, \ell}$ and $\beta_{0, \ell}$ in the following model:

$$
\lambda_{0}(t)=\sum_{\ell=1}^{L_{0}} \beta_{0, \ell} \mathbb{1}_{\left(\tau_{0, \ell-1}, \tau_{0, \ell}\right]}(t)
$$

for $0 \leq t \leq 1$, with the convention $\tau_{0,0}=0$ and $\tau_{0, L_{0}}=1$. Our approach consists in reframing this task as a variable selection task. We introduce a penalized least-squares criterion with a data-driven total-variation penalization, which is $\ell_{1}$-penalization of the discrete gradient of the parameter.

This convex proxy for segmentation with an extra $\ell_{1}$-penalization for sparsity, called fused Lasso, is introduced in [33]. Theoretical guarantees for this procedure are given in [21] in the white noise setting, for the segmentation of a one-dimensional signal. A group fused Lasso is introduced in [7] for the detection of multiple change-points shared by a set of co-occurring one-dimensional signals, and an algorithm is derived to solve the corresponding convex problem. The determination of the number of structural changes in multitask learning via the group fused Lasso is considered in [28].

Beyond the one-dimensional setting, total-variation penalization is well-known and commonly used in image denoising, deblurring and segmentation, see for instance [12] and [11]. In this context, one needs to define a graph of neighboring nodes (pixels), and the problem can be solved efficiently by reformulating it as a min-cut problem and solving it using a max-flow algorithm [22].

Other close references are the following: [18] proves sharp oracle inequalities for the Lasso in hazards models, [15] studies Lasso-type estimators in a linear regression model with multiple change-points, [31] considers denoising of a sparse and block signal, [9] studies the asymptotics for jump-penalized least squares regression aiming at approximating a regression function by piecewise constant functions. An algorithm of majorizationminimization for high dimensional fused Lasso regression is proposed in [35], a testing approach for the segmentation of the hazard function is given in [19].

The papers [30], [33], [21], [7], [28], are most relevant to our work. In [30], a model selection procedure is introduced to estimate the intensity function. In [21] and [33], the authors propose an adaptation of the Lasso algorithm to detect change-points in the standard i.i.d signal + Gaussian white noise framework. In [7] and [28], the authors use group fused Lasso to solve the structural change-points in linear regression problems. This paper is different from these works in the following aspects. First, a main feature of our results is that they are derived for a signal in continuous time, as compared to [21], [28] and [33]. Namely, we aim at detecting change-points in the intensity function. Hence, this problem is prone to an unavoidable non-parametric bias of approximation by a piecewise constant function, which makes our mathematical analysis very different. A second main 
feature of our results is that we introduce a weighted total-variation penalization, using data-driven weights that correctly scale the penalization along the observation interval. This is not necessary in the Gaussian and discrete signal + noise setting from [21] for instance. As a side product, we are able to use the same tuning parameters both for consistency in oracle inequalities, see Theorems 1 and 2, and detection of change-points, see Theorems 3 and 4. A third main feature of our approach is that we use a convex surrogate for the sparsity of the discrete gradient of the signal, that can be solved numerically very efficiently, see Section 5, even for a large signal (using many bins). This is not the case for the approach described in [30], which is based on $\ell_{0}$ model-selection techniques. Furthermore, our oracle inequalities are sharp in the sense that the leading constant in front of the bias terms is equal to one.

The rest of the paper is organized as follows. In Section 2, we provide basic notations. Then, we present our estimation procedure. Section 3 develops oracle inequalities for the estimator, see Theorems 1 and 2. Section 4 gives results in change-points detection, see Theorems 3 and 4. Section 5 describes a fast algorithm to solve the convex problem studied in the paper. The proofs of the main statements are gathered in Sections 7, 8 and 9.

\section{Counting processes with a sparse segmentation prior}

Let $(\Omega, \mathcal{F}, \mathbb{P})$ be a probability space and $\left(\mathcal{F}_{t}\right)_{0 \leq t \leq 1}$ a filtration satisfying the usual conditions [25]: increasing, right-continuous and complete. A counting process is a stochastic process $\{N(t)\}_{0 \leq t \leq 1}$ which is $\left(\mathcal{F}_{t}\right)$-adapted to the filtration, with right-continuous and piecewise constant paths almost surely (a.s.), with jump of size +1 at event times such that $N(0)=0$ and $N(t)<\infty$ a.s. The term counting process is natural: $N(t)-N(s)$ corresponds to the number of events of a certain type occurring in the interval $(s, t]$. The Poisson process is the most common example of a counting process, where the jumps occur randomly and independently of each other on disjoint intervals, see for instance [10] and [23] for references on point processes and their statistical estimation.

Since $N$ is increasing, it is a submartingale, so it follows from the Doob-Meyer decomposition theorem [1]. Namely, $N=\Lambda_{0}+M$, where $\Lambda_{0}$ is a predictable increasing process called the compensator of $N$ and $M$ is a $\left(\mathcal{F}_{t}\right)$-martingale. We assume in the following that

$$
\Lambda_{0}(t)=\mathbb{E}[N(t)]=\int_{0}^{t} \lambda_{0}(s) d s
$$

for $0 \leq t \leq 1$, where $\lambda_{0}$ is a non-negative right-continuous function with left-hand limits called intensity rate of $N$. Under this assumption, $M(t)=N(t)-\int_{0}^{t} \lambda_{0}(s) d s$ is a local square-integrable martingale with quadratic variation given by $\langle M\rangle(t)=\int_{0}^{t} \lambda_{0}(s) d s$ and optional variation $[M](t)=\int_{0}^{t} \lambda_{0}(s) d N(s)$.

\subsection{Sparse segmentation assumption}

We work under the assumption that the intensity is piecewise constant, over unknown inhomogeneous intervals of time. From now on, $\mathbb{1}_{A}$ stands for the indicator function of a set $A$. For some results in the paper, we will use

Assumption 1. We assume that the intensity writes

$$
\lambda_{0}(t)=\sum_{\ell=1}^{L_{0}} \beta_{0, \ell} \mathbb{1}_{J_{\ell}}(t), 0 \leq t \leq 1,
$$


with $L_{0} \geq 1, \beta_{0, \ell}$ are positive coefficients, and where $J_{0}=\{0\}, J_{\ell}=\left(\tau_{0, \ell-1}, \tau_{0, \ell}\right]$ for $\ell=1, \ldots, L_{0}$ and $\tau_{0,0}=0<\tau_{0,1}<\cdots<\tau_{0, L_{0}-1}<\tau_{0, L_{0}}=1$.

Assumption 1 means that $L_{0}-1$ changes affect the value of $\lambda_{0}$ at unknown instants $\tau_{0, \ell}$. The number of change-points $L_{0}-1$ is unknown. In this setting, we want to recover the intensity $\lambda_{0}$, by jointly estimating $L_{0}, \tau_{0, \ell}$ and $\beta_{0, \ell}$, for $\ell=1, \ldots, L_{0}-1$. Throughout the paper, we will assume the following.

Assumption 2. We observe $n$ i.i.d copies of $N$ on $[0,1]$, denoted $N_{1}, \ldots, N_{n}$.

The assumption that the process is in $[0,1]$ is for the sake of simplicity. Assumption 2 is equivalent to observing a single process $N$ with intensity $n \lambda_{0}$, which is only used to have a notion of growing observations with an increasing $n$.

\subsection{A procedure based on total-variation penalization}

Fix $m=m_{n} \geq 1$, an integer that shall go to infinity as $n \rightarrow \infty$. Let us define the set of nonnegative piecewise constant functions on $[0,1]$ given by

$$
\Lambda_{m}=\left\{\lambda_{\beta}=\sum_{j=1}^{m} \beta_{j, m} \lambda_{j, m}: \beta=\left[\beta_{j, m}\right]_{1 \leq j \leq m} \in \mathbb{R}_{+}^{m}\right\},
$$

where

$$
\lambda_{j, m}=\sqrt{m} \mathbb{1}_{I_{j, m}} \quad \text { and } \quad I_{j, m}=\left(\frac{j-1}{m}, \frac{j}{m}\right] .
$$

The linear space $\Lambda_{m}$ is endowed by the norm $\|\lambda\|=\left(\int_{0}^{1} \lambda^{2}(t) d t\right)^{1 / 2}$. We introduce the least-squares functional

$$
R_{n}(\lambda)=\int_{0}^{1} \lambda(t)^{2} d t-\frac{2}{n} \sum_{i=1}^{n} \int_{0}^{1} \lambda(t) d N_{i}(t)
$$

which is the goodness-of-fit criterion to be used in this setting, see among others [30]. Note that $\left\{\lambda_{j, m}: j=1, \ldots, m\right\}$ produces an orthonormal basis of $\Lambda_{m}$, it implies that

$$
R_{n}\left(\lambda_{\beta}\right)=\sum_{j=1}^{m} \beta_{j, m}^{2}-\frac{2 \sqrt{m}}{n} \sum_{j=1}^{m} \sum_{i=1}^{n} \beta_{j, m} N_{i}\left(I_{j, m}\right)
$$

for any $\beta \in \mathbb{R}_{+}^{m}$. Now, let us introduce the weighted total-variation penalization

$$
\|\beta\|_{\mathrm{TV}, \hat{w}}=\sum_{j=2}^{m} \hat{w}_{j}\left|\beta_{j}-\beta_{j-1}\right|
$$

for $\beta=\left[\beta_{j}\right]_{1 \leq j \leq m} \in \mathbb{R}^{m}$, where $\hat{w}=\left[\hat{w}_{j}\right]_{1 \leq j \leq m}$ is a positive vector of weights (eventually depending on data) to be defined later on, with $\hat{w}_{1}=0$. The data-driven weights $\hat{w}$ will allow to design sharp tuning of the total-variation penalization. Then, given $m \geq 1$ and a weights vector $\hat{w}$, we introduce

$$
\hat{\beta}=\operatorname{argmin}_{\beta \in \mathbb{R}_{+}^{m}}\left\{R_{n}\left(\lambda_{\beta}\right)+\|\beta\|_{\mathrm{TV}, \hat{w}}\right\},
$$

hence an estimator of $\lambda_{0}$ is given by $\hat{\lambda}=\lambda_{\hat{\beta}}$. An estimation of the change-point locations is obtained from the support of the discrete gradient of $\hat{\beta}$. Namely, define

$$
\hat{S}=\left\{j: \hat{\beta}_{j, m} \neq \hat{\beta}_{j-1, m} \text { for } j=2, \ldots, m\right\},
$$


and denote by $\hat{L}=|\hat{S}|$ the estimated number of change-points.

We denote the mean counting process $\bar{N}_{n}=n^{-1} \sum_{i=1}^{n} N_{i}$, and the unweighted TV penalization by $\|\beta\|_{\mathrm{TV}}=\sum_{j=2}^{m}\left|\beta_{j}-\beta_{j-1}\right|$ for $\beta \in \mathbb{R}^{m}$. We use also the notation $\bar{N}_{n}(I)=$ $\int_{I} d \bar{N}_{n}(t)$ for any $I \subset[0,1]$.

\section{Sharp oracle inequalities}

In this section we address the statistical properties of $\hat{\lambda}$ stated in (6), by proving two oracle inequalities. Theorem 1 below is an oracle inequality of "slow-type" [6] that holds in full generality, while Theorem 2 is a fast oracle inequality, that holds under the assumption that the number of the estimated change-points is upper bounded by a known constant $L_{\max }$. Both oracle inequalities are sharp in the sense that the constant term in front of the oracle term $\inf _{\beta}\left\|\lambda_{\beta}-\lambda\right\|$ is equal to one.

Theorem 1. Fix $x>0$ and introduce the data-driven weights,

$$
\hat{w}_{j}=5.66 \sqrt{\frac{m\left(x+\log m+\hat{h}_{n, x, j}\right) \hat{V}_{j}}{n}}+9.31 \frac{\sqrt{m}\left(x+1+\log m+\hat{h}_{n, x, j}\right)}{n},
$$

where $\hat{V}_{j}=\bar{N}_{n}\left(\left(\frac{j-1}{m}, 1\right]\right)$ and

$$
\hat{h}_{n, x, j}=2 \log \log \left(\frac{6 e n \hat{V}_{j}+14 e(x+\log m)}{28(x+\log m)} \vee e\right) .
$$

Then, if $\hat{\lambda}$ is given by (6), we have

$$
\left\|\hat{\lambda}-\lambda_{0}\right\|^{2} \leq \inf _{\beta \in \mathbb{R}_{+}^{m}}\left(\left\|\lambda_{\beta}-\lambda_{0}\right\|^{2}+2\|\beta\|_{\mathrm{TV}, \hat{w}}\right)
$$

with a probability larger than $1-12.85 e^{-x}$.

The proof of Theorem 1 is postponed in Section 7. We define $\beta_{0, m}=\left[\beta_{0, j, m}\right]_{1 \leq j \leq m}$ the coefficients vector of the projection of $\lambda_{0}$ on $\Lambda_{m}$ and $\Delta_{\beta, \max }=\max _{1 \leq \ell, \ell^{\prime} \leq L_{0}}\left|\beta_{0, \ell}-\beta_{0, \ell^{\prime}}\right|$, which is the maximum jump size of $\lambda_{0}$. Under Assumption 1, a control of the approximation term leads to the following.

Corollary 1. Given Assumption 1, and under the same assumptions as the ones from Theorem 1, we have

$$
\left\|\hat{\lambda}-\lambda_{0}\right\|^{2} \leq \frac{2\left(L_{0}-1\right) \Delta_{\beta, \max }^{2}}{m}+2\left\|\beta_{0, m}\right\|_{\mathrm{TV}} \max _{1 \leq j \leq m} \hat{w}_{j} .
$$

The proof of Corollary 1 is given in Section 7. Theorem 1 uses a data-driven weighting of the TV penalization, based on weights roughly given by

$$
\hat{w}_{j} \approx \sqrt{\frac{m \log m}{n} \bar{N}_{n}\left(\left(\frac{j-1}{m}, 1\right]\right) .}
$$

This exhibits a new scaling of the TV penalization, which is natural and of importance in this setting. The shape of this data-driven weighting comes from a Bernstein's concentration with data-driven variance, necessary for the control of the noise term (a martingale with jumps), given in Proposition 1 below, see Section 7.1. 
Theorem 2. Fix $x>0$ and let $\hat{\lambda}$ be the same as in Theorem 1. Assume that the estimated number of change-points $\hat{L}$ satisfies $\hat{L} \leq L_{\max }$. Then, we have

$$
\begin{gathered}
\left\|\hat{\lambda}-\lambda_{0}\right\|^{2} \leq \inf _{\beta \in \mathbb{R}_{+}^{m}}\left\|\lambda_{\beta}-\lambda_{0}\right\|^{2}+6\left(L_{\max }+2\left(L_{0}-1\right)\right) \max _{1 \leq j \leq m} \hat{w}_{j}^{2} \\
+K_{1} \frac{\left\|\lambda_{0}\right\|_{\infty}\left(x+L_{\max }(1+\log m)\right)}{n} \\
+K_{2} \frac{m\left(x+L_{\max }(1+\log m)\right)^{2}}{n^{2}}
\end{gathered}
$$

with a probability larger than $1-L_{\max } e^{-x}$, with $\left\|\lambda_{0}\right\|_{\infty}=\sup _{t \in[0,1]} \lambda_{0}(t), K_{1}=1670.89$, and $K_{2}=6683.53$.

The proof of Theorem 2 is provided in Section 7. This results proves that our procedure has a fast rate of convergence of order

$$
\frac{\left(L_{\max } \vee L_{0}\right) m \log m}{n},
$$

which scales in $m / n$.

Corollary 2. Given Assumption 1, and under the same assumptions as the ones from Theorem 2, we have

$$
\begin{aligned}
\left\|\hat{\lambda}-\lambda_{0}\right\|^{2} \leq & \frac{2\left(L_{0}-1\right) \Delta_{\beta, \max }^{2}}{m}+6\left(L_{\max }+2\left(L_{0}-1\right)\right) \max _{1 \leq j \leq m} \hat{w}_{j}^{2} \\
& +K_{1} \frac{\left\|\lambda_{0}\right\|_{\infty}\left(x+L_{\max }(1+\log m)\right)}{n} \\
& +K_{2} \frac{m\left(x+L_{\max }(1+\log m)\right)^{2}}{n^{2}}
\end{aligned}
$$

with a probability larger than $1-L_{\max } e^{-x}$, with the same notations as in Theorem 2.

The proof of Corollary 2 is presented in Section 7. A consequence of Corollary 2 is that an optimal tradeoff between approximation and complexity is given by the choice $m \approx n^{1 / 2}$. Note that we are able to use the same procedure in Theorems 1 and 2 , namely for the slow and fast rate, while it is not the case in the signal + white noise considered in [21] for instance.

\section{Change-point detection}

In this section we prove that the proposed total-variation with data-driven weights procedure is consistent for the estimation of the change-point positions. Note that, however, the context considered here is quite different from the more standard signal + white noise setting: here we aim at detecting change-points in the intensity function, hence this problem is prone to an unavoidable non-parametric bias of approximation by a piecewise constant function. This means that we will not be able to recover the exact position of two change-points if they lie on the same interval $I_{j, m}$. Therefore, we assume

Assumption 3. Grant Assumption 1 and assume that there is a positive constant $c \geq 8$ such that

$$
\min _{1 \leq \ell \leq L_{0}}\left|\tau_{0, \ell}-\tau_{0, \ell-1}\right|>\frac{c}{m} .
$$


This assumption entails that the change-points of $\lambda_{0}$ are sufficiently far apart, and that, in particular, there cannot be more than one change-point in the "high-resolution" intervals $I_{j, m}$. Under Assumption 3, the procedure will be able to recover the (unique) intervals $I_{j_{\ell}, m}$, for $\ell=0, \ldots, L_{0}$, where the change-point belongs. Hence, we define the approximate change-points sequence $\left[j_{\ell}\right]_{0 \leq \ell \leq L_{0}}$ as follows.

Definition 1. The approximate change-points sequence $\left[j_{\ell}\right]_{0 \leq \ell \leq L_{0}}$ relative to the level of resolution $m$ is defined as the right-hand side boundary of the unique interval $I_{j_{\ell}, m}$ that contains the change-point $\tau_{0, \ell}$, namely

$$
\tau_{0, \ell} \in\left(\frac{j_{\ell}-1}{m}, \frac{j_{\ell}}{m}\right]
$$

for $\ell=1, \ldots, L_{0}-1$, where we put $j_{0}=0$ and $j_{L_{0}}=m$ by convention.

Given the support $\hat{S}=\left\{\hat{j}_{1}, \ldots, \hat{j}_{\hat{L}}\right\}$ with $\hat{j}_{1}<\cdots<\hat{j}_{\hat{L}}$ of the discrete gradient of $\hat{\beta}$ defined in (7), and introducing $\hat{j}_{0}=0$ and $\hat{j}_{\hat{L}+1}=m$, we define simply

$$
\hat{\tau}_{\ell}=\frac{\hat{j}_{\ell}}{m}
$$

for $\ell=0, \ldots, \hat{L}+1$. In order to be able to prove a consistency results for change-points detection, we need a set of assumptions that quantifies the asymptotic interplay between several quantities:

- $\Delta_{j, \min }=\min _{1 \leq \ell \leq L_{0}-1}\left|j_{\ell+1}-j_{\ell}\right|$, which is the minimum distance between two consecutive terms in the change-points of $\lambda_{0}$.

- $\Delta_{\beta, \min }=\min _{1 \leq q \leq m-1}\left|\beta_{0, q+1, m}-\beta_{0, q, m}\right|$, which is the smallest jump size of the projection $\lambda_{0, m}$ of $\lambda_{0}$ onto $\Lambda_{m}$.

- $\left(\varepsilon_{n}\right)_{n \geq 1}$, a non-increasing and positive sequence that goes to zero as $n \rightarrow \infty$, and such that $m \varepsilon_{n} \geq 6$ for any $n \geq 1$.

Assumption 4. We assume that $\Delta_{j, \min }, \Delta_{\beta, \min }$ and $\left(\varepsilon_{n}\right)_{n \geq 1}$ satisfy

$$
\begin{gathered}
\frac{\sqrt{n m} \varepsilon_{n} \Delta_{\beta, \text { min }}}{\sqrt{\log m}} \rightarrow \infty \\
\frac{\sqrt{n} \Delta_{j, \min } \Delta_{\beta, \text { min }}}{\sqrt{m \log m}} \rightarrow \infty
\end{gathered}
$$

as $n \rightarrow \infty$.

This assumption controls the rate $\left(\varepsilon_{n}\right)$ of convergence of $\hat{\tau}_{\ell}$ towards $\tau_{0, \ell}$. The logarithmic factor is due to concentration inequalities for the control of the noise (the martingale $M$ obtained by compensation of $N$ ). The next Theorem proves the consistency of our procedure for the detection of change-points, under the assumption that the estimated number of change-points is the correct one.

Theorem 3. Under Assumptions 3 and 4, and if $\hat{L}=L_{0}-1$, then the change-points estimators $\left\{\hat{\tau}_{1}, \ldots, \hat{\tau}_{\hat{L}}\right\}$ given by (15) satisfy

$$
\mathbb{P}\left[\max _{1 \leq \ell \leq L_{0}-1}\left|\tau_{0, \ell}-\hat{\tau}_{\ell}\right| \leq \varepsilon_{n}\right] \rightarrow 1
$$

as $n \rightarrow \infty$. 
The proof of Theorem 3 is quite involved and is presented in Section 8 and Appendix B. It builds upon some techniques developed in [21], based on a careful inspection of the Karush-Kuhn-Tucker (KKT) optimality conditions, see for instance [8], for the solutions to the convex problem (6). The proof depends also heavily on a data-driven Bernstein's inequality for the control of the martingale errors, see Proposition 1 from Section 7.

Let us give examples of scaling for the quantities $\Delta_{j, \min }, \Delta_{\beta, \text { min }}$ and $\left(\varepsilon_{n}\right)_{n \geq 1}$ that meet Assumption 4. Assume for simplicity that

$$
\varepsilon_{n}=n^{-\alpha} \quad \text { and } \quad \Delta_{\beta, \min }=n^{-\gamma}
$$

for some constants $\alpha, \gamma>0$.

- If $m=n^{1 / 3}$ then Theorem 3 holds with any $\alpha, \gamma>0$ satisfying $0<\gamma<1 / 3$ and $0<\alpha+\gamma<2 / 3$, and if $\Delta_{j, \min } \geq 6$.

- If $m=n^{1 / 2}$ then Theorem 3 holds with any $0<\gamma<1 / 4$ and $0<\alpha+\gamma<3 / 4$ and if $\Delta_{j, \min } \geq 6$.

In order to prove change-point consistency without the assumption that the estimated number of change-points is the correct one, we need to relax a little bit the statement of the result given in Theorem 3. Namely, we evaluate a non-symmetrized Hausdorff distance $\mathcal{E}\left(\hat{\mathcal{T}} \| \mathcal{T}_{0}\right)$ between the set of estimated change-points

$$
\hat{\mathcal{T}}=\left\{\hat{\tau}_{1}, \ldots, \hat{\tau}_{\hat{L}}\right\}
$$

and the set of true change-points

$$
\mathcal{T}_{0}=\left\{\tau_{0,1}, \ldots, \tau_{0, L_{0}-1}\right\},
$$

where for two sets $A$ and $B$, the quantity $\mathcal{E}(A \| B)$ is given by

$$
\mathcal{E}(A \| B)=\sup _{b \in B} \inf _{a \in A}|a-b| .
$$

Note that $\mathcal{E}(A \| B) \vee \mathcal{E}(B \| A)$ is the Hausdorff distance between $A$ and $B$. When $\hat{L}=L_{0}-1$, Theorem 3 implies that

$$
\mathbb{P}\left[\mathcal{E}\left(\hat{\mathcal{T}} \| \mathcal{T}_{0}\right) \leq \varepsilon_{n}, \mathcal{E}\left(\mathcal{T}_{0} \| \hat{\mathcal{T}}\right) \leq \varepsilon_{n}\right] \rightarrow 1
$$

as $n \rightarrow \infty$. When $\hat{L}>L_{0}-1$, we prove in Theorem 4 below that $\mathcal{E}\left(\hat{\mathcal{T}} \| \mathcal{T}_{0}\right) \leq \varepsilon_{n}$ with a probability going to 1 as $n \rightarrow \infty$. This means that change-point consistency holds for our procedure whenever the estimated number of change-points is not less than the true one.

Theorem 4. Under Assumptions 3 and 4 , and if $\hat{L} \geq L_{0}-1$, we have

$$
\mathbb{P}\left[\mathcal{E}\left(\hat{\mathcal{T}} \| \mathcal{T}_{0}\right) \leq \varepsilon_{n}\right] \rightarrow 1
$$

as $n \rightarrow \infty$.

Theorem 4 ensures that even when the number of change-points is over-estimated, each true change-point is close to the estimated one. The proof of Theorem 4 is given in Section 9. It is based, as for the proof of Theorem 3, on a repeated utilization of the KKT optimality conditions of problem (6).

Note that a difference with [21] is that we are able to use the same regularization parameters $\hat{w}_{j}$ given by (10) in Theorems 3 and 4 . Besides, we don't need an upper bound on the estimated number of change-points in Theorem 4, while it is necessary in [28]. 


\section{Numerical experiments}

In this section we propose a fast algorithm for solving the optimization problem (6) and apply it on simulated and real datasets from genomics.

\subsection{Algorithm}

A concept of importance for convex optimization in machine learning is the proximal operator $[3,4]$. The proximal operator $\operatorname{prox}_{f}$ of a proper, lower semicontinuous, convex function $f: \mathbb{R}^{m} \rightarrow(-\infty, \infty]$, is defined as

$$
\operatorname{prox}_{f}(v)=\operatorname{argmin}_{x \in \mathbb{R}^{m}}\left\{\frac{1}{2}\|v-x\|_{2}^{2}+f(x)\right\} \text {, for all } v \in \mathbb{R}^{m} .
$$

In this section, we provide a fast algorithm to solve the optimization problem (6), that computes the proximal operator of the weighted total-variation.

We observe $n$ i.i.d observations of $N$ over the interval $[0,1]$. Recall that $\bar{N}_{n}=$ $n^{-1} \sum_{i=1}^{n} N_{i}$, and $\bar{N}_{n}(I)=\int_{I} d \bar{N}_{n}(t)$ for any $I \subset[0,1]$. We also recall that $\hat{\lambda}(t)=$ $\sum_{j=1}^{m} \hat{\beta}_{j} \lambda_{j, m}(t)$, where $\hat{\beta}=\left[\hat{\beta}_{1}, \ldots, \hat{\beta}_{m}\right]$ is given by $(6)$. Hence, we have

$$
\hat{\beta}=\operatorname{argmin}_{\beta \in \mathbb{R}_{+}^{m}}\left\{\frac{1}{2}\|\mathbf{N}-\beta\|_{2}^{2}+\|\beta\|_{\mathrm{TV}, \hat{w}}\right\},
$$

where $\mathbf{N}=\left[\mathbf{N}_{j}\right]_{1 \leq j \leq m} \in \mathbb{R}_{+}^{m}$ is given by

$$
\mathbf{N}=\left[\begin{array}{c}
\sqrt{m} \bar{N}_{n}\left(I_{1, m}\right) \\
\vdots \\
\sqrt{m} \bar{N}_{n}\left(I_{m, m}\right)
\end{array}\right]
$$

Therefore, we see that (21) is equivalent to

$$
\hat{\beta}=\operatorname{prox}_{\|\cdot\|_{\mathrm{TV}, \hat{w}}}(\mathbf{N}) .
$$

Next, we develop an algorithm that computes $\operatorname{prox}_{\|\cdot\|_{\mathrm{TV}, \hat{w}}}$, which is an extension of [17] to weighted total-variation. Towards this end, we introduce the following $(m-1) \times m$ bidiagonal matrix

$$
D_{\hat{w}}=\left[\begin{array}{ccccc}
-\hat{w}_{2} & \hat{w}_{2} & 0 & \cdots & 0 \\
0 & -\hat{w}_{3} & \hat{w}_{3} & \ddots & \vdots \\
\vdots & \ddots & \ddots & \ddots & 0 \\
0 & \cdots & 0 & -\hat{w}_{m} & \hat{w}_{m}
\end{array}\right]
$$

Then, one can express the primal problem (21) as follows:

$$
\hat{\beta}=\operatorname{argmin}_{\beta \in \mathbb{R}_{+}^{m}}\left\{\frac{1}{2}\|\mathbf{N}-\beta\|_{2}^{2}+\left\|D_{\hat{w}} \beta\right\|_{1}\right\} .
$$

Essentially, problem (22) is difficult to analyse directly because the nondifferentiable $\ell_{1}$ norm is composed with a linear transformation of $\beta$. When solving (22) we may consider its Fenchel dual form[4]. First, we rewrite the primal problem as

$$
\begin{aligned}
& \operatorname{minimize}_{\beta \in \mathbb{R}^{m}, z \in \mathbb{R}^{m-1}} \frac{1}{2}\|\mathbf{N}-\beta\|_{2}^{2}+\|z\|_{1} \\
& \text { subject to } D_{\hat{w}} \beta=z,
\end{aligned}
$$


whose Lagrangian is

$$
\mathscr{L}(\beta, z, u)=\frac{1}{2}\|\mathbf{N}-\beta\|_{2}^{2}+\|z\|_{1}+u^{\top}\left(D_{\hat{w}} \beta-z\right),
$$

and to derive a dual problem, we minimize this over $\beta, z$. A straightforward computation gives

$$
\min _{\beta}\left\{\frac{1}{2}\|\mathbf{N}-\beta\|_{2}^{2}+u^{\top} D_{\hat{w}} \beta\right\}=-\frac{1}{2}\left\|\mathbf{N}-D_{\hat{w}}^{\top} u\right\|_{2}^{2},
$$

while

$$
\min _{z}\left\{\|z\|_{1}-u^{\top} z\right\}= \begin{cases}0, & \text { if }\|u\|_{\infty} \leq 1 \\ -\infty, & \text { otherwise }\end{cases}
$$

Introducing $u_{0}=u_{m}=0$, we proved that a dual problem of (22) is given by

$$
\begin{aligned}
& \operatorname{minimize}_{u \in \mathbb{R}^{m+1}} \frac{1}{2} \sum_{k=1}^{m}\left(\mathbf{N}_{k}-\hat{w}_{k+1} u_{k}+\hat{w}_{k} u_{k-1}\right)^{2}, \\
& \text { subject to }\left|u_{j}\right| \leq 1 \text {, for } k=1, \ldots, m \text {, and } u_{0}=u_{m}=0 .
\end{aligned}
$$

If we have a feasible dual variable $\hat{u}$, we can compute the primal solution $\hat{\beta}$ using

$$
\hat{\beta}_{k}=\mathbf{N}_{k}-\hat{w}_{k+1} \hat{u}_{k}+\hat{w}_{k} \hat{u}_{k-1}, \text { for } k=1, \ldots, m .
$$

For this problem, strong duality holds, see [8], meaning that the duality gap is zero. The KKT optimality conditions characterize the unique solutions $\hat{\beta}$ and $\hat{\theta}_{k}:=\hat{w}_{k+1} \hat{u}_{k}$. They yield, in addition to (23):

$$
\hat{\theta}_{0}=\hat{\theta}_{m}=0, \text { and } \forall k=1, \ldots, m-1, \begin{cases}\hat{\theta}_{k} \in\left[-\hat{w}_{k+1}, \hat{w}_{k+1}\right], & \text { if } \hat{\beta}_{k}=\hat{\beta}_{k+1}, \\ \hat{\theta}_{k}=-\hat{w}_{k+1}, & \text { if } \hat{\beta}_{k}<\hat{\beta}_{k+1}, \\ \hat{\theta}_{k}=\hat{w}_{k+1}, & \text { if } \hat{\beta}_{k}>\hat{\beta}_{k+1} .\end{cases}
$$

Therefore, the proposed algorithm consists in running forwardly through the samples $\left[\mathbf{N}_{k}\right]_{1 \leq k \leq m}$. Using (24), at location $k, \hat{\beta}_{k}$ stays constant where $\left|\hat{\theta}_{k}\right|<\hat{w}_{k+1}$. If this is not possible, it goes back to the last location where a jump can be introduced in $\hat{\beta}$, validates the current segment until this location, starts a new segment, and continues. This algorithm is described precisely in Algorithm 1.

\subsection{Simulated data}

We conduct simulations on 2 examples of intensities. We simulate counting processes with inhomogeneous piecewise intensities $\lambda_{0}$, with 5 and 15 change-points, see Figure 1, with an increasing sample size $n$. In order to assess the performance of the total-variation procedure $\hat{\lambda}$, we use a Monte-Carlo averaged mean integrated squared error (MISE) as a performance measure, given by

$$
\operatorname{MISE}\left(\hat{\lambda}, \lambda_{0}\right)=\mathbb{E} \int_{0}^{1}\left(\hat{\lambda}(t)-\lambda_{0}(t)\right)^{2} d t .
$$

We run 100 Monte-Carlo experiments, for an increasing sample size between $n=500$ and $n=30000$, for each 2 examples. In Figure 2, we plot the MISEs of the weighted and the unweighted total-variation (namely $\hat{w} \equiv 1$ ), for the 2 examples, as a function of the sample size. We observe in Figure 2 that the estimation error is always decaying with the sample size, and that both procedures behave similarly. Differences can be observed 


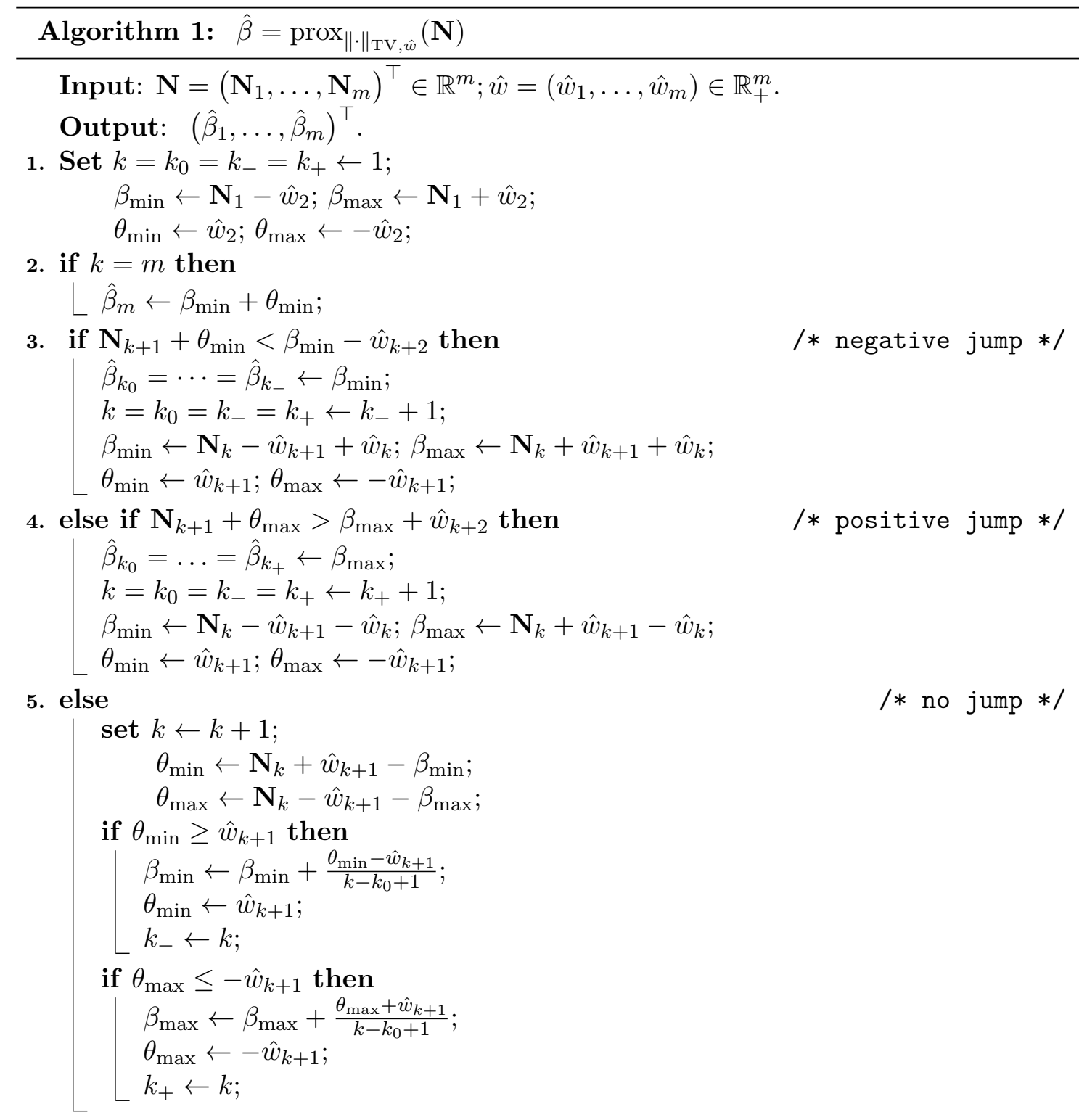

6. if $k<m$ then

$L$ go to 3.;

7. if $\theta_{\min }<0$ then

$$
\begin{aligned}
& \hat{\beta}_{k_{0}}=\cdots=\hat{\beta}_{k_{-}} \leftarrow \beta_{\min } \\
& k=k_{0}=k_{-} \leftarrow k_{-}+1 \\
& \beta_{\min } \leftarrow \mathbf{N}_{k}-\hat{w}_{k+1}+\hat{w}_{k} ; \\
& \theta_{\min } \leftarrow \hat{w}_{k+1} ; \theta_{\max } \leftarrow \mathbf{N}_{k}+\hat{w}_{k}-v_{\max } \\
& \text { go to } 2 .
\end{aligned}
$$

8. else if $\theta_{\max }>0$ then

$$
\begin{aligned}
& \hat{\beta}_{k_{0}}=\cdots=\hat{\beta}_{k_{+}} \leftarrow \beta_{\max } \\
& k=k_{0}=k_{+} \leftarrow k_{+}+1 \\
& \beta_{\max } \leftarrow \mathbf{N}_{k}+\hat{w}_{k+1}-\hat{w}_{k} ; \\
& \theta_{\max } \leftarrow-\hat{w}_{k+1} ; \theta_{\min } \leftarrow \mathbf{N}_{k}-\hat{w}_{k}-\theta_{\min } \\
& \text { go to } \mathbf{2 .}
\end{aligned}
$$

9. else

$$
\hat{\beta}_{k_{0}}=\cdots=\hat{\beta}_{m} \leftarrow \beta_{\min }+\frac{\theta_{\min }}{k-k_{0}+1} ;
$$


below, using a genomics datasets. On each simulated dataset, we perform a 10-fold crossvalidation to select the best constant to use in front of the weights $\hat{w}_{j}$ (both for the weighted and unweighted total-variation). Cross-validation in this context is achieved by choosing uniformly at random a label between 1 and 10 for each point, and by using points with label $k$ in the $k$-th testing fold and removing these points for the $k$-th training fold. The estimated intensity is accordingly corrected, by this amount (as removing uniformly a fraction of points from a counting process biases downwards the intensity by the same fraction).
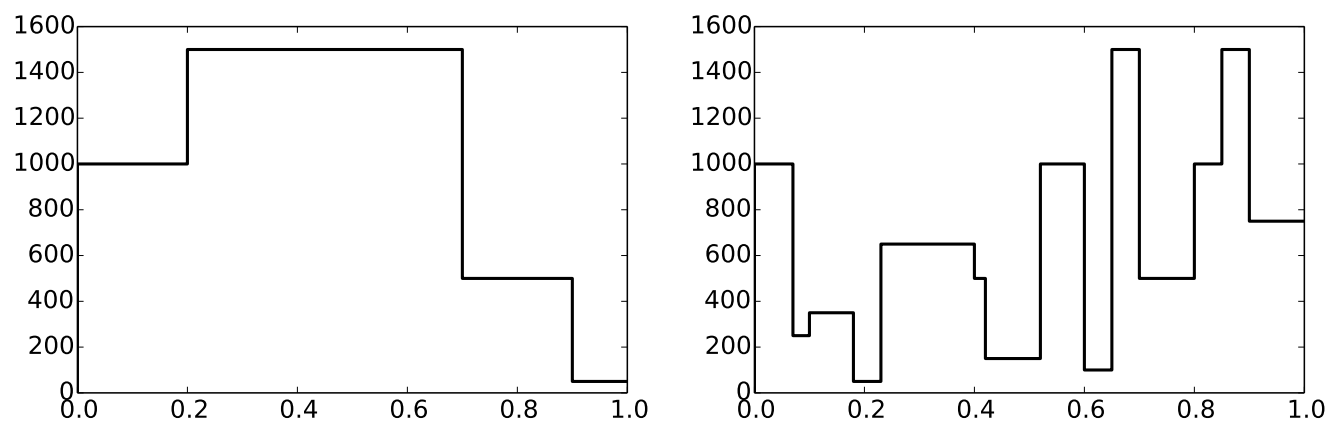

Figure 1: Intensities used for Example 1 (left) and Example 2 (right), respectively with 5 and 15 change-points
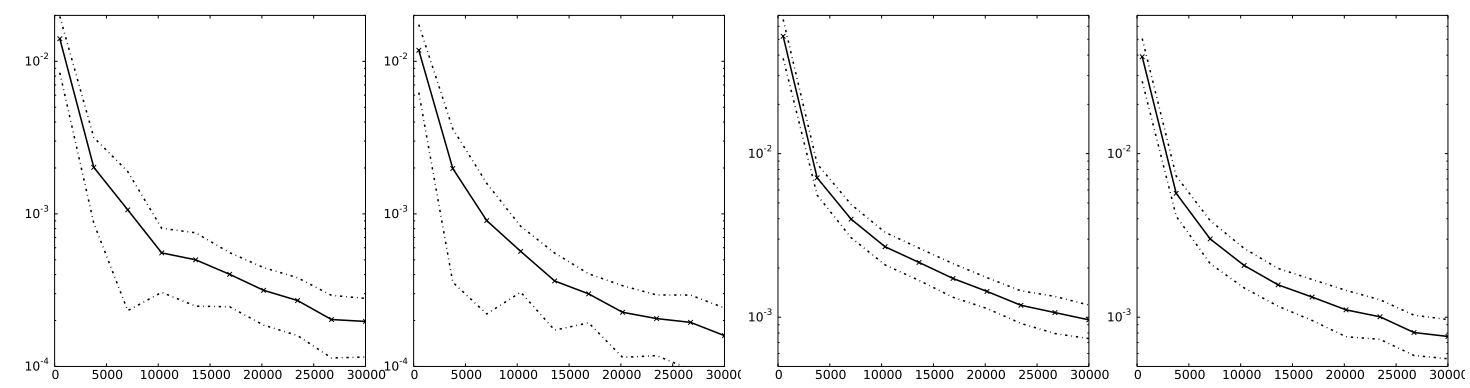

Figure 2: Average MISEs (bold lines) over 100 Monte-Carlo experiments and standard deviations of the MISEs (dashed lines). First: weighted TV for Example 1; Second: nonweighted TV for Example 1; Third: weighted TV for Example 2; Fourth: non-weighted TV for Example 2

\section{$5.3 \quad$ Real data}

Our method is illustrated on NCI-60 tumor and normal cell lines, HCC1954 and BL1954. This dataset was produced and investigated by [14] using the Illumina platform, where the reads are $36 \mathrm{bp}$ long. After cleaning of this data, there are 7.72 million reads for the tumor (HCC1954) and 6.65 million reads for the normal (BL1954) samples respectively. A description of the sampling process for such data is described in Introduction. We show in Figures 3 and 4 both tumor and cell lines data. This data consists of a list of reads number, see Figure 4, where we plot a zoomed sequence of reads. For visualization purposes, we give in Figure 4 the binned counts of reads over 10000 intervals equispaced on the range of reads.

In Figure 5 we plot the best solution of the weighted and unweighted $\left(\hat{w}_{j}=1\right)$ totalvariation estimators on the normal and tumor reads data. For easier visualization we 
Figure 3: A zoom into the sequence of reads for normal (left) and tumor (right) data
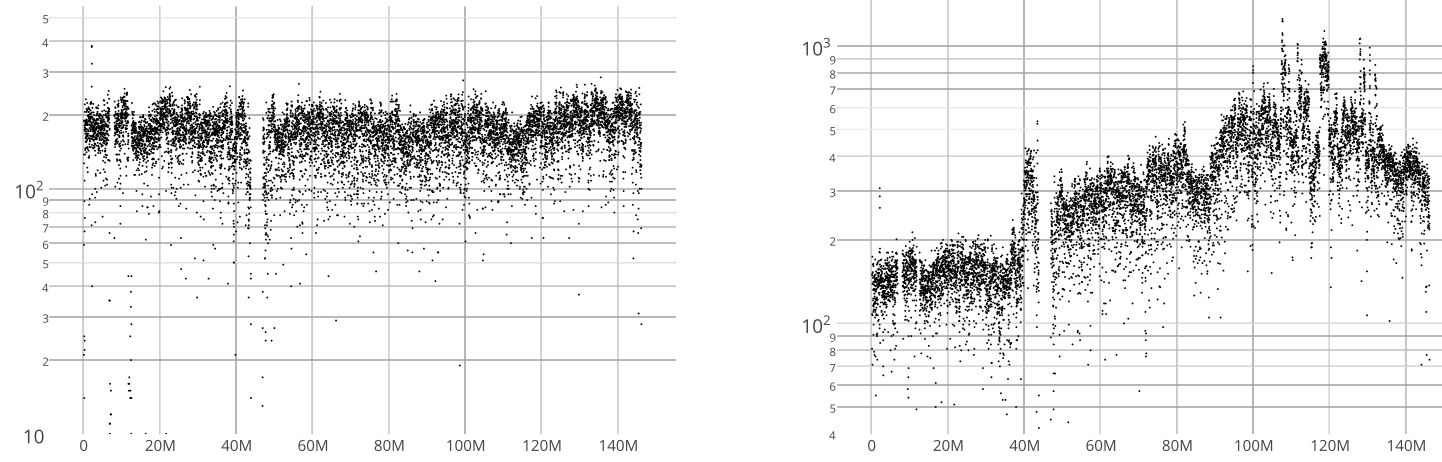

Figure 4: Binned counts of reads (log-scale) of the normal (left) and tumor (right) data
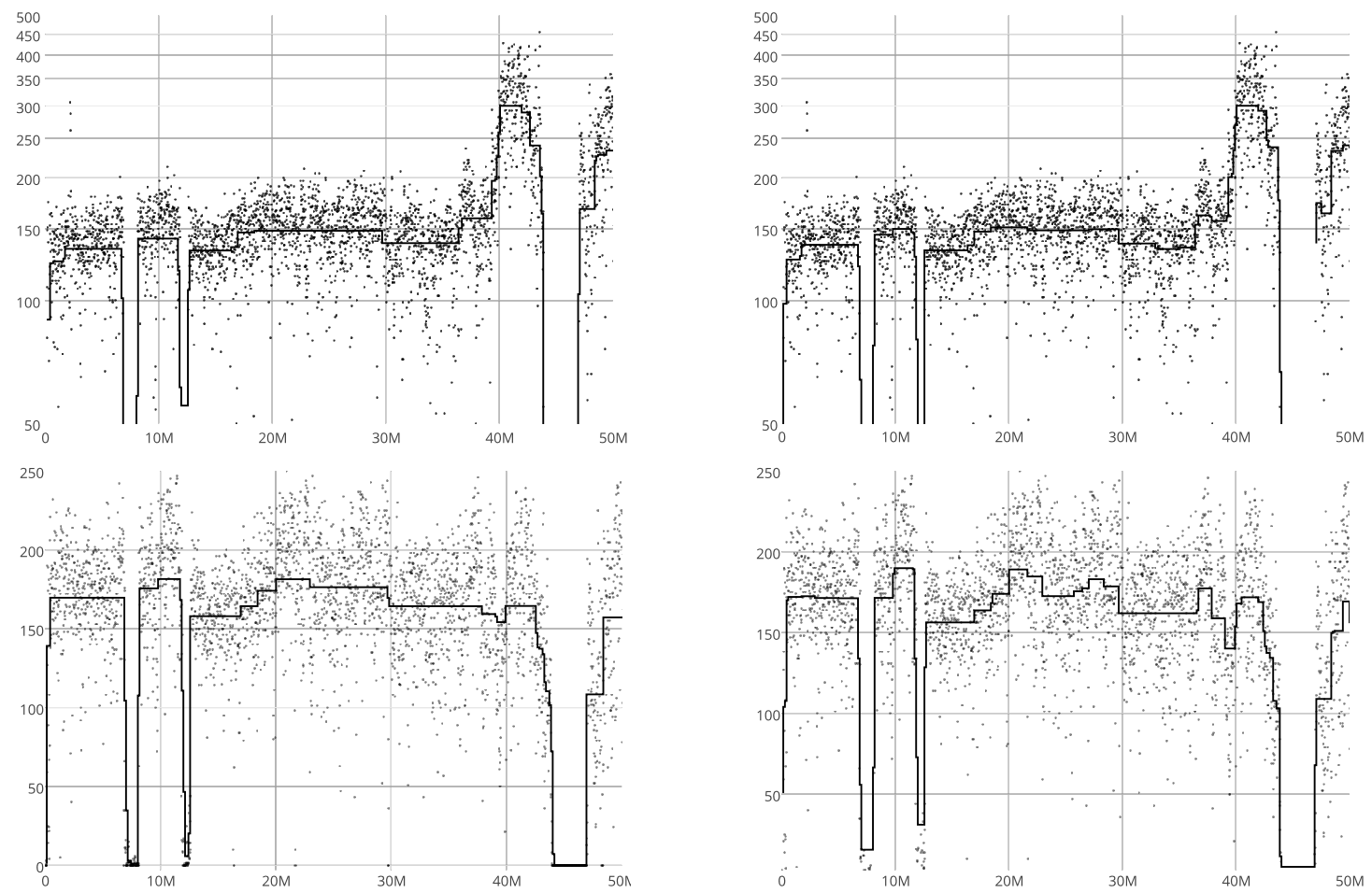

Figure 5: A zoom between reads number 0 and 50M of the weighted (left) and unweighted (right) total-variation estimators applied to the tumor (top) and normal (bottom) data 
plot a zoom of the reads sequence. We perform a 10-fold cross-validation to select the best constant to use in front of the weights $\hat{w}_{j}$ (both for the weighted and unweighted total-variation), as explained above. We observe in this figure that the weighted totalvariation gives sharper results: the piecewise constant intensity is smoother, and the obtained change-points locations seem, at least visually, better. An important fact is that the runtime of Algorithm 1 is extremely fast: a solution is obtained in less than one millisecond, on a modern laptop (implementation is done using python with a $\mathrm{C}$ extension). This is due to the fact that Algorithm 1 is typically linear in the signal size.

\section{Conclusion}

In this work, we prove that convex optimization for the detection of change-points in the intensity of a counting process is a powerful tool. We introduce a data-driven weighted total-variation penalization for this problem, with sharply tuned regularization parameters, and prove two families of theoretical results: oracles inequalities for the prediction error, and consistency in the estimation of change-points. We illustrate numerically our approach via simulations and a genomics dataset application. Future directions for this work are the study of maximum likelihood estimation instead of least-squares, and a multivariate extension of the proposed algorithm.

\section{Proof of Theorems 1 and 2}

Introduce $\mu=\left[\mu_{j}\right]_{1 \leq j \leq m} \in \mathbb{R}^{m}$ given by $\mu_{1}=\beta_{1}$ and $\mu_{j}=\beta_{j}-\beta_{j-1}$ for $j=2, \ldots, m$. Then, we have $\beta=\mathbf{T} \mu$, where $\mathbf{T}$ is the $m \times m$ lower triangular matrix with entries $(\mathbf{T})_{j, k}=0$ if $j<k$ and $(\mathbf{T})_{j, k}=1$ otherwise. Note that $\hat{\beta}=\mathbf{T} \hat{\mu}$, where

$$
\hat{\mu}=\operatorname{argmin}_{\mu \in \mathbb{R}^{m}}\left\{\frac{1}{2}\|\mathbf{N}-\mathbf{T} \mu\|_{2}^{2}+\sum_{j=2}^{m} \hat{w}_{j}\left|\mu_{j}\right|\right\} .
$$

\subsection{Proof of Theorem 1}

This proof follows a standard argument for proving slow oracle inequalities, see for instance [6]. Due to the Doob-Meyer decomposition theorem, we have

$$
R_{n}(\lambda)=\left\|\lambda-\lambda_{0}\right\|^{2}-\left\|\lambda_{0}\right\|^{2}-\int_{0}^{1} \lambda(t) d \bar{M}_{n}(t)
$$

which leads to

$$
\hat{\lambda}=\lambda_{\hat{\beta}}=\operatorname{argmin}_{\beta \in \mathbb{R}_{+}^{m}}\left(\left\|\lambda_{\beta}-\lambda_{0}\right\|^{2}-2 \int_{0}^{1} \lambda_{\beta}(t) d \bar{M}_{n}(t)+\|\beta\|_{\mathrm{TV}, \hat{w}}\right) .
$$

Then, using (6), it implies that

$$
\left\|\hat{\lambda}-\lambda_{0}\right\|^{2} \leq \inf _{\beta}\left\|\lambda_{\beta}-\lambda_{0}\right\|^{2}+\frac{2}{n} \nu_{n}\left(\hat{\lambda}-\lambda_{\beta}\right)+\|\beta\|_{\mathrm{TV}, \hat{w}}-\|\hat{\beta}\|_{\mathrm{TV}, \hat{w}},
$$


where $\nu_{n}(\lambda)=\sum_{i=1}^{n} \int_{0}^{1} \lambda(t) d M_{i}(t)$ is a centered empirical process. Note that

$$
\begin{aligned}
\frac{1}{n} \nu_{n}\left(\hat{\lambda}-\lambda_{\beta}\right) & =\sum_{j=1}^{m}\left(\hat{\beta}_{j, m}-\beta_{j, m}\right) \int_{0}^{1} \lambda_{j, m}(t) d \bar{M}_{n}(t) \\
& =\sum_{j=1}^{m}\left((\mathbf{T} \hat{\mu})_{j, m}-(\mathbf{T} \mu)_{j, m}\right) \int_{0}^{1} \lambda_{j, m}(t) d \bar{M}_{n}(t) \\
& =\sum_{j=1}^{m}\left(\hat{\mu}_{j, m}-\mu_{j, m}\right) \sum_{q=j}^{m} \int_{0}^{1} \lambda_{q, m}(t) d \bar{M}_{n}(t) .
\end{aligned}
$$

Define the event $\Omega_{n}$ by

$$
\Omega_{n}=\bigcap_{j=1}^{m}\left\{\left|\sum_{q=j}^{m} \int_{0}^{1} \lambda_{q, m}(t) d \bar{M}_{n}(t)\right| \leq \frac{\hat{w}_{j}}{2}\right\} .
$$

The probabilistic control of $\Omega_{n}$ is given in Proposition 1 from Section 7 below. It relies on a slight modification of an empirical Bernstein inequality from [18], see also [30]. On $\Omega_{n}$, we have using (28)

$$
\frac{2}{n} \nu_{n}\left(\hat{\lambda}-\lambda_{\beta}\right) \leq \sum_{j=1}^{m} \hat{w}_{j}\left|\hat{\mu}_{j, m}-\mu_{j, m}\right|,
$$

Using (27), we obtain

$$
\begin{aligned}
\left\|\hat{\lambda}-\lambda_{0}\right\|^{2} & \leq\left\|\lambda_{\beta}-\lambda_{0}\right\|^{2}+\sum_{j=1}^{m} \hat{w}_{j}\left|\hat{\mu}_{j, m}-\mu_{j, m}\right|+\sum_{j=1}^{m} \hat{w}_{j}\left(\left|\mu_{j, m}\right|-\left|\hat{\mu}_{j, m}\right|\right) \\
& \leq\left\|\lambda_{\beta}-\lambda_{0}\right\|^{2}+2 \sum_{j=1}^{m} \hat{w}_{j}\left|\mu_{j, m}\right| \\
& =\left\|\lambda_{\beta}-\lambda_{0}\right\|^{2}+2\|\beta\|_{\mathrm{TV}, \hat{w}} .
\end{aligned}
$$

Then, on $\Omega_{n}$, (8) in Theorem 1 holds true . It remains now to control $\mathbb{P}\left(\Omega_{n}^{\complement}\right)$. We have, recalling $\lambda_{j, m}(t)=\sqrt{m} \mathbb{1}_{\left(\frac{j-1}{m}, \frac{j}{m}\right]}(t)$, that

$$
\mathbb{P}\left[\Omega_{n}^{\complement}\right] \leq \sum_{j=1}^{m} \mathbb{P}\left[\left|\sqrt{m} \int_{0}^{1} \mathbb{1}_{\left(\frac{j-1}{m}, 1\right]}(t) d \bar{M}_{n}(t)\right|>\frac{\hat{w}_{j}}{2}\right],
$$

so we need to control the tails of

$$
U_{j}=\int_{0}^{1} \mathbb{1}_{\left(\frac{j-1}{m}, 1\right]}(t) d \bar{M}_{n}(t),
$$

which is the goal of the next proposition.

Proposition 1. For any numerical constants $c_{h}>1, \varepsilon>0$ and $c_{0}>0$ such that $e c_{0}>$ $2(4 / 3+\varepsilon) c_{h}$, the following holds for any $z>0$ :

$$
\mathbb{P}\left[\left|U_{j}\right| \geq c_{1, \varepsilon} \sqrt{\frac{z+\hat{h}_{n, z, j}}{n} \hat{V}_{j}}+c_{3, \varepsilon} \frac{z+1+\hat{h}_{n, z, j}}{n}\right] \leq c e^{-z}
$$

where 


$$
\hat{h}_{n, z, j}=c_{h} \log \log \left(\frac{2 e n \hat{V}_{j}+2 e\left(\frac{4}{3}+\varepsilon\right) z}{e c_{0}(z+1)-2\left(\frac{4}{3}+\varepsilon\right) c_{h}} \vee e\right),
$$

$c_{1, \varepsilon}=2 \sqrt{1+\varepsilon}, c_{3, \varepsilon}=\sqrt{2 \max \left(c_{0}, 2(1+\varepsilon)\left(\frac{4}{3}+\varepsilon\right)\right)}+\frac{1}{3}$, and $c=6+4(\log (1+\varepsilon))^{-c_{h}} \sum_{q \geq 1} q^{-c_{h}}$.

The proof of Proposition 1 is given in Appendix A.1. Choosing $z=x+\log m$, it yields that

$$
\begin{aligned}
\sum_{j=1}^{m} \mathbb{P}\left[\left|U_{j}\right| \geq c_{1, \varepsilon} \sqrt{\frac{x+\log m+\hat{h}_{n, x, j}}{n}} \hat{V}_{j}+c_{3, \varepsilon} \frac{x+\log m+\hat{h}_{n, x, j}+1}{n}\right] \\
\leq\left(6+4(\log (1+\varepsilon))^{-c_{h}} \sum_{q \geq 1} q^{-c_{h}}\right) e^{-x},
\end{aligned}
$$

where

$$
\hat{h}_{n, x, j}=c_{h} \log \log \left(\frac{2 e n \hat{V}_{j}+2 e\left(\frac{4}{3}+\varepsilon\right)(x+\log m)}{e c_{0}(x+\log m+1)-2\left(\frac{4}{3}+\varepsilon\right) c_{h}} \vee e\right) .
$$

Then, the choice of data-driven weights is given by

$$
\hat{w}_{j}=c_{1} \sqrt{\frac{m\left(x+\log m+\hat{h}_{n, x, j}\right) \hat{V}_{j}}{n}}+c_{2} \frac{\sqrt{m}\left(x+1+\log m+\hat{h}_{n, x, j}\right)}{n},
$$

where $c_{1}=2 c_{1, \varepsilon}$ and $c_{2}=2 c_{3, \varepsilon}$ gives $\mathbb{P}\left(\Omega_{n}^{\complement}\right) \leq c e^{-x}$. Finally, to get the numerical constants in Theorem 1, we set $\varepsilon=1, c_{h}=2$, and $c_{0}=28 / 3 e$ in Proposition 1 .

\subsection{Proof of Corollary 1}

We denote by $\lambda_{0, m}$ the projection of $\lambda_{0}$ onto $\Lambda_{m}$, that is $\lambda_{0, m}=\operatorname{argmin}_{\lambda_{\beta} \in \Lambda_{m}}\left\|\lambda_{\beta}-\lambda_{0}\right\|^{2}$. Using Pythagoras' theorem, we have

$$
\left\|\hat{\lambda}-\lambda_{0}\right\|^{2} \leq\left\|\lambda_{0, m}-\lambda_{0}\right\|^{2}+\left\|\hat{\lambda}-\lambda_{0, m}\right\|^{2} .
$$

By the proof of Theorem 1, we obtain

$$
\begin{aligned}
\left\|\hat{\lambda}-\lambda_{0, m}\right\|^{2} & \leq 2\left\|\beta_{0, m}\right\|_{\mathrm{TV}, \hat{w}} \\
& \leq 2\left\|\beta_{0, m}\right\|_{\mathrm{TV}} \max _{1 \leq j \leq m} \hat{w}_{j} .
\end{aligned}
$$

Now, the following approximation lemma comes in handy for the control of the bias term.

Lemma 1. Given Assumption 1, we have

$$
\left\|\lambda_{0, m}-\lambda_{0}\right\|^{2} \leq \frac{2\left(L_{0}-1\right) \Delta_{\beta, \max }^{2}}{m},
$$

where $\Delta_{\beta, \max }=\max _{1 \leq \ell, \ell^{\prime} \leq L_{0}}\left|\beta_{0, \ell}-\beta_{0, \ell^{\prime}}\right|$.

The proof of Lemma 1 is given in Appendix A.2. 


\subsection{Proof of Theorem 2}

Using Pythagoras' identity, we obtain the following decomposition

$$
\left\|\lambda_{\hat{\beta}}-\lambda_{0}\right\|^{2}=\left\|\lambda_{\beta}-\lambda_{0}\right\|^{2}+\left\|\lambda_{\hat{\beta}}-\lambda_{\beta}\right\|^{2} .
$$

In view of the fact that $\left\{\lambda_{j, m}: j=1, \ldots, m\right\}$ is an orthonormal basis of $\Lambda_{m}$, we have

$$
\left\|\lambda_{\hat{\beta}}-\lambda_{\beta}\right\|^{2}=\|\hat{\beta}-\beta\|_{2}^{2},
$$

and by the definition of $\hat{\beta}$, we get

$$
\|\hat{\beta}-\mathbf{N}\|_{2}^{2}+\sum_{j=2}^{m} \hat{w}_{j}\left|\hat{\beta}_{j, m}-\hat{\beta}_{j-1, m}\right| \leq\|\beta-\mathbf{N}\|_{2}^{2}+\sum_{j=2}^{m} \hat{w}_{j}\left|\beta_{j, m}-\beta_{j-1, m}\right| .
$$

Then

$$
\|\hat{\beta}-\beta\|_{2}^{2} \leq \sum_{j=2}^{m} \hat{w}_{j}\left(\left|\beta_{j, m}-\beta_{j-1, m}\right|-\left|\hat{\beta}_{j, m}-\hat{\beta}_{j-1, m}\right|\right)+2 \int_{0}^{1} \sum_{j=2}^{m}\left(\hat{\beta}_{j, m}-\beta_{j, m}\right) \lambda_{j, m}(t) d \bar{M}_{n}(t) .
$$

Assume that $\hat{\beta}$ belongs to a set of dimension at most $L_{\max }$. Let $S=\left\{j: \beta_{j, m} \neq\right.$ $\beta_{j-1, m}$ for $\left.j=2, \ldots, m\right\}$, be the support of the discrete gradient of $\beta$. Using the Cauchy-Schwarz inequality, we have

$$
\begin{aligned}
& \sum_{j=2}^{m} \hat{w}_{j}\left(\left|\beta_{j, m}-\beta_{j-1, m}\right|-\left|\hat{\beta}_{j, m}-\hat{\beta}_{j-1, m}\right|\right) \\
& \quad \leq \sum_{j \in \hat{S} \cup S} \hat{w}_{j}\left(\left|\beta_{j, m}-\hat{\beta}_{j, m}\right|+\left|\beta_{j-1, m}-\hat{\beta}_{j-1, m}\right|\right) \\
& \quad \leq \sum_{j \in \hat{S} \cup S} \hat{w}_{j}\left(\left|\beta_{j, m}-\hat{\beta}_{j, m}\right|\right)+\sum_{j \in \hat{S} \cup S} \hat{w}_{j}\left(\left|\beta_{j-1, m}-\hat{\beta}_{j-1, m}\right|\right) \\
& \quad \leq \sum_{j \in \hat{S} \cup S \cup(\hat{S} \cup S+1)} \hat{w}_{j}\left(\left|\hat{\beta}_{j, m}-\beta_{j, m}\right|\right) \\
& \quad \leq \sqrt{|\hat{S} \cup S \cup(\hat{S}+1) \cup(S+1)|} \\
&\left.\quad \leq \sqrt{2} \sqrt{L_{\max }+2\left(L_{0}-1\right)}\|\hat{\beta}-\beta\|_{2} \hat{\beta}_{j=1, \ldots, m}-\beta_{j, m}\right]_{j \in \hat{S} \cup S \cup(\hat{S}+1) \cup(S+1)} \|_{2} \times \hat{w}_{j \in \hat{S} \cup S \cup(\hat{S}+1) \cup(S+1)} \hat{w}_{j}
\end{aligned}
$$

Hence

$$
\begin{aligned}
& \|\hat{\beta}-\beta\|_{2}^{2} \leq \sqrt{2} \sqrt{L_{\max }+2\left(L_{0}-1\right)}\|\hat{\beta}-\beta\|_{2} \max _{j=1, \ldots, m} \hat{w}_{j} \\
& +2\|\hat{\beta}-\beta\|_{2} \int_{0}^{1} \sum_{j=2}^{m} \frac{\left(\hat{\beta}_{j, m}-\beta_{j, m}\right) \lambda_{j, m}(t)}{\|\hat{\beta}-\beta\|_{2}} d \bar{M}_{n}(t) .
\end{aligned}
$$

Now, define the functional $G$ for all $\lambda_{\beta} \in \Lambda_{m}$ in the following way:

$$
G\left(\lambda_{\beta}\right)=\int_{0}^{1} \frac{\lambda_{\beta}(t)}{\left\|\lambda_{\beta}\right\|} d \bar{M}_{n}(t)
$$


Therefore, we obtain

$$
\|\hat{\beta}-\beta\|_{2}^{2} \leq \sqrt{2} \sqrt{L_{\max }+2\left(L_{0}-1\right)}\|\hat{\beta}-\beta\|_{2} \max _{j=1, \ldots, m} \hat{w}_{j}+2\|\hat{\beta}-\beta\|_{2} G(\hat{\beta}-\beta) .
$$

Let

$$
\mathcal{V}=\bigcup_{L=1}^{L_{\max }} V_{L}=\bigcup_{L=1}^{L_{\max }} \bigcup_{J \subset\{1, \ldots, m-1\},|J|=L} V_{L, J},
$$

where $\left\{V_{L}: L=1, \ldots, L_{\max }\right\}$ is the collection of the spaces to which $\hat{\beta}$ may belong and $V_{L, J}$ denotes a space of dimension $L$ containing signals with a support $J$.

It follows that,

$$
\|\hat{\beta}-\beta\|_{2} \leq \sqrt{2} \sqrt{L_{\max }+2\left(L_{0}-1\right)} \max _{j=1, \ldots, m} \hat{w}_{j}+2 \sup _{\lambda \in \mathcal{V},\|\lambda\|=1} G(\lambda) .
$$

Then by Proposition 4 in [16], we have for any $z>0$

$$
\mathbb{P}\left[\sup _{\lambda \in V_{L, J},\|\lambda\|=1} G(\lambda) \geq \kappa\left(\sqrt{\frac{\left\|\lambda_{0}\right\|_{\infty}(L+z)}{n}}+\frac{2 \sqrt{m}(L+z)}{\sqrt{L} n}\right)\right] \leq e^{-z},
$$

where $\kappa=11.8$. Then

$$
\begin{aligned}
& \quad \sum_{\substack{L=1, \ldots, L_{\max } \\
J \subset\{1, \ldots, m-1\},|J|=L}} \mathbb{P}\left[\sup _{\lambda \in V_{L, J},\|\lambda\|=1} G(\lambda) \geq \kappa\left(\sqrt{\frac{\left\|\lambda_{0}\right\|_{\infty}(L+z)}{n}}+\frac{2 \sqrt{m}(L+z)}{\sqrt{L} n}\right)\right] \\
& \leq \sum_{\substack{L=1, \ldots, L_{\max } \\
J \subset\{1, \ldots, m-1\},|J|=L}} e^{-z} \\
& \leq L_{\max } m^{L_{\max }} e^{-z} .
\end{aligned}
$$

Choosing $z=x+L_{\max } \log m$ for $x>0$, leads to

$$
\begin{aligned}
& \sum_{\substack{L=1, \ldots, L_{\max } \\
J \subset\{1, \ldots, m-1\},|J|=L}} \mathbb{P}\left[\sup _{\lambda \in V_{L, J},\|\lambda\|=1} G(\lambda) \geq \kappa\left(\sqrt{\frac{\left\|\lambda_{0}\right\|_{\infty}\left(L+x+L_{\max } \log m\right)}{n}}\right.\right. \\
& \left.\left.\quad+\frac{2 \sqrt{m}\left(L+x+L_{\max } \log m\right)}{\sqrt{L} n}\right)\right] \\
& \leq L_{\max } e^{-x} .
\end{aligned}
$$

Plugging this in inequality (29), we obtain for any $x>0$ and with probability larger than $1-L_{\max } e^{-x}$

$$
\begin{aligned}
\|\hat{\beta}-\beta\|_{2} \leq & \sqrt{2} \sqrt{L_{\max }+2\left(L_{0}-1\right)} \max _{j=1, \ldots, m} \hat{w}_{j} \\
& +2 \kappa \sqrt{\frac{\left\|\lambda_{0}\right\|_{\infty}\left(x+L_{\max }(1+\log m)\right)}{n}} \\
& +4 \kappa \frac{\sqrt{m}\left(x+L_{\max }(1+\log m)\right)}{n},
\end{aligned}
$$

and the result follows by using the inequality $(a+b+c)^{2} \leq 3\left(a^{2}+b^{2}+c^{2}\right)$, for all $a, b, c \in \mathbb{R}$. 


\section{Proof of Theorem 3}

Let us give first the overall structure of the proof, which is inspired from [21]. In this proof, we repeatedly use the KKT optimality conditions of the optimization problem (25), given by Lemma 2 below. We use also repeatedly deviation arguments of the data-driven weights $\hat{w}_{j}$ and a control of the martingale noise, which are provided by Lemma 3 below. We prove consistency of $\hat{\tau}_{\ell}=\frac{\hat{j}_{\ell}}{m}$, which is an estimator of the right-hand side boundary $\frac{j_{\ell}}{m}$ of the interval $I_{j_{\ell}, m}=\left(\frac{j_{\ell}-1}{m}, \frac{j_{\ell}}{m}\right]$, by showing that $\mathbb{P}\left[A_{n, \ell}\right] \rightarrow 0$ as $n \rightarrow \infty$, where $A_{n, \ell}:=\left\{\left|\hat{j}_{\ell}-j_{\ell}\right|>\frac{m \varepsilon_{n}}{2}\right\}$, for all $\ell \in\left\{1, \ldots, L_{0}-1\right\}$. We treat separately two cases depending on the positions of $j_{\ell}$ and $\hat{j}_{\ell}$. In Case I, we consider $\hat{j}_{\ell}<j_{\ell}$, see Section 8.1 and Figure 6. In Case II., we consider $\hat{j}_{\ell}>j_{\ell}$, , see Appendix B and Figure 7. We decompose even further, using the quantity $\Delta_{j, \text { min }}$ (see Section 4 ), defining the set $C_{n}=\left\{\max _{1 \leq \ell \leq L_{0}-1}\left|\hat{j}_{\ell}-j_{\ell}\right|<\frac{\Delta_{j, \min }}{2}\right\}$. We prove that $\mathbb{P}\left[A_{n, \ell} \cap C_{n}\right] \rightarrow 0$ and $\mathbb{P}\left[A_{n, \ell} \cap C_{n}^{\complement}\right] \rightarrow 0$ as $n \rightarrow \infty$ for Case I in Sections 8.1.1, 8.1.2, and for Case II in Appendices B.1, B.2.

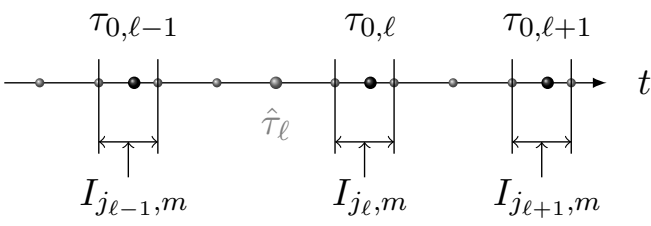

Figure 6: Case I. $\hat{j}_{\ell}<j_{\ell}$

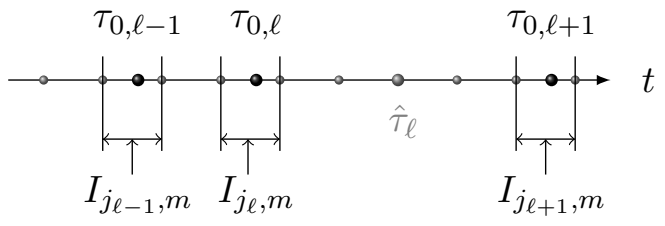

Figure 7: Case II. $\hat{j}_{\ell}>j_{\ell}$

Lemma 2. Consider the total-variation penalized problems in (21) and(25). Let $\hat{\beta}=$ $\left[\hat{\beta}_{j, m}\right]_{1 \leq j \leq m}$ and $\hat{\mu}=\left[\hat{\mu}_{j, m}\right]_{1 \leq j \leq m}$ denote the respective solutions. Then, the latter vectors and the approximate change-points sequence estimators $\hat{j}_{1}, \ldots, \hat{j}_{|\hat{S}|}$ satisfy for all $r=1, \ldots,|\hat{S}|$,

$$
\sum_{j=\hat{j}_{r}}^{m} \beta_{0, j, m}-\sum_{j=\hat{j}_{r}}^{m} \hat{\beta}_{j, m}+\sqrt{m} \sum_{j=\hat{j}_{r}}^{m} \bar{M}_{n}\left(I_{j, m}\right)=\hat{w}_{\hat{j}_{r}} \operatorname{sign}\left(\hat{\mu}_{\hat{j}_{r}, m}\right),
$$

and for all $j \in\{1, \ldots, m\}$,

$$
\left|\sum_{q=j}^{m} \beta_{0, q, m}-\sum_{q=j}^{m} \hat{\beta}_{q, m}+\sqrt{m} \sum_{q=j}^{m} \bar{M}_{n}\left(I_{q, m}\right)\right| \leq \hat{w}_{j},
$$

using the convention $\operatorname{sign}\left(\hat{\mu}_{\hat{j}_{r}, m}\right)=+1$, if $\hat{\mu}_{\hat{j}_{r}, m}>0$ and -1 otherwise. The vectors $\hat{\beta}$ and $\beta_{0, m}=\left[\beta_{0, j, m}\right]_{1 \leq j \leq m}$ have the following additional properties

$$
\begin{cases}\hat{\beta}_{q, m}=\hat{\beta}_{\hat{j}_{r}-1, m}, & \text { if } \hat{j}_{r-1}+1 \leq q \leq \hat{j}_{r}, \text { for } r=1, \ldots, \hat{L}, \\ \beta_{0, q, m}=\beta_{0, j_{\ell}-1, m}, & \text { if } j_{\ell-1}+1 \leq q \leq j_{\ell}-1, \text { for } \ell=1, \ldots, L_{0}-1 .\end{cases}
$$

The proof of Lemma 2 is given in Appendix A.3. Let us now state a lemma which allows us to control the martingale noise term.

Lemma 3. Given two integers $a$ and $b$, such that $1 \leq a<b \leq m$, let $\bar{M}_{n}(a ; b):=$ $\sum_{q=a}^{b} \bar{M}_{n}\left(I_{q, m}\right)$. Then, for all $z>0$ we have

$$
\mathbb{P}\left[\left|\bar{M}_{n}(a ; b)\right| \geq z\right] \leq 2 \exp \left(-\frac{n z^{2}}{2 \int_{\mathbb{1}_{\left(\frac{a-1}{m}, \frac{b}{m}\right]}} \lambda_{0}(t) d t+\frac{2}{3} z}\right),
$$


and for all $\xi>0$, the data driven weight $\hat{w}_{a}$ satisfies

$$
\mathbb{P}\left[\hat{w}_{a}^{2} \geq \frac{m \log m}{n}\left(\xi-\int_{\mathbb{1}_{\left(\frac{a-1}{m}, 1\right]}} \lambda_{0}(t) d t\right)\right] \leq 2 \exp \left(-\frac{n \xi^{2}}{2 \int_{\mathbb{1}_{\left(\frac{a-1}{m}, 1\right]}} \lambda_{0}(t) d t+\frac{2}{3} \xi}\right),
$$

where $\int_{I} \lambda_{0}(t) d t=\mathbb{E}[\bar{N}(I)]$ for any $I \subset[0,1]$.

The proof of Lemma 3 is given in Appendix A.4. Let us now prove Theorem 3. Recall that the sequence $\left(\varepsilon_{n}\right)_{n}$ satisfies $m \varepsilon_{n} \geq 6$, for all $n \geq 1$. An application of the triangle inequality entails that,

$\mathbb{P}\left[\max _{1 \leq \ell \leq L_{0}-1}\left|\tau_{0, \ell}-\hat{\tau}_{\ell}\right|>\varepsilon_{n}\right] \leq \mathbb{P}\left[\max _{1 \leq \ell \leq L_{0}-1}\left|\tau_{0, \ell}-\frac{j_{\ell}}{m}\right|>\frac{\varepsilon_{n}}{2}\right]+\mathbb{P}\left[\max _{1 \leq \ell \leq L_{0}-1}\left|\frac{j_{\ell}}{m}-\hat{\tau}_{\ell}\right|>\frac{\varepsilon_{n}}{2}\right]$.

Moreover, the true change-point $\tau_{0, \ell}$ verifies (14) which implies that

$$
\mathbb{P}\left[\max _{1 \leq \ell \leq L_{0}-1}\left|\tau_{0, \ell}-\hat{\tau}_{\ell}\right|>\varepsilon_{n}\right] \leq \mathbb{P}\left[\max _{1 \leq \ell \leq L_{0}-1}\left|j_{\ell}-\hat{j}_{\ell}\right|>\frac{m \varepsilon_{n}}{2}\right] .
$$

Due to

$$
\mathbb{P}\left[\max _{1 \leq \ell \leq L_{0}-1}\left|\hat{j}_{\ell}-j_{\ell}\right|>\frac{m \varepsilon_{n}}{2}\right] \leq \sum_{\ell=1}^{L_{0}-1} \mathbb{P}\left[\left|\hat{j}_{\ell}-j_{\ell}\right|>\frac{m \varepsilon_{n}}{2}\right]
$$

it suffices to prove that for all $\ell=1, \ldots, L_{0}-1, \mathbb{P}\left[A_{n, \ell}\right] \rightarrow 0$, as $n$ tending to infinity.

\subsection{Case I}

Due to the fact that $m \varepsilon_{n} \geq 6$ for all $n \geq 1$, it follows that the event $\left\{\hat{j}_{\ell}<j_{\ell}-2\right\}$ a.s.

8.1.1 Step I.1. Prove: $\mathbb{P}\left[A_{n, \ell} \cap C_{n}\right] \rightarrow 0$, as $n \rightarrow \infty$.

By the definition of $C_{n}$, we have

$$
j_{\ell-1}<\hat{j}_{\ell}<j_{\ell+1}, \text { for all } \ell=1, \ldots, L_{0}-1 .
$$

Applying (31) in Lemma 2 with $j=j_{\ell}$ and $j=\hat{j}_{\ell}+1$, we obtain

$$
-\left(\hat{w}_{j_{\ell}}+\hat{w}_{\hat{j}_{\ell}+1}\right) \leq \sum_{q=\hat{j}_{\ell}+1}^{j_{\ell}-1} \mathbf{N}_{q}-\sum_{q=\hat{j}_{\ell}+1}^{j_{\ell}-1} \hat{\beta}_{q, m} \leq \hat{w}_{j_{\ell}}+\hat{w}_{\hat{j}_{\ell}+1} .
$$

Put $\hat{w}_{a, b}:=\hat{w}_{a}+\hat{w}_{b}$, for any two integers $a$ and $b$. Thus

$$
\left|\sum_{q=\hat{j}_{\ell}+1}^{j_{\ell}-1} \beta_{0, q, m}-\hat{\beta}_{q, m}+\sqrt{m} \bar{M}_{n}\left(I_{q, m}\right)\right| \leq \hat{w}_{\hat{j}_{\ell}+1, j_{\ell}} .
$$

Using the property of the vector $\hat{\beta}$ in Lemma 2 , we get

$$
\left|\left(j_{\ell}-\hat{j}_{\ell}-2\right)\left(\beta_{0, j_{\ell}-1, m}-\hat{\beta}_{\hat{j}_{\ell+1}-1, m}\right)+\sqrt{m} \bar{M}_{n}\left(\hat{j}_{\ell}+1 ; j_{\ell}-1\right)\right| \leq \hat{w}_{\hat{j}_{\ell}+1, j_{\ell}} .
$$


Therefore, on $C_{n} \cap\left\{\hat{j}_{\ell}<j_{\ell}-2\right\}$, we have

$$
\begin{aligned}
& \mid\left(\hat{j}_{\ell}-j_{\ell}-2\right)\left(\hat{\beta}_{\hat{j}_{\ell+1}-1, m}-\beta_{0, j_{\ell+1}-1, m}\right) \\
& +\left(\hat{j}_{\ell}-j_{\ell}-2\right)\left(\beta_{0, j_{\ell+1}-1, m}-\beta_{0, j_{\ell}-1, m}\right) \\
& \quad+\sqrt{m} \bar{M}_{n}\left(\hat{j}_{\ell}+1 ; j_{\ell}-1\right) \mid \leq \hat{w}_{\hat{j}_{\ell}+1, j_{\ell}} .
\end{aligned}
$$

Defining the event

$$
\begin{aligned}
& C_{n, \ell}=\left\{\mid\left(\hat{j}_{\ell}-j_{\ell}-2\right)\left(\hat{\beta}_{\hat{j}_{\ell+1}-1, m}-\beta_{0, j_{\ell+1}-1, m}\right)\right. \\
& +\left(\hat{j}_{\ell}-j_{\ell}-2\right)\left(\beta_{0, j_{\ell+1}-1, m}-\beta_{0, j_{\ell}-1, m}\right) \\
& \left.\quad+\sqrt{m} \bar{M}_{n}\left(\hat{j}_{\ell}+1 ; j_{\ell}-1\right)+\mid \leq \hat{w}_{\hat{j}_{\ell}+1, j_{\ell}}\right\},
\end{aligned}
$$

We observe that $C_{n, \ell}$ occurs with probability one. In addition, we remark that for all $n \geq 1, m \varepsilon_{n} \geq 6$ entails $\frac{m \varepsilon_{n}}{2}-2 \geq \frac{m \varepsilon_{n}}{6}$. Then

$$
\left\{\left|\hat{j}_{\ell}-j_{\ell}\right|>\frac{m \varepsilon_{n}}{2}\right\} \subset\left\{\left|\hat{j}_{\ell}-j_{\ell}-2\right|>\frac{m \varepsilon_{n}}{2}-2\right\} \subset\left\{\left|\hat{j}_{\ell}-j_{\ell}-2\right| \geq \frac{m \varepsilon_{n}}{6}\right\}
$$

Therefore

$$
\begin{aligned}
& \mathbb{P}\left[A_{n, \ell} \cap C_{n} \cap C_{n, \ell}\right] \\
& \leq \mathbb{P}\left[\left\{\frac{\hat{w}_{\hat{j}_{\ell}+1, j_{\ell}}}{\left|\hat{j}_{\ell}-j_{\ell}-2\right|} \geq \frac{\left|\beta_{0, j_{\ell+1}-1, m}-\beta_{0, j_{\ell}-1, m}\right|}{3}\right\} \cap\left\{\hat{j}_{\ell}<j_{\ell}-2\right\}\right] \\
&+\mathbb{P}\left[\left\{\left|\hat{\beta}_{\hat{j}_{\ell+1}-1, m}-\beta_{0, j_{\ell+1}-1, m}\right| \geq \frac{\left|\beta_{0, j_{\ell+1}-1, m}-\beta_{0, j_{\ell}-1, m}\right|}{3}\right\} \cap C_{n}\right] \\
&+\mathbb{P}\left[\left\{\left|\frac{\sqrt{m} \bar{M}_{n}\left(\hat{j}_{\ell}+1 ; j_{\ell}-1\right)}{\hat{j}_{\ell}-j_{\ell}-2}\right| \geq \frac{\left|\beta_{0, j_{\ell+1}-1, m}-\beta_{0, j_{\ell}-1, m}\right|}{3}\right\}\right] \\
&:= \mathbb{P}\left[A_{n, \ell, 1}\right]+\mathbb{P}\left[A_{n, \ell, 2}\right]+\mathbb{P}\left[A_{n, \ell, 3}\right] .
\end{aligned}
$$

Moreover, we have

$$
\begin{aligned}
\mathbb{P}\left[A_{n, \ell, 1}\right] & \leq \mathbb{P}\left[\hat{w}_{\hat{j}_{\ell}+1, j_{\ell}} \geq \frac{m \varepsilon_{n} \Delta_{\beta, \min }}{18}\right] \\
& \leq \mathbb{P}\left[\hat{w}_{\hat{j}_{\ell+1}} \geq \frac{m \varepsilon_{n} \Delta_{\beta, \min }}{36}\right] \\
& \leq \mathbb{P}\left[\hat{w}_{j_{\ell-1}+1}^{2} \geq \frac{m^{2} \varepsilon_{n}^{2} \Delta_{\beta, \min }^{2}}{36^{2}}\right] .
\end{aligned}
$$

By (16) in Assumption 4, and (34) in Lemma 3 with $\xi=\frac{n m \varepsilon_{n}^{2} \Delta_{\beta, \min }^{2}}{36^{2} \log m}+\mathbb{E}\left[\bar{N}_{n}\left(\left(\frac{j_{\ell-1}}{m}, 1\right]\right)\right]$, it follows that

$$
\mathbb{P}\left[A_{n, \ell, 1}\right] \leq 2 \exp \left(-\frac{n \xi^{2}}{\left.2 \mathbb{E}\left[\bar{N}_{n}\left(\left(\frac{j \ell-1}{m}, 1\right]\right)\right)\right]+\frac{2}{3} \xi}\right) \rightarrow 0
$$


as $n \rightarrow \infty$. Next, consider the event

$$
\begin{aligned}
A_{n, \ell, 3} & =\left\{\left|\frac{\sqrt{m} \bar{M}_{n}\left(\hat{j}_{\ell}+1 ; j_{\ell}-1\right)}{\hat{j}_{\ell}-j_{\ell}-2}\right| \geq \frac{\left|\beta_{0, j_{\ell+1}-1, m}-\beta_{0, j_{\ell}-1, m}\right|}{3}\right\} \\
& =\left\{\left|\bar{M}_{n}\left(\hat{j}_{\ell}+1 ; j_{\ell}-1\right)\right| \geq\left|\hat{j}_{\ell}-j_{\ell}-2\right| \frac{\left|\beta_{0, j_{\ell+1}-1, m}-\beta_{0, j_{\ell}-1, m}\right|}{3 \sqrt{m}}\right\} \\
& \subset\left\{\left|\bar{M}_{n}\left(\hat{j}_{\ell}+1 ; j_{\ell}-1\right)\right| \geq \frac{m \varepsilon_{n} \Delta_{\beta, \min }}{18 \sqrt{m}}\right\} \bigcap \bigcup_{q=j_{\ell-1}+1}^{j_{\ell}-3}\left\{\hat{j}_{\ell}=q\right\} \\
& \subset \bigcup_{q=j_{\ell-1}+2}^{j_{\ell}-2}\left\{\left|\bar{M}_{n}\left(q ; j_{\ell}-1\right)\right| \geq \frac{m \varepsilon_{n} \Delta_{\beta, \min }}{18 \sqrt{m}}\right\} .
\end{aligned}
$$

Put $\varphi_{n}=\frac{\sqrt{m} \varepsilon_{n} \Delta_{\beta, \min }}{18}$. By (33) in Lemma 3, we have

$$
\begin{aligned}
\mathbb{P}\left[A_{n, \ell, 3}\right] & \leq 2 \sum_{q=j_{\ell-1}+2}^{j_{\ell}-2} \exp \left(-\frac{n \varphi_{n}^{2}}{2 \mathbb{E}\left[\bar{N}_{n}\left(\left(\frac{q-1}{m}, \frac{j_{\ell}-1}{m}\right]\right)\right]+\frac{2}{3} \varphi_{n}}\right) \\
& \leq 2\left(j_{\ell}-j_{\ell-1}-3\right) \exp \left(-\frac{n \varphi_{n}^{2}}{2 \mathbb{E}\left[\bar{N}_{n}\left(\left(\frac{j_{\ell-1}+1}{m}, \frac{j_{\ell}-1}{m}\right]\right)\right]+\frac{2}{3} \varphi_{n}}\right) \\
& \leq 2 \exp \left(-\frac{n \varphi_{n}^{2}}{2 \mathbb{E}\left[\bar{N}_{n}\left(\left(\frac{j_{\ell-1}+1}{m}, \frac{j_{\ell}-1}{m}\right]\right)\right]+\frac{2}{3} \varphi_{n}}+\log m\right) .
\end{aligned}
$$

By (16) in Assumption 4 , it implies that $\mathbb{P}\left[A_{n, \ell, 3}\right]$ goes to zero as $n \rightarrow \infty$. We now control $\mathbb{P}\left[A_{n, \ell, 2}\right]$. Using Lemma 2 with $j=\left\lceil\frac{j_{\ell}+j_{\ell+1}}{2}\right\rceil$ and with $j=j_{\ell}+1$, and using the triangle inequality, it follows that

$$
\left|\sum_{q=j_{\ell}+1}^{\left\lceil\frac{j_{\ell}+j_{\ell+1}}{2}\right\rceil-1} \mathbf{N}_{q}-\sum_{q=j_{\ell}+1}^{\left\lceil\frac{j_{\ell}+j_{\ell+1}}{2}\right\rceil-1} \hat{\beta}_{q, m}\right| \leq \hat{w}_{j_{\ell}+1,\left\lceil\frac{j_{\ell}+j_{\ell+1}}{2}\right\rceil} .
$$

Furthermore, on the event $C_{n} \cap\left\{\hat{j}_{\ell}<j_{\ell}-2\right\}$, the following inequalities

$$
\hat{j}_{\ell}<j_{\ell} \leq q \leq\left\lceil\frac{j_{\ell}+j_{\ell+1}}{2}\right\rceil-1 \leq j_{\ell+1}-1,
$$

hold true. Moreover, we note that $\hat{\beta}_{q, m}=\hat{\beta}_{\hat{j}_{\ell+1}-1, m}$ if $j_{\ell} \leq q \leq\left\lceil\frac{j_{\ell}+j_{\ell+1}}{2}\right\rceil-1 \leq \hat{j}_{\ell+1}-1$. Consequently, we have

$$
\left|\left(j_{\ell+1}-j_{\ell}-2\right) \frac{\left(\beta_{0, j_{\ell+1}-1, m}-\hat{\beta}_{\hat{j}_{\ell+1}-1, m}\right)}{2}+\sqrt{m} \bar{M}_{n}\left(j_{\ell}+1 ;\left\lceil\frac{j_{\ell}+j_{\ell+1}}{2}\right\rceil-1\right)\right| \leq \hat{w}_{j_{\ell}+1,\left\lceil\frac{j_{\ell}+j_{\ell+1}}{2}\right\rceil},
$$

which implies that

$$
\left(j_{\ell+1}-j_{\ell}-2\right) \frac{\left|\hat{\beta}_{\hat{j}_{\ell+1}-1, m}-\beta_{0, j_{\ell+1}-1, m}\right|}{2} \leq \hat{w}_{j_{\ell}+1,\left\lceil\frac{j_{\ell}+j_{\ell+1}}{2}\right\rceil}+\left|\sqrt{m} \bar{M}_{n}\left(j_{\ell}+1 ;\left\lceil\frac{j_{\ell}+j_{\ell+1}}{2}\right\rceil-1\right)\right| .
$$


Therefore, we may upper bound $\mathbb{P}\left[A_{n, \ell, 2}\right]$ as follows

$$
\begin{aligned}
& \mathbb{P}\left[A_{n, \ell, 2}\right] \\
& =\mathbb{P}\left[\left\{\left|\hat{\beta}_{\hat{j}_{\ell+1}-1, m}-\beta_{0, j_{\ell+1}-1, m}\right| \geq \frac{\left|\beta_{0, j_{\ell+1}-1, m}-\beta_{0, j_{\ell}-1, m}\right|}{3}\right\} \cap C_{n}\right] \\
& =\mathbb{P}\left[\left\{\left(j_{\ell+1}-j_{\ell}-2\right) \frac{\left|\hat{\beta}_{\hat{j}_{\ell+1}, m}-\beta_{0, j_{\ell+1}-1, m}\right|}{2}\right.\right. \\
& \left.\left.\geq\left(j_{\ell+1}-j_{\ell}-2\right) \frac{\left|\beta_{0, j_{\ell+1}-1, m}-\beta_{0, j_{\ell}-1, m}\right|}{6}\right\} \cap C_{n}\right] \\
& \leq \mathbb{P}\left[\left\{\hat{w}_{j_{\ell}+1,\left\lceil\frac{j_{\ell}+j_{\ell+1}}{2}\right\rceil}+\left|\sqrt{m} \bar{M}_{n}\left(j_{\ell}+1 ;\left\lceil\frac{j_{\ell}+j_{\ell+1}}{2}\right\rceil-1\right)\right|\right.\right. \\
& \left.\left.\geq\left(j_{\ell+1}-j_{\ell}-2\right) \frac{\left|\beta_{0, j_{\ell+1}-1, m}-\beta_{0, j_{\ell}-1, m}\right|}{6}\right\} \cap C_{n}\right] \\
& \leq \mathbb{P}\left[\hat{w}_{j_{\ell}+1,\left\lceil\frac{j_{\ell}+j_{\ell+1}}{2}\right\rceil} \geq\left(j_{\ell+1}-j_{\ell}-2\right) \frac{\left|\beta_{0, j_{\ell+1}-1, m}-\beta_{0, j_{\ell}-1, m}\right|}{12}\right] \\
& +\mathbb{P}\left[\left|\sqrt{m} \bar{M}_{n}\left(j_{\ell}+1 ;\left\lceil\frac{j_{\ell}+j_{\ell+1}}{2}\right\rceil-1\right)\right| \geq\left(j_{\ell+1}-j_{\ell}-2\right) \frac{\left|\beta_{0, j_{\ell+1}-1, m}-\beta_{0, j_{\ell}-1, m}\right|}{12}\right] \\
& \leq \mathbb{P}\left[\hat{w}_{j_{\ell}+1,\left\lceil\frac{j_{\ell}+j_{\ell+1}}{2}\right\rceil} \geq \frac{\left(\Delta_{j, \min }-2\right) \Delta_{\beta, \min }}{12}\right] \\
& +\mathbb{P}\left[\left|\bar{M}_{n}\left(j_{\ell}+1 ;\left\lceil\frac{j_{\ell}+j_{\ell+1}}{2}\right\rceil-1\right)\right| \geq \frac{\left(\Delta_{j, \min }-2\right) \Delta_{\beta, \min }}{12 \sqrt{m}}\right] .
\end{aligned}
$$

On the other hand, it is easy to see that (13) in Assumption 3 yields that $\Delta_{j, \min }-2 \geq$ $\frac{\Delta_{j, \min }}{2}-2 \geq \frac{\Delta_{j, \min }}{6}$. Thus

$$
\begin{aligned}
\mathbb{P}\left[A_{n, \ell, 2}\right] \leq & \mathbb{P}\left[\hat{w}_{j_{\ell}+1,\left\lceil\frac{j_{\ell}+j_{\ell+1}}{2}\right\rceil} \geq \frac{\Delta_{j, \min } \Delta_{\beta, \min }}{72}\right] \\
& +\mathbb{P}\left[\left|\bar{M}_{n}\left(j_{\ell}+1 ;\left\lceil\frac{j_{\ell}+j_{\ell+1}}{2}\right\rceil-1\right)\right| \geq \frac{\Delta_{j, \min } \Delta_{\beta, \text { min }}}{72 \sqrt{m}}\right] \\
& :=\alpha_{n, \ell, 2}^{(1)}+\alpha_{n, \ell, 2}^{(2)} .
\end{aligned}
$$

Using the property of the data-driven weights, we remark that

$$
\alpha_{n, \ell, 2}^{(1)} \leq \mathbb{P}\left[\hat{w}_{j_{\ell}+1}^{2} \geq \frac{\Delta_{j, \min }^{2} \Delta_{\beta, \min }^{2}}{144^{2}}\right] .
$$

By (17) in Assumption 4, and (34) in Lemma 3 with $\xi=\frac{n \Delta_{j, \min }^{2} \Delta_{\beta, \min }^{2}}{144^{2} m \log m}+\mathbb{E}\left[\bar{N}_{n}\left(\left(\frac{j_{\ell}}{m}, 1\right]\right)\right]$, it follows that

$$
\alpha_{n, \ell, 2}^{(1)} \leq 2 \exp \left(-\frac{n \xi^{2}}{2 \mathbb{E}\left[\bar{N}_{n}\left(\left(\frac{j_{\ell}}{m}, 1\right]\right)\right]+\frac{2}{3} \xi}\right) \rightarrow 0,
$$

as $n \rightarrow \infty$. Similarly, using (17) in Assumption 4, and (33) in Lemma 3 with $z=$ $\frac{\Delta_{j, \min } \Delta_{\beta, \min }}{72 \sqrt{m}}$, it implies that

$$
\alpha_{n, \ell, 2}^{(2)} \leq 2 \exp \left(-\frac{n z^{2}}{2 \mathbb{E}\left[\bar{N}_{n}\left(\left(\frac{j_{\ell}}{m}, \frac{\left\lceil\frac{j_{\ell}+j_{\ell+1}}{2}\right\rceil-1}{m}\right]\right)\right]+\frac{2}{3} z}\right) \rightarrow 0,
$$

as $n \rightarrow \infty$. Therefore, we conclude that $\mathbb{P}\left[A_{n, \ell, 2}\right] \rightarrow 0$, as $n \rightarrow \infty$. 
8.1.2 Step I.2. Prove: $\mathbb{P}\left[A_{n, \ell} \cap C_{n}^{\complement}\right] \rightarrow 0$, as $n \rightarrow \infty$.

Recall that $C_{n}^{\complement}=\left\{\max _{1 \leq k \leq L_{0}-1}\left|\hat{j}_{\ell}-j_{\ell}\right| \geq \frac{\Delta_{j, \min }}{2}\right\}$. We split $\mathbb{P}\left[A_{n, \ell} \cap C_{n}^{\complement}\right]$ in three terms as following

$$
\mathbb{P}\left[A_{n, \ell} \cap C_{n}^{\complement}\right]=\mathbb{P}\left[A_{n, \ell} \cap D_{n}^{(l)}\right]+\mathbb{P}\left[A_{n, \ell} \cap D_{n}^{(m)}\right]+\mathbb{P}\left[A_{n, \ell} \cap D_{n}^{(r)}\right]
$$

where

$$
\begin{aligned}
D_{n}^{(l)} & :=\left\{\text { there exists } \ell \in\left\{1, \ldots, L_{0}-1\right\}: \hat{j}_{\ell} \leq j_{\ell-1}\right\} \cap C_{n}^{\complement} \\
D_{n}^{(m)} & :=\left\{\text { for all } \ell \in\left\{1, \ldots, L_{0}-1\right\}: j_{\ell-1}<\hat{j}_{\ell}<j_{\ell+1}\right\} \cap C_{n}^{\complement}, \\
D_{n}^{(r)} & :=\left\{\text { there exists } \ell \in\left\{1, \ldots, L_{0}-1\right\}: \hat{j}_{\ell} \geq j_{\ell+1}\right\} \cap C_{n}^{\complement} .
\end{aligned}
$$

Let us first focus on $\mathbb{P}\left[A_{n, \ell} \cap D_{n}^{(m)}\right]$. Observe that

$$
\begin{aligned}
\mathbb{P}\left[A_{n, \ell} \cap D_{n}^{(m)}\right]=\mathbb{P}\left[A_{n, \ell}\right. & \left.\cap\left\{\hat{j}_{\ell+1}-j_{\ell} \geq \frac{\Delta_{j, \min }}{2}\right\} \cap D_{n}^{(m)}\right] \\
+ & \mathbb{P}\left[A_{n, \ell} \cap\left\{\hat{j}_{\ell+1}-j_{\ell}<\frac{\Delta_{j, \min }}{2}\right\} \cap D_{n}^{(m)}\right] .
\end{aligned}
$$

The fact that $0 \leq \hat{j}_{\ell+1}-j_{\ell}<\frac{\Delta_{j, \min }}{2}$ yields $j_{\ell+1}-\hat{j}_{\ell+1} \geq \frac{\Delta_{j, \min }}{2}$. Then, it is easy to see that $j_{\ell+1}-\hat{j}_{\ell+1}=\left(j_{\ell+1}-j_{\ell}\right)-\left(\hat{j}_{\ell+1}-j_{\ell}\right) \geq \Delta_{j, \min }-\frac{\Delta_{j, \min }}{2} \geq \frac{\Delta_{j, \min }}{2}$.

Hence

$$
\begin{aligned}
\mathbb{P}\left[A_{n, \ell} \cap D_{n}^{(m)}\right] \leq \mathbb{P}\left[A_{n, \ell} \cap\right. & \left.\left\{\hat{j}_{\ell+1}-j_{\ell} \geq \frac{\Delta_{j, \min }}{2}\right\} \cap D_{n}^{(m)}\right] \\
& +\mathbb{P}\left[A_{n, \ell} \cap\left\{j_{\ell+1}-\hat{j}_{\ell+1} \geq \frac{\Delta_{j, \min }}{2}\right\} \cap D_{n}^{(m)}\right] .
\end{aligned}
$$

Moreover, we note that

$$
\begin{aligned}
A_{n, \ell} \cap\left\{j_{\ell+1}-\right. & \left.\hat{j}_{\ell+1} \geq \frac{\Delta_{j, \min }}{2}\right\} \cap D_{n}^{(m)} \\
& \subset \bigcup_{r=\ell+1}^{L_{0}-2}\left\{j_{r}-\hat{j}_{r} \geq \frac{\Delta_{j, \min }}{2}\right\} \cap\left\{\hat{j}_{r+1}-j_{r} \geq \frac{\Delta_{j, \min }}{2}\right\} \cap D_{n}^{(m)} .
\end{aligned}
$$

Thus, we have

$$
\mathbb{P}\left[A_{n, \ell} \cap D_{n}^{(m)}\right] \leq \mathbb{P}\left[A_{n, \ell} \cap B_{\ell+1, \ell} \cap D_{n}^{(m)}\right]+\sum_{s=\ell+1}^{L_{0}-2} \mathbb{P}\left[C_{s, s} \cap B_{s+1, s} \cap D_{n}^{(m)}\right],
$$

where

$$
\left\{\begin{array}{l}
B_{p, q}=\left\{\left(\hat{j}_{p}-j_{q}\right) \geq \frac{\Delta_{j, \min }}{2}\right\}, \\
\text { with the convention } B_{L_{0}, L_{0}-1}=\left\{m-j_{L_{0}-1} \geq \frac{\Delta_{j, \min }}{2}\right\}, \\
C_{p, q}=\left\{\left(j_{p}-\hat{j}_{q}\right) \geq \frac{\Delta_{j, \min }}{2}\right\} .
\end{array}\right.
$$

Let us now prove that the first term in the right hand side of (36) goes to zero as $n$ tends to infinity, the arguments for the other terms being similar. Using (31) in Lemma 2 with $j=j_{\ell}$ and $j=\hat{j}_{\ell}+1$, on the one hand and (31) in Lemma 2 with $j=j_{\ell}+1$ and $j=\hat{j}_{\ell+1}$ on the other hand, we obtain, respectively

$$
\left|\hat{j}_{\ell}-j_{\ell}-2\right|\left|\hat{\beta}_{\hat{j}_{\ell+1}-1, m}-\beta_{0, j_{\ell}-1, m}\right| \leq \hat{w}_{\hat{j}_{\ell}+1, j_{\ell}}+\left|\sqrt{m} \bar{M}_{n}\left(\hat{j}_{\ell}+1 ; j_{\ell}-1\right)\right|,
$$


and

$$
\left|\hat{j}_{\ell+1}-j_{\ell}-2\right|\left|\hat{\beta}_{\hat{j}_{\ell+1}-1, m}-\beta_{0, j_{\ell+1}-1, m}\right| \leq \hat{w}_{j_{\ell}+1, \hat{j}_{\ell+1}}+\left|\sqrt{m} \bar{M}_{n}\left(j_{\ell}+1 ; \hat{j}_{\ell+1}-1\right)\right| .
$$

Besides, we have

$$
\begin{aligned}
& \left|\beta_{0, j_{\ell+1}-1, m}-\beta_{0, j_{\ell}-1, m}\right| \\
& =\left|\left(\hat{\beta}_{\hat{j}_{\ell+1}-1, m}-\beta_{0, j_{\ell}-1, m}\right)-\left(\hat{\beta}_{\hat{j}_{\ell+1}-1, m}-\beta_{0, j_{\ell+1}-1}\right)\right| \\
& \leq\left|\hat{\beta}_{\hat{j}_{\ell+1}-1, m}-\beta_{0, j_{\ell}-1, m}\right|+\left|\hat{\beta}_{\hat{j}_{\ell+1}-1, m}-\beta_{0, j_{\ell+1}-1, m}\right| \\
& \leq \frac{\hat{w}_{\hat{j}_{\ell}+1, j_{\ell}}}{\left|\hat{j}_{\ell}-j_{\ell}-2\right|}+\frac{\sqrt{m} \bar{M}_{n}\left(\hat{j}_{\ell}+1 ; j_{\ell}-1\right) \mid}{\left|\hat{j}_{\ell}-j_{\ell}-2\right|} \\
& \quad+\frac{\hat{w}_{j_{\ell}+1, \hat{j}_{\ell+1}}}{\left|\hat{j}_{\ell+1}-j_{\ell}-2\right|}+\frac{\left|\sqrt{m} \bar{M}_{n}\left(j_{\ell}+1 ; \hat{j}_{\ell+1}-1\right)\right|}{\left|\hat{j}_{\ell+1}-j_{\ell}-2\right|} \\
& \leq \frac{\hat{w}_{\hat{j}_{\ell}+1, j_{\ell}}}{\frac{m \varepsilon_{n}}{6}}+\frac{\sqrt{m} \bar{M}_{n}\left(\hat{j}_{\ell}+1 ; j_{\ell}-1\right) \mid}{\left|\hat{j}_{\ell}-j_{\ell}-2\right|} \\
& \quad+\frac{\hat{w}_{j_{\ell}+1, \hat{j}_{\ell+1}}}{\frac{\Delta_{j, \min }}{6}}+\frac{\left|\sqrt{m} \bar{M}_{n}\left(j_{\ell}+1 ; \hat{j}_{\ell+1}-1\right)\right|}{\left|\hat{j}_{\ell+1}-j_{\ell}-2\right|} .
\end{aligned}
$$

Define the event $E_{n, \ell}$ by

$$
\begin{aligned}
E_{n, \ell}=\left\{\left|\beta_{0, j_{\ell+1}-1, m}-\beta_{0, j_{\ell}-1, m}\right| \leq\right. & \frac{\hat{w}_{\hat{j}_{\ell}+1, j_{\ell}}}{\frac{m \varepsilon_{n}}{6}}+\frac{\hat{w}_{j_{\ell}+1, \hat{j}_{\ell+1}}}{\frac{\Delta_{j, \min }}{6}} \\
& +\left|\frac{\sqrt{m} \bar{M}_{n}\left(\hat{j}_{\ell}+1 ; j_{\ell}-1\right)}{\hat{j}_{\ell}-j_{\ell}-2}\right| \\
& \left.+\left|\frac{\sqrt{m} \bar{M}_{n}\left(j_{\ell}+1 ; \hat{j}_{\ell+1}-1\right)}{\hat{j}_{\ell+1}-j_{\ell}-2}\right|\right\} .
\end{aligned}
$$

We observe that $E_{n, \ell}$ occurs with probability one. Therefore, we obtain

$$
\begin{aligned}
& \mathbb{P}\left[A_{n, \ell} \cap B_{\ell+1, \ell} \cap D_{n}^{(m)}\right] \\
& \leq \mathbb{P}\left[E_{n, \ell} \cap\left\{\left(j_{\ell}-\hat{j}_{\ell}\right)>\frac{m \varepsilon_{n}}{2}\right\} \cap\left\{\left(\hat{j}_{\ell+1}-j_{\ell}\right) \geq \frac{\Delta_{j, \min }}{2}\right\}\right] \\
& \leq \mathbb{P}\left[\hat{w}_{\hat{j}_{\ell}+1, j_{\ell}} \geq \frac{m \varepsilon_{n}\left|\beta_{0, j_{\ell+1}-1, m}-\beta_{0, j_{\ell}-1, m}\right|}{24}\right] \\
&+\mathbb{P}\left[\hat{w}_{j_{\ell}+1, \hat{j}_{\ell+1}} \geq \frac{\Delta_{j, \min }\left|\beta_{0, j_{\ell+1}-1, m}-\beta_{0, j_{\ell}-1, m}\right|}{24}\right] \\
&+\mathbb{P}\left[\left\{\left|\frac{\sqrt{m} \bar{M}_{n}\left(\hat{j}_{\ell}+1 ; j_{\ell}-1\right)}{j_{\ell}-\hat{j}_{\ell}-2}\right|\right.\right. \\
&\left.\left.\geq \frac{\left|\beta_{0, j_{\ell+1}-1, m}-\beta_{0, j_{\ell}-1, m}\right|}{4}\right\} \bigcap\left\{j_{\ell}-\hat{j}_{\ell}-2 \geq \frac{m \varepsilon_{n}}{6}\right\}\right] \\
& \quad \mathbb{P}\left[\left\{\left|\frac{\sqrt{m} \bar{M}_{n}\left(j_{\ell}+1 ; \hat{j}_{\ell+1}-1\right)}{\hat{j}_{\ell+1}-j_{\ell}-2}\right|\right.\right. \\
&\left.\left.\geq \frac{\left|\beta_{0, j_{\ell+1}-1, m}-\beta_{0, j_{\ell}-1, m}\right|}{4}\right\} \bigcap\left\{\hat{j}_{\ell+1}-j_{\ell}-2 \geq \frac{\Delta_{j, \min }}{6}\right\}\right] . \\
&:= \theta_{n, \ell, 1}+\theta_{n, \ell, 2}+\theta_{n, \ell, 3}+\theta_{n, \ell, 4}
\end{aligned}
$$


We note

$$
\theta_{n, \ell, 1} \leq \mathbb{P}\left[\hat{w}_{\hat{j}_{\ell+1}} \geq \frac{m \varepsilon_{n} \Delta_{\beta, \min }}{48}\right] \leq \mathbb{P}\left[\hat{w}_{j_{\ell-1}+1}^{2} \geq \frac{m^{2} \varepsilon_{n}^{2} \Delta_{\beta, \min }^{2}}{48^{2}}\right]
$$

Using (16) in Assumption 4, and (34) in Lemma 3 with $\xi=\frac{n m \varepsilon_{n}^{2} \Delta_{\beta, \min }^{2}}{48^{2} \log m}+\mathbb{E}\left[\bar{N}_{n}\left(\left(\frac{j_{\ell-1}}{m}, 1\right]\right)\right]$, we get

$$
\theta_{n, \ell, 1} \leq 2 \exp \left(-\frac{n \xi^{2}}{2 \mathbb{E}\left[\bar{N}_{n}\left(\left(\frac{j \ell-1}{m}, 1\right]\right)\right]+\frac{2}{3} \xi}\right) \rightarrow 0,
$$

as $n \rightarrow \infty$. Analogously,

$$
\theta_{n, \ell, 2} \leq \mathbb{P}\left[\hat{w}_{\hat{j}_{\ell}+1} \geq \frac{\Delta_{j, \min } \Delta_{\beta, \min }}{48}\right] \leq \mathbb{P}\left[\hat{w}_{j_{\ell}+1}^{2} \geq \frac{\Delta_{j, \min }^{2} \Delta_{\beta, \min }^{2}}{48^{2}}\right]
$$

Using (17) in Assumption 4, and (34) in Lemma 3, with $\xi=\frac{n \Delta_{j, \min }^{2} \Delta_{\beta, \min }^{2}}{48^{2} m \log m}+\mathbb{E}\left[\bar{N}_{n}\left(\left(\frac{j_{\ell}}{m}, 1\right]\right)\right]$, we have

$$
\theta_{n, \ell, 2} \leq 2 \exp \left(-\frac{n \xi^{2}}{2 \mathbb{E}\left[\bar{N}_{n}\left(\left(\frac{j_{\ell}}{m}, 1\right]\right)\right]+\frac{2}{3} \xi}\right) \rightarrow 0
$$

as $n \rightarrow \infty$. Furthermore, using (33) in Lemma 3, we have

$$
\begin{aligned}
\theta_{n, \ell, 3} & \leq \mathbb{P}\left[\left|\bar{M}_{n}\left(\hat{j}_{\ell}+1 ; j_{\ell}-1\right)\right| \geq \frac{m \varepsilon_{n} \Delta_{\beta, \min }}{24 \sqrt{m}}\right] \\
& \leq 2 \exp \left(-\frac{n \psi_{n}^{2}}{2 \mathbb{E}\left[\bar{N}_{n}\left(\left(\frac{j_{\ell-1}+1}{m}, \frac{j_{\ell}-1}{m}\right]\right)\right]+\frac{2}{3} \psi_{n}}+\log m\right),
\end{aligned}
$$

where $\psi_{n}=\frac{\sqrt{m} \varepsilon_{n} \Delta_{\beta, \min }}{24}$. By (16) in Assumption 4, we get that $\theta_{n, \ell, 3} \rightarrow 0$, as $n \rightarrow \infty$. Similarly, using (33) in Lemma 3, we have

$$
\begin{aligned}
\theta_{n, \ell, 4} & \leq \mathbb{P}\left[\left|\bar{M}_{n}\left(j_{\ell}+1 ; \hat{j}_{\ell+1}-1\right)\right| \geq \frac{\Delta_{j, \min } \Delta_{\beta, \min }}{24 \sqrt{m}}\right] \\
& \leq 2 \exp \left(-\frac{n \delta_{n}^{2}}{2 \mathbb{E}\left[\bar{N}_{n}\left(\left(\frac{j_{\ell}}{m}, \frac{j_{\ell+2}-2}{m}\right]\right)\right]+\frac{2}{3} \delta_{n}}+\log m\right),
\end{aligned}
$$

where $\delta_{n}=\frac{\Delta_{j, \min } \Delta_{\beta, \min }}{24 \sqrt{m}}$. By (17) in Assumption 4, we get that $\theta_{n, \ell, 4} \rightarrow 0$, as $n \rightarrow \infty$. Consequently, we obtain $\mathbb{P}\left[A_{n, \ell} \cap B_{\ell+1, \ell} \cap D_{n}^{(m)}\right] \rightarrow 0$ as $n \rightarrow \infty$. Now, we have $\mathbb{P}\left[A_{n, \ell} \cap\right.$ $\left.D_{n}{ }^{(l)}\right] \leq \mathbb{P}\left[D_{n}{ }^{(l)}\right]$, and

$$
\begin{aligned}
\mathbb{P}\left[D_{n}{ }^{(l)}\right] & =\mathbb{P}\left[\left\{\exists \ell \in\left\{1, \ldots, L_{0}-1\right\}: \hat{j}_{\ell} \leq j_{\ell-1}\right\} \cap C_{n}^{\complement}\right] \\
& =\mathbb{P}\left[\left\{\bigcup_{\ell=1}^{L_{0}-1} \max \left\{1 \leq q \leq L_{0}-1: \hat{j}_{q} \leq j_{q-1}\right\}=\ell\right\} \cap C_{n}^{\complement}\right] \\
& =\sum_{\ell=1}^{L_{0}-1} \mathbb{P}\left[\left\{\max \left\{1 \leq q \leq L_{0}-1: \hat{j}_{q} \leq j_{q-1}\right\}=\ell\right\} \cap C_{n}^{\complement}\right] .
\end{aligned}
$$


We note that on the event $\left\{\max \left\{1 \leq q \leq L_{0}-1 ; \hat{j}_{q} \leq j_{q-1}\right\}=\ell\right\}$, it is clear to see that $\hat{j}_{\ell} \leq j_{\ell-1}$ and $\hat{j}_{q+1}>j_{q}$ for all $q=\ell, \ldots, L_{0}-1$. Then, it follows that

$$
\mathbb{P}\left[D_{n}{ }^{(l)}\right] \leq \sum_{\ell=1}^{L_{0}-1} 2^{\ell-1} \mathbb{P}\left[\bigcap_{q \geq \ell}^{L_{0}-1}\left\{\hat{j}_{\ell} \leq j_{\ell-1}\right\} \cap\left\{\hat{j}_{q+1}>j_{q}\right\}\right] .
$$

In addition, we note that

$$
\begin{aligned}
& \bigcap_{q \geq \ell}^{L_{0}-1}\left\{\hat{j}_{\ell} \leq j_{\ell-1}\right\} \cap\left\{\hat{j}_{q+1}>j_{q}\right\} \\
& \subset\left\{j_{\ell} \leq \hat{j}_{\ell}\right\} \cap\left(\left\{\hat{j}_{\ell+1}>\frac{j_{\ell}+j_{\ell+1}}{2}\right\} \cup\left\{\hat{j}_{\ell+1}<\frac{j_{\ell}+j_{\ell+1}}{2}\right\}\right) \\
& \quad \cap\left(\left\{\hat{j}_{\ell+2}>\frac{j_{\ell+1}+j_{\ell+2}}{2}\right\} \cup\left\{\hat{j}_{\ell+2}<\frac{j_{\ell+2}+j_{\ell+1}}{2}\right\}\right) \\
& \quad \cap \ldots \cap\left(\left\{\hat{j}_{L_{0}-1}>\frac{j_{L_{0}-2}+j_{L_{0}+1}}{2}\right\} \cup\left\{\hat{j}_{L_{0}-1}<\frac{j_{L_{0}-2}+j_{L_{0}+1}}{2}\right\}\right) \\
& \quad \cap\left(\left\{\hat{j}_{L_{0}}>\frac{\left.\left.j_{L_{0}-1}+j_{L_{0}}\right\} \cup\left\{\hat{j}_{L_{0}}<\frac{j_{L_{0}-1}+j_{L_{0}}}{2}\right\}\right)}{\subset\{}\left\{j_{\ell}-\hat{j}_{\ell}>\frac{\Delta_{j, \text { min }}}{2}\right\} \cap\left(\left\{\hat{j}_{\ell+1}-j_{\ell}>\frac{\Delta_{j, \text { min }}}{2}\right\} \cup\left\{j_{\ell+1}-\hat{j}_{\ell+1}>\frac{\Delta_{j, \text { min }}}{2}\right\}\right)\right.\right. \\
& \quad \cap\left(\left\{\hat{j}_{\ell+2}-j_{\ell+1}>\frac{\Delta_{j, \text { min }}}{2}\right\} \cup\left\{j_{\ell+2}-\hat{j}_{\ell+2}>\frac{\Delta_{j, \text { min }}}{2}\right\}\right) \\
& \quad \cap \ldots \cap\left(\left\{\hat{j}_{L_{0}-1}-j_{L_{0}-2}>\frac{\Delta_{j, \text { min }}}{2}\right\} \cup\left\{j_{L_{0}-2}-\hat{j}_{L_{0}-2}>\frac{\Delta_{j, \text { min }}}{2}\right\}\right) \\
& \quad \cap\left(\left\{\hat{j}_{L_{0}}-j_{L_{0}-1}>\frac{\Delta_{j, \text { min }}}{2}\right\} \cup\left\{j_{L_{0}-1}-\hat{j}_{L_{0}-1}>\frac{\Delta_{j, \text { min }}}{2}\right\}\right) \\
& \subset \bigcup_{q=\ell}^{L_{0}-2}\left(\left\{j_{q}-\hat{j}_{q}>\frac{\Delta_{j, \text { min }}}{2}\right\} \cap\left\{\hat{j}_{q+1}-j_{q}>\frac{\Delta_{j, \text { min }}}{2}\right\}\right) \cup\left\{j_{L_{0}-1}-\hat{j}_{L_{0}-1}>\frac{\Delta_{j, \text { min }}}{2}\right\} .
\end{aligned}
$$

Hence

$$
\begin{gathered}
\mathbb{P}\left[D_{n}{ }^{(l)}\right] \leq 2^{L_{0}-2} \sum_{\ell=1}^{L_{0}-2} \sum_{q=\ell}^{L_{0}-2} \mathbb{P}\left[\left\{\left(j_{q}-\hat{j}_{q}\right)>\frac{\Delta_{j, \min }}{2}\right\} \cap\left\{\hat{j}_{q+1}-j_{q}>\frac{\Delta_{j, \min }}{2}\right\}\right] \\
+2^{L_{0}-2} \mathbb{P}\left[\left\{j_{L_{0}-1}-\hat{j}_{L_{0}-1}>\frac{\Delta_{j, \min }}{2}\right\}\right] .
\end{gathered}
$$


Consider the first term of the sum in the right-hand side of (39). Using (37) and (38) with $\ell=q$, we obtain

$$
\begin{aligned}
& \mathbb{P}\left[\left\{j_{q}\right.\right.\left.\left.-\hat{j}_{q}>\frac{\Delta_{j, \min }}{2}\right\} \cap\left\{\hat{j}_{q+1}-j_{q}>\frac{\Delta_{j, \min }}{2}\right\}\right] \\
& \leq \mathbb{P}\left[\frac{\hat{w}_{\hat{j}_{q}+1, j_{q}}}{\left.\frac{\Delta_{j, \min }}{6} \geq \frac{\left|\beta_{0, j_{q+1}-1, m}-\beta_{0, j_{q}-1, m}\right|}{4}\right]}\right. \\
&+\mathbb{P}\left[\frac{\hat{w}_{j_{q}+1, \hat{j}_{q+1}}}{\frac{\Delta_{j, \min }}{6}} \geq \frac{\left|\beta_{0, j_{q+1}-1, m}-\beta_{0, j_{q}-1, m}\right|}{4}\right] \\
&+\mathbb{P}\left[\left\{\left|\frac{\sqrt{m} \bar{M}_{n}\left(\hat{j}_{q}+1 ; j_{q}-1\right)}{j_{q}-\hat{j}_{q}-2}\right|\right.\right. \\
&\left.\left.\geq \frac{\left|\beta_{0, j_{q+1}-1, m}-\beta_{0, j_{q}-1, m}\right|}{4}\right\} \bigcap\left\{j_{q}-\hat{j}_{q} \geq \frac{\Delta_{j, \min }}{2}\right\}\right] \\
&+\mathbb{P}\left[\left\{\left|\frac{\sqrt{m} \bar{M}_{n}\left(j_{q}+1 ; \hat{j}_{q+1}-1\right)}{\hat{j}_{q+1}-j_{q}-2}\right|\right.\right. \\
&\left.\left.\geq \frac{\left|\beta_{0, j_{q+1}-1, m}-\beta_{0, j_{q}-1, m}\right|}{4}\right\} \bigcap\left\{\hat{j}_{q+1}-j_{q} \geq \frac{\Delta_{j, \min }}{2}\right\}\right] .
\end{aligned}
$$

By (33)-(34) in Lemma 3, and (16)-(17) in Assumption 4, we show that for $s=1, \ldots, 4, \theta_{n, q, s} \rightarrow$ 0 , as $n$ tending to infinity. Then

$$
\mathbb{P}\left[\left\{j_{q}-\hat{j}_{q}>\frac{\Delta_{j, \min }}{2}\right\} \bigcap\left\{\hat{j}_{q+1}-j_{q}>\frac{\Delta_{j, \min }}{2}\right\}\right] \rightarrow 0 .
$$

Let us now consider the last term in the right hand of (39). Using the observations (37) and (38) with $\ell=L_{0}-1$ leads to

$$
\begin{aligned}
& \mathbb{P}\left[\left\{j_{L_{0}-1}-\hat{j}_{L_{0}-1}>\frac{\Delta_{j, \text { min }}}{2}\right\}\right] \\
& \leq \mathbb{P}\left[\frac{\hat{w}_{\hat{j}_{L_{0}-1}+1, j_{L_{0}-1}}}{\frac{m \varepsilon_{n}}{6}} \geq \frac{\left|\beta_{0, j_{L_{0}}-1, m}-\beta_{0, j_{L_{0}-1}-1, m}\right|}{4}\right] \\
&+\mathbb{P}\left[\frac{\hat{w}_{j_{L_{0}-1}+1, m}}{\left.\frac{\Delta_{j, \min }}{6} \geq \frac{\left|\beta_{0, j_{L_{0}}-1, m}-\beta_{0, j_{L_{0}-1}-1, m}\right|}{4}\right]}\right. \\
&+\mathbb{P}\left[\left\{\left|\frac{\sqrt{m} \bar{M}_{n}\left(\hat{j}_{L_{0}-1}+1 ; j_{L_{0}-1}-1\right)}{j_{L_{0}-1}-\hat{j}_{L_{0}-1}-2}\right|\right.\right. \\
&\left.\left.\geq \frac{\left|\beta_{0, j_{L_{0}}-1, m}-\beta_{0, j_{L_{0}-1}-1, m}\right|}{4}\right\} \bigcap\left\{j_{L_{0}-1}-\hat{j}_{L_{0}-1} \geq \frac{\Delta_{j, \min }}{2}\right\}\right] \\
&+\mathbb{P}\left[\left\{\left|\frac{\sqrt{m} \bar{M}_{n}\left(j_{L_{0}-1}+1 ; m-1\right)}{m-j_{L_{0}-1}-2}\right| \geq \frac{\left|\beta_{0, j_{L_{0}}-1, m}-\beta_{0, j_{L_{0}-1}-1, m}\right|}{4}\right\}\right]
\end{aligned}
$$

By (33)-(34) in Lemma 3, and (16)-(17) in Assumption 4, we show that for $s=1, \ldots, 4$, we obtain $\theta_{n, L_{0}-1, s} \rightarrow 0$, as $n \rightarrow \infty$. Then

$$
\mathbb{P}\left[\left\{j_{L_{0}-1}-\hat{j}_{L_{0}-1}>\frac{\Delta_{j, \min }}{2}\right\} \bigcap\left\{m-j_{L_{0}-1}>\frac{\Delta_{j, \min }}{2}\right\}\right] \rightarrow 0 .
$$


This implies that $\mathbb{P}\left[D_{n}{ }^{(l)}\right] \rightarrow 0$, as $n \rightarrow \infty$. Similarly, we prove that $\mathbb{P}\left[D_{n}{ }^{(r)}\right] \rightarrow 0$, as $n \rightarrow \infty$ which yields that $\mathbb{P}\left[A_{n, \ell} \cap C_{n}^{\complement}\right] \rightarrow 0$, as $n \rightarrow \infty$. This concludes the proof of Theorem 3, up to the case $\left\{\hat{j}_{\ell}>j_{\ell}\right\}$ for a fixed $\ell \in\left\{1, \ldots, L_{0}-1\right\}$ which is given in Appendix B.

\section{Proof of Theorem 4}

This proof is based on the same arguments in the proof of Theorem 3. Let $\mathcal{T}_{0}^{\text {approx }}=$ $\left\{\frac{j_{1}}{m}, \ldots, \frac{j_{L_{0}-1}}{m}\right\}$ be the set of the true approximate change-points. First, we note that

$$
\left.\mathbb{P}\left[\mathcal{E}\left(\hat{\mathcal{T}} \| \mathcal{T}_{0}\right)>\varepsilon_{n}\right] \leq \mathbb{P}\left[\mathcal{E}\left(\hat{\mathcal{T}} \| \mathcal{T}_{0}^{\text {approx }}\right)>\varepsilon_{n}\right]+\mathbb{P}\left[\mathcal{E}\left(\mathcal{T}_{0}^{\text {approx }}\right) \| \mathcal{T}_{0}\right)>\varepsilon_{n}\right]
$$

Obviously, since $m \varepsilon_{n} \geq 6$, we have $\left.\mathbb{P}\left[\mathcal{E}\left(\mathcal{T}_{0}^{\text {approx }}\right) \| \mathcal{T}_{0}\right)>\varepsilon_{n}\right]=0$. It is clear to remark that the inequality $\hat{L} \leq m$ holds true. In order to prove that

$$
\mathbb{P}\left[\left\{\mathcal{E}\left(\hat{\mathcal{T}} \| \mathcal{T}_{0}^{\text {approx }}\right)>\varepsilon_{n}\right\} \bigcap\left\{\hat{L} \geq L_{0}-1\right\}\right] \rightarrow 0,
$$

as $n \rightarrow \infty$, it is enough to prove that

$$
\mathbb{P}\left[\left\{\mathcal{E}\left(\hat{\mathcal{T}} \| \mathcal{T}_{0}^{\text {approx }}\right)>\varepsilon_{n}\right\} \bigcap\left\{L_{0}-1 \leq \hat{L} \leq m\right\}\right] \rightarrow 0
$$

as $n \rightarrow \infty$. We have that

$$
\begin{aligned}
& \mathbb{P}\left[\left\{\mathcal{E}\left(\hat{\mathcal{T}} \| \mathcal{T}_{0}^{\text {approx }}\right)>\varepsilon_{n}\right\} \bigcap\left\{L_{0}-1 \leq \hat{L} \leq m\right\}\right] \\
& \leq \mathbb{P}\left[\left\{\mathcal{E}\left(\hat{\mathcal{T}} \| \mathcal{T}_{0}^{\text {approx }}\right)>\varepsilon_{n}\right\} \bigcap\left\{\mathbb{1}_{\hat{L}=L_{0}-1}\right\}\right]+\mathbb{P}\left[\left\{\mathcal{E}\left(\hat{\mathcal{T}} \| \mathcal{T}_{0}^{\text {approx }}\right)>\varepsilon_{n}\right\} \bigcap\left\{\mathbb{1}_{\hat{L}>L_{0}-1}\right\}\right] \\
& \leq \mathbb{P}\left[\left\{\mathcal{E}\left(\hat{\mathcal{T}} \| \mathcal{T}_{0}^{\text {approx }}\right)>\varepsilon_{n}\right\} \bigcap\left\{\mathbb{1}_{\hat{L}=L_{0}-1}\right\}\right]+\sum_{L=L_{0}}^{m} \mathbb{P}\left[\mathcal{E}\left(\hat{\mathcal{T}} \| \mathcal{T}_{0}^{\text {approx }}\right)>\varepsilon_{n}\right] \\
& \leq \mathbb{P}\left[\left\{\mathcal{E}\left(\hat{\mathcal{T}} \| \mathcal{T}_{0}^{\text {approx }}\right)>\varepsilon_{n}\right\} \bigcap\left\{\mathbb{1}_{\hat{L}=L_{0}-1}\right\}\right] \\
& \quad+\sum_{L=L_{0}}^{m} \sum_{\ell=1}^{L_{0}-1} \mathbb{P}\left[\forall q \in\{1, \ldots, L\},\left|\frac{\hat{j}_{q}}{m}-\frac{j_{\ell}}{m}\right|>\varepsilon_{n}\right]
\end{aligned}
$$

The first term of the right-hand side of (40) tends to zero as $n$ tends to infinity since it is upper bounded by $\mathbb{P}\left[\max _{1 \leq \ell \leq L_{0}-1}\left|\hat{j}_{\ell}-j_{\ell}\right|>m \varepsilon_{n}\right]$ which tends to zero by the proof of Theorem 3. Let us now focus on the second term on the right-hand side of (40). Note that

$$
\sum_{L=L_{0}}^{m} \sum_{\ell=1}^{L_{0}-1} \mathbb{P}\left[\forall 1 \leq q \leq L,\left|\hat{j}_{q}-j_{\ell}\right|>m \varepsilon_{n}\right]:=\sum_{L=L_{0}}^{m} \sum_{\ell=1}^{L_{0}-1} \mathbb{P}\left[R_{n, \ell, 1}\right]+\mathbb{P}\left[R_{n, \ell, 2}\right]+\mathbb{P}\left[R_{n, \ell, 3}\right],
$$

where

$$
\begin{aligned}
& R_{n, \ell, 1}:=\left\{\forall 1 \leq q \leq L:\left|\hat{j}_{q}-j_{\ell}\right|>m \varepsilon_{n} \text { and } \hat{j}_{q}<j_{\ell}\right\} \\
& R_{n, \ell, 2}:=\left\{\forall 1 \leq q \leq L:\left|\hat{j}_{q}-j_{\ell}\right|>m \varepsilon_{n} \text { and } \hat{j}_{q}>j_{\ell}\right\} \\
& R_{n, \ell, 3}:=\left\{\exists 1 \leq q \leq L-1:\left\{\left|\hat{j}_{q}-j_{\ell}\right|>m \varepsilon_{n}\right\},\left\{\left|\hat{j}_{q+1}-j_{\ell}\right|>m \varepsilon_{n}\right\}, \text { and }\left\{\hat{j}_{q}<j_{\ell}<\hat{j}_{q+1}\right\}\right\} .
\end{aligned}
$$


Note that

$$
\mathbb{P}\left[R_{n, \ell, 1}\right]=\mathbb{P}\left[R_{n, \ell, 1} \cap\left\{\hat{j}_{L}>j_{\ell-1}\right\}\right]+\mathbb{P}\left[R_{n, \ell, 1} \cap\left\{\hat{j}_{L} \leq j_{\ell-1}\right\}\right] .
$$

By applying (31) in Lemma 2 with $j=j_{\ell}$ and with $j=\hat{j}_{L}+1$ in the case where $\hat{j}_{L}>j_{\ell-1}$, it follows that, with probability one,

$$
\begin{aligned}
& \mid\left(j_{\ell}-\hat{j}_{L}-2\right)\left(\left(\beta_{0, j_{\ell}-1, m}-\beta_{0, j_{\ell+1}-1, m}\right)\right. \\
& \left.+\left(\beta_{0, j_{\ell+1}-1, m}-\hat{\beta}_{\hat{j}_{L+1}-1, m}\right)\right) \\
& \quad+\sqrt{m} \bar{M}_{n}\left(\hat{j}_{L}+1 ; j_{\ell}-1\right) \mid \leq \hat{w}_{\hat{j}_{L}+1, j_{\ell}} .
\end{aligned}
$$

Thus

$$
\begin{aligned}
\mathbb{P} & {\left[R_{n, \ell, 1} \cap\left\{\hat{j}_{L}>j_{\ell-1}\right\}\right] } \\
\leq & \mathbb{P}\left[\left\{\frac{\left.\left.\hat{w}_{\hat{j}_{L}+1, j_{\ell}} \geq \frac{\left|\beta_{0, j_{\ell+1}-1, m}-\beta_{0, j_{\ell}-1, m}\right|}{m \varepsilon_{n}-2}\right\} \cap\left\{\hat{j}_{L}>j_{\ell-1}\right\}\right]}{3} \quad\right.\right. \\
& +\mathbb{P}\left[\left\{\left|\hat{\beta}_{\hat{j}_{L+1}-1, m}-\beta_{0, j_{\ell+1}-1, m}\right| \geq \frac{\left|\beta_{0, j_{\ell+1}-1, m}-\beta_{0, j_{\ell}-1, m}\right|}{3}\right\}\right] \\
& +\mathbb{P}\left[\left\{\left|\frac{\bar{M}_{n}\left(\hat{j}_{L}+1 ; j_{\ell}-1\right)}{j_{\ell}-\hat{j}_{L}-2}\right| \geq \frac{\left|\beta_{0, j_{\ell+1}-1, m}-\beta_{0, j_{\ell}-1, m}\right|}{3 \sqrt{m}}\right\} \cap\left\{\left|j_{\ell}-\hat{j}_{L}\right| \geq m \varepsilon_{n}\right\}\right] \\
:= & \mathbb{P}\left[R_{n, \ell, 1}^{(1)}\right]+\mathbb{P}\left[R_{n, \ell, 1}^{(2)}\right]+\mathbb{P}\left[R_{n, \ell, 1}^{(3)}\right] .
\end{aligned}
$$

Using (16) in Assumption 4, and (33)-(34) in Lemma 3 with $\xi=\frac{n m \varepsilon_{n}^{2} \Delta_{\beta, \min }^{2}}{36^{2} \log m}+\mathbb{E}\left[\bar{N}_{n}\left(\left(\frac{j_{\ell-1}}{m}, 1\right]\right)\right]$, we prove that $\sum_{L=L_{0}}^{m} \sum_{\ell=1}^{L_{0}-1} \mathbb{P}\left[R_{n, \ell, 1}^{(3)}\right] \rightarrow 0$, as $n \rightarrow \infty$. Let us now consider to $\mathbb{P}\left[R_{n, \ell, 2}^{(2)}\right]$. Using (31) in Lemma 2 with $j=j_{\ell}+1$ and with $j=j_{\ell+1}$, we get

$$
\left(j_{\ell+1}-j_{\ell}-2\right)\left|\hat{\beta}_{\hat{j}_{L+1}-1, m}-\beta_{0, j_{\ell+1}-1, m}\right| \leq \hat{w}_{j_{\ell}+1, j_{\ell+1}}+\left|\sqrt{m} \bar{M}_{n}\left(j_{\ell}+1 ; j_{\ell+1}-1\right)\right| .
$$

Therefore, we may upper bound $\mathbb{P}\left[R_{n, \ell, 2}^{(2)}\right]$ as follows:

$$
\begin{aligned}
& \mathbb{P}\left[\left|\hat{\beta}_{\hat{j}_{L+1}-1, m}-\beta_{0, j_{\ell+1}-1, m}\right| \geq \frac{\left|\beta_{0, j_{\ell+1}-1, m}-\beta_{0, j_{\ell}-1, m}\right|}{3}\right] \\
& \leq \mathbb{P}\left[\hat{w}_{j_{\ell}+1, j_{\ell+1}} \geq\left(j_{\ell+1}-j_{\ell}-2\right) \frac{\left|\beta_{0, j_{\ell+1}-1, m}-\beta_{0, j_{\ell}-1, m}\right|}{6}\right] \\
&+\mathbb{P}\left[\left|\frac{\bar{M}_{n}\left(j_{\ell}+1 ; j_{\ell+1}-1\right)}{j_{\ell+1}-j_{\ell}-2}\right| \geq \frac{\left|\beta_{0, j_{\ell+1}-1, m}-\beta_{0, j_{\ell}-1, m}\right|}{6 \sqrt{m}}\right] .
\end{aligned}
$$

By using Lemma 2, and (16)-(17) in Assumption 4, we conclude that $\sum_{L=L_{0}}^{m} \sum_{\ell=1}^{L_{0}-1} \mathbb{P}\left(R_{n, \ell, 1}^{(2)}\right) \rightarrow$ 0 , as $n \rightarrow \infty$. Analogously, it can be shown that $\sum_{L=L_{0}}^{m} \sum_{\ell=1}^{L_{0}-1} \mathbb{P}\left[R_{n, \ell, 1} \cap\left\{\hat{j}_{L} \leq j_{\ell-1}\right\}\right] \rightarrow$ 0 , as $n \rightarrow \infty$. Moreover, we prove, similarly, that $\sum_{L=L_{0}}^{m} \sum_{\ell=1}^{L_{0}-1} \mathbb{P}\left[R_{n, \ell, 2}\right] \rightarrow 0$, as $n \rightarrow \infty$. Let us now focus on $\sum_{L=L_{0}}^{m} \sum_{\ell=1}^{L_{0}-1} \mathbb{P}\left[R_{n, \ell, 3}\right]$. Note that $\mathbb{P}\left[R_{n, \ell, 3}\right]$ can be split in four terms as follows:

$$
\mathbb{P}\left[R_{n, \ell, 3}\right]=\mathbb{P}\left[R_{n, \ell, 3}^{(1)}\right]+\mathbb{P}\left[R_{n, \ell, 3}^{(2)}\right]+\mathbb{P}\left[R_{n, \ell, 3}^{(3)}\right]+\mathbb{P}\left[R_{n, \ell, 3}^{(4)}\right],
$$


where

$$
\begin{aligned}
& R_{n, \ell, 3}^{(1)}:=R_{n, \ell, 3} \cap\left\{j_{\ell-1}<\hat{j}_{q}<\hat{j}_{q+1}<j_{\ell+1}\right\} \\
& R_{n, \ell, 3}^{(2)}:=R_{n, \ell, 3} \cap\left\{j_{\ell-1}<\hat{j}_{q}<j_{\ell+1}, \hat{j}_{q+1} \geq j_{\ell+1}\right\} \\
& R_{n, \ell, 3}^{(3)}:=R_{n, \ell, 3} \cap\left\{\hat{j}_{q} \leq j_{\ell-1}, j_{\ell-1}<\hat{j}_{q+1}<j_{\ell+1}\right\} \\
& R_{n, \ell, 3}^{(4)}:=R_{n, \ell, 3} \cap\left\{\hat{j}_{q} \leq j_{\ell-1}, j_{\ell+1} \leq \hat{j}_{q+1}\right\} .
\end{aligned}
$$

We have to use Lemma 2 twice. For $\mathbb{P}\left[R_{n, \ell, 3}^{(1)}\right]$, we first use (31) in Lemma 2 with $j=j_{\ell}$ and $j=\hat{j}_{q}+1$, respectively, which gives with probability one

$$
\left|\left(j_{\ell}-\hat{j}_{q}-2\right)\left(\beta_{0, j_{\ell}-1, m}-\hat{\beta}_{\hat{j}_{q+1}-1, m}\right)+\sqrt{m} \bar{M}_{n}\left(\hat{j}_{q}+1 ; j_{\ell}-1\right)\right| \leq \hat{w}_{\hat{j}_{q}+1, j_{\ell}} .
$$

Thus,

$$
\left|\beta_{0, j_{\ell}-1, m}-\hat{\beta}_{\hat{j}_{q+1}-1, m}\right| \leq \frac{\hat{w}_{\hat{j}_{q}+1, j_{\ell}}}{j_{\ell}-\hat{j}_{q}-2}+\left|\frac{\sqrt{m} \bar{M}_{n}\left(\hat{j}_{q}+1 ; j_{\ell}-1\right)}{j_{\ell}-\hat{j}_{q}-2}\right| .
$$

Second, we use (31) in Lemma 2 with $j=j_{\ell}+1$ and $j=\hat{j}_{q+1}$, respectively, to get with probability one

$$
\left|\left(\hat{j}_{q+1}-j_{\ell}-2\right)\left\{\left(\beta_{0, j_{\ell+1}-1, m}-\hat{\beta}_{\hat{j}_{q+1}-1, m}\right)\right\}+\sqrt{m} \bar{M}_{n}\left(j_{\ell}+1 ; \hat{j}_{q+1}-1\right)\right| \leq \hat{w}_{j_{\ell}+1, \hat{j}_{q+1}} .
$$

Hence

$$
\left|\beta_{0, j_{\ell+1}-1, m}-\hat{\beta}_{\hat{j}_{q+1}-1, m}\right| \leq \frac{\hat{w}_{j_{\ell}+1, \hat{j}_{q+1}}}{\hat{j}_{q+1}-j_{\ell}-2}+\left|\frac{\sqrt{m} \bar{M}_{n}\left(j_{\ell}+1 ; \hat{j}_{q+1}-1\right)}{\hat{j}_{q+1}-j_{\ell}-2}\right| .
$$

Define the event

$$
\begin{gathered}
Q_{n, \ell, 3}^{(1)}=\left\{\left|\beta_{0, j_{\ell+1}-1, m}-\beta_{0, j_{\ell}-1, m}\right| \leq \frac{\hat{w}_{\hat{j}_{q}+1, j_{\ell}}}{\left|j_{\ell}-\hat{j}_{q}-2\right|}+\left|\frac{\sqrt{m} \bar{M}_{n}\left(\hat{j}_{q}+1 ; j_{\ell}-1\right)}{j_{\ell}-\hat{j}_{q}}\right|\right. \\
\left.+\frac{\hat{w}_{j_{\ell}+1, \hat{j}_{q+1}}}{\left|\hat{j}_{q+1}-j_{\ell}-2\right|}+\left|\frac{\sqrt{m} \bar{M}_{n}\left(j_{\ell}+1 ; \hat{j}_{q+1}-1\right)}{\hat{j}_{q+1}-j_{\ell}-2}\right|\right\} \\
\subset\left\{\left|\beta_{0, j_{\ell+1}-1, m}-\beta_{0, j_{k}-1, m}\right| \leq \frac{\hat{w}_{\hat{j}_{q}+1, j_{\ell}}}{m \varepsilon_{n}-2}+\left|\frac{\sqrt{m} \bar{M}_{n}\left(\hat{j}_{q}+1 ; j_{\ell}-1\right)}{m \varepsilon_{n}-2}\right|\right. \\
+\frac{\left.\hat{w}_{j_{\ell}+1, \hat{j}_{q+1}}+\left|\frac{\sqrt{m} \bar{M}_{n}\left(j, 1 ; \hat{j}_{q+1}-1\right)}{m \varepsilon_{n}-2}\right|\right\} .}{m \varepsilon_{n}-2}
\end{gathered}
$$

We observe that the event $Q_{n, \ell, 3}^{(1)}$ occurs with probability one, so

$$
\begin{aligned}
\mathbb{P}\left[R_{n, \ell, 3}^{(1)}\right]= & \mathbb{P}\left[R_{n, \ell, 3}^{(1)} \cap Q_{n, \ell, 3}^{(1)}\right] \\
\leq \quad & \mathbb{P}\left[\frac{\hat{w}_{\hat{j}_{q}+1, j_{\ell}}}{m \varepsilon_{n}-2} \geq \frac{\left|\beta_{0, j_{\ell+1}-1, m}-\beta_{0, j_{\ell}-1, m}\right|}{4}\right] \\
& +\mathbb{P}\left[\frac{\hat{w}_{j_{\ell}+1, \hat{j}_{q+1}}}{m \varepsilon_{n}-2} \geq \frac{\left|\beta_{0, j_{\ell+1}-1, m}-\beta_{0, j_{\ell}-1, m}\right|}{4}\right] \\
& +\mathbb{P}\left[\left|\frac{\sqrt{m} \bar{M}_{n}\left(\hat{j}_{q}+1 ; j_{\ell}-1\right)}{m \varepsilon_{n}-2}\right| \geq \frac{\left|\beta_{0, j_{\ell+1}-1, m}-\beta_{0, j_{\ell}-1, m}\right|}{4}\right] \\
& +\mathbb{P}\left[\left|\frac{\sqrt{m} \bar{M}_{n}\left(j_{\ell}+1 ; \hat{j}_{q+1}-1\right)}{m \varepsilon_{n}-2}\right| \geq \frac{\left|\beta_{0, j_{\ell+1}-1, m}-\beta_{0, j_{\ell}-1, m}\right|}{4}\right] .
\end{aligned}
$$


Using Lemmas 2 and 3, and (16)-(17) from Assumption 4, each term of the last inequality goes to zero, as $n \rightarrow \infty$. For $\mathbb{P}\left[R_{n, \ell, 3}^{(2)}\right]$, we apply Lemma 2 with $j=j_{\ell}$ and $j=\hat{j}_{q}+1$ to obtain (41) and then with $j=j_{\ell}+1$ and $j=j_{\ell+1}$ to get

$$
\left|\left(j_{\ell+1}-j_{\ell}-2\right)\left\{\left(\beta_{0, j_{\ell+1}-1, m}-\hat{\beta}_{\hat{j}_{q+1}-1, m}\right)\right\}+\sqrt{m} \bar{M}_{n}\left(j_{\ell}+1 ; j_{\ell+1}-1\right)\right| \leq \hat{w}_{j_{\ell+1, j_{\ell+1}}} .
$$

It follows that event $Q_{n, \ell, 3}^{(2)}$ occurs with probability one, where

$$
\begin{gathered}
Q_{n, \ell, 3}^{(2)}=\left\{\left|\beta_{0, j_{\ell+1}-1, m}-\beta_{0, j_{\ell}-1, m}\right| \leq \frac{\hat{w}_{\hat{j}_{q}+1, j_{\ell}}}{\left|j_{\ell}-\hat{j}_{q}-2\right|}+\left|\frac{\sqrt{m} \bar{M}_{n}\left(\hat{j}_{q}+1 ; j_{\ell}-1\right)}{j_{\ell}-\hat{j}_{q}}\right|\right. \\
\left.+\frac{\hat{w}_{j_{\ell}+1, j_{\ell+1}}}{\left|j_{\ell+1}-j_{\ell}-2\right|}+\left|\frac{\sqrt{m} \bar{M}_{n}\left(j_{\ell}+1 ; j_{\ell+1}-1\right)}{j_{\ell+1}-j_{\ell}-2}\right|\right\} \\
\subset\left\{\left|\beta_{0, j_{\ell+1}-1, m}-\beta_{0, j_{k}-1, m}\right| \leq \frac{\hat{w}_{\hat{j}_{q}+1, j_{\ell}}}{m \varepsilon_{n}-2}+\left|\frac{\sqrt{m} \bar{M}_{n}\left(\hat{j}_{q}+1 ; j_{\ell}-1\right)}{m \varepsilon_{n}-2}\right|\right. \\
+\frac{\left.\hat{w}_{j_{\ell}+1, j_{\ell+1}}+\left|\frac{\sqrt{m} \bar{M}_{n}\left(j_{\ell}+1 ; \hat{j}_{q+1}-1\right)}{\Delta_{j, \min }-2}\right|\right\} .}{\Delta_{j, \min }-2},
\end{gathered}
$$

Then

$$
\begin{aligned}
\mathbb{P}\left[R_{n, \ell, 3}^{(2)}\right]= & \mathbb{P}\left[R_{n, \ell, 3}^{(2)} \cap Q_{n, \ell, 3}^{(2)}\right] \\
\leq \quad & \mathbb{P}\left[\frac{\hat{w}_{\hat{j}_{q}+1, j_{\ell}}}{m \varepsilon_{n}-2} \geq \frac{\left|\beta_{0, j_{\ell+1}-1, m}-\beta_{0, j_{\ell}-1, m}\right|}{4}\right] \\
& +\mathbb{P}\left[\frac{\hat{w}_{j_{\ell}+1, j_{\ell+1}}}{\Delta_{j, \min }-2} \geq \frac{\left|\beta_{0, j_{\ell+1}-1, m}-\beta_{0, j_{\ell}-1, m}\right|}{4}\right] \\
& +\mathbb{P}\left[\left|\frac{\sqrt{m} \bar{M}_{n}\left(\hat{j}_{q}+1 ; j_{\ell}-1\right)}{m \varepsilon_{n}-2}\right| \geq \frac{\left|\beta_{0, j_{\ell+1}-1, m}-\beta_{0, j_{\ell}-1, m}\right|}{4}\right] \\
& +\mathbb{P}\left[\left|\frac{\sqrt{m} \bar{M}_{n}\left(j_{\ell}+1 ; j_{\ell+1}-1\right)}{\Delta_{j, \min }-2}\right| \geq \frac{\left|\beta_{0, j_{\ell+1}-1, m}-\beta_{0, j_{\ell}-1, m}\right|}{4}\right] .
\end{aligned}
$$

Using Lemmas 2 and 3, (16)-(17) in Assumption 4, each term of the last inequality tends to zero as $n \rightarrow+\infty$. For $\mathbb{P}\left[R_{n, \ell, 3}^{(3)}\right]$, we first use Lemma 2 with $j=j_{\ell-1}+1$ and $j=j_{\ell}$ to get

$$
\left|\left(j_{\ell}-j_{\ell-1}-2\right)\left(\beta_{0, j_{\ell}-1, m}-\hat{\beta}_{\hat{j}_{q+1}-1, m}\right)+\sqrt{m} \bar{M}_{n}\left(j_{\ell-1}+1 ; j_{\ell}-1\right)\right| \leq \hat{w}_{j_{\ell-1}+1, j_{\ell}} .
$$

And then with $j=j_{\ell}+1$ and $j=\hat{j}_{q+1}$, to obtain

$$
\left|\left(\hat{j}_{q+1}-j_{\ell}-2\right)\left(\beta_{0, j_{\ell+1}-1, m}-\hat{\beta}_{\hat{j}_{q+1}-1, m}\right)+\sqrt{m} \bar{M}_{n}\left(j_{\ell}+1 ; \hat{j}_{q+1}-1\right)\right| \leq \hat{w}_{j_{\ell}+1, \hat{j}_{q+1}} .
$$


Hence the event $Q_{n, \ell, 3}^{(3)}$ occurs with probability one, where

$$
\begin{gathered}
Q_{n, \ell, 3}^{(3)}=\left\{\left|\beta_{0, j_{\ell+1}-1, m}-\beta_{0, j_{\ell}-1, m}\right| \leq \frac{\hat{w}_{j_{\ell-1}+1, j_{\ell}}}{\left|j_{\ell}-j_{\ell-1}-2\right|}+\left|\frac{\sqrt{m} \bar{M}_{n}\left(j_{\ell-1}+1 ; j_{\ell}-1\right)}{j_{\ell}-j_{\ell-1}-2}\right|\right. \\
\left.+\frac{\hat{w}_{j_{\ell}+1, \hat{j}_{q+1}}}{\left|\hat{j}_{q+1}-j_{\ell}-2\right|}+\left|\frac{\sqrt{m} \bar{M}_{n}\left(j_{\ell}+1 ; \hat{j}_{q+1}-1\right)}{\hat{j}_{q+1}-j_{\ell}-2}\right|\right\} \\
\subset\left\{\left|\beta_{0, j_{\ell+1}-1, m}-\beta_{0, j_{k}-1, m}\right| \leq \frac{\hat{w}_{j_{\ell-1}+1, j_{\ell}}}{\Delta_{j, \min }-2}+\left|\frac{\sqrt{m} \bar{M}_{n}\left(j_{\ell-1}+1 ; j_{\ell}-1\right)}{\Delta_{j, \min }-2}\right|\right. \\
+\frac{\left.\hat{w}_{j_{\ell}+1, \hat{j}_{q+1}}+\left|\frac{\sqrt{m} \bar{M}_{n}\left(j_{\ell}+1 ; \hat{j}_{q+1}-1\right)}{m \varepsilon_{n}-2}\right|\right\} .}{m}-2
\end{gathered}
$$

Then

$$
\begin{aligned}
\mathbb{P}\left[R_{n, \ell, 3}^{(3)}\right]= & \mathbb{P}\left[R_{n, \ell, 3}^{(3)} \cap Q_{n, \ell, 3}^{(3)}\right] \\
\leq \quad & \mathbb{P}\left[\frac{\hat{w}_{j_{\ell-1}+1, j_{\ell}}}{\Delta_{j, \min }-2} \geq \frac{\left|\beta_{0, j_{\ell+1}-1, m}-\beta_{0, j_{\ell}-1, m}\right|}{4}\right] \\
& +\mathbb{P}\left[\frac{\hat{w}_{j_{\ell}+1, \hat{j}_{q+1}}}{m \varepsilon_{n}-2} \geq \frac{\left|\beta_{0, j_{\ell+1}-1, m}-\beta_{0, j_{\ell}-1, m}\right|}{4}\right] \\
& +\mathbb{P}\left[\left|\frac{\sqrt{m} \bar{M}_{n}\left(j_{\ell-1}+1 ; j_{\ell}-1\right)}{\Delta_{j, \min }-2}\right| \geq \frac{\left|\beta_{0, j_{\ell+1}-1, m}-\beta_{0, j_{\ell}-1, m}\right|}{4}\right] \\
& +\mathbb{P}\left[\left|\frac{\sqrt{m} \bar{M}_{n}\left(j \ell+1 ; \hat{j}_{q+1}-1\right)}{m \varepsilon_{n}-2}\right| \geq \frac{\left|\beta_{0, j_{\ell+1}-1, m}-\beta_{0, j_{\ell}-1, m}\right|}{4}\right] .
\end{aligned}
$$

By Lemmas 2 and 3, and (16)-(17) in Assumption 4, it implies that each term of the last inequality tends to zero as $n \rightarrow+\infty$. Finally, for $\mathbb{P}\left[R_{n, \ell, 3}^{(4)}\right]$, we first use Lemma 2 with $j=j_{\ell-1}+1$ and $j=j_{\ell}$ to obtain

$$
\left|\left(j_{\ell}-j_{\ell-1}-2\right)\left\{\left(\beta_{0, j_{\ell}-1, m}-\hat{\beta}_{\hat{j}_{q+1}-1, m}\right)\right\}+\sqrt{m} \bar{M}_{n}\left(j_{\ell-1}+1 ; j_{\ell}-1\right)\right| \leq \hat{w}_{j_{\ell-1}+1, j_{\ell}} .
$$

Second, we use Lemma 2 with $j=j_{\ell}+1$ and $j=j_{\ell+1}$ to obtain

$$
\left|\left(j_{\ell+1}-j_{\ell}-2\right)\left(\beta_{0, j_{\ell+1}-1, m}-\hat{\beta}_{\hat{j}_{q+1}-1, m}\right)+\sqrt{m} \bar{M}_{n}\left(j_{\ell}+1 ; j_{\ell+1}-1\right)\right| \leq \hat{w}_{j_{\ell}+1, j_{\ell+1}} .
$$

It follows that the event $Q_{n, \ell, 3}^{(4)}$ occurs with probability one, where

$$
\begin{aligned}
& Q_{n, \ell, 3}^{(4)}=\left\{\left|\beta_{0, j_{\ell+1}-1, m}-\beta_{0, j_{\ell}-1, m}\right| \leq \frac{\hat{w}_{j_{\ell-1}+1, j_{\ell}}}{\left|j_{\ell}-j_{\ell-1}-2\right|}+\left|\frac{\sqrt{m} \bar{M}_{n}\left(j_{\ell-1}+1 ; j_{\ell}-1\right)}{j_{\ell}-j_{\ell-1}-2}\right|\right. \\
& \left.+\frac{\hat{w}_{j_{\ell}+1, j_{\ell+1}}}{\left|j_{\ell+1}-j_{\ell}-2\right|}+\left|\frac{\sqrt{m} \bar{M}_{n}\left(j_{\ell}+1 ; j_{\ell+1}-1\right)}{j_{\ell+1}-j_{\ell}-2}\right|\right\} \\
& \subset\left\{\left|\beta_{0, j_{\ell+1}-1, m}-\beta_{0, j_{k}-1, m}\right| \leq \frac{\hat{w}_{j_{\ell-1}+1, j_{\ell}}}{\Delta_{j, \text { min }}-2}+\left|\frac{\sqrt{m} \bar{M}_{n}\left(j_{\ell-1}+1 ; j_{\ell}-1\right)}{\Delta_{j, \text { min }}-2}\right|\right. \\
& \left.+\frac{\hat{w}_{j_{\ell}+1, j_{\ell+1}}}{\Delta_{j, \min }-2}+\left|\frac{\sqrt{m} \bar{M}_{n}\left(j_{\ell}+1 ; j_{\ell+1}-1\right)}{\Delta_{j, \min }-2}\right|\right\} \text {. }
\end{aligned}
$$


Then

$$
\begin{aligned}
\mathbb{P}\left[R_{n, \ell, 3}^{(4)}\right]= & \mathbb{P}\left[R_{n, \ell, 3}^{(4)} \cap Q_{n, \ell, 3}^{(4)}\right] \\
\leq \quad & \mathbb{P}\left[\frac{\hat{w}_{j_{\ell-1}+1, j_{\ell}}}{\Delta_{j, \min }-2} \geq \frac{\left|\beta_{0, j_{\ell+1}-1, m}-\beta_{0, j_{\ell}-1, m}\right|}{4}\right] \\
& +\mathbb{P}\left[\frac{\hat{w}_{j_{\ell}+1, j_{\ell+1}}}{\Delta_{j, \min }-2} \geq \frac{\left|\beta_{0, j_{\ell+1}-1, m}-\beta_{0, j_{\ell}-1, m}\right|}{4}\right] \\
& +\mathbb{P}\left[\left|\frac{\sqrt{m} \bar{M}_{n}\left(j_{\ell-1}+1 ; j_{\ell}-1\right)}{\Delta_{j, \min }-2}\right| \geq \frac{\left|\beta_{0, j_{\ell+1}-1, m}-\beta_{0, j_{\ell}-1, m}\right|}{4}\right] \\
& +\mathbb{P}\left[\left|\frac{\sqrt{m} \bar{M}_{n}\left(j_{\ell}+1 ; j_{\ell+1}-1\right)}{\Delta_{j, \min }-2}\right| \geq \frac{\left|\beta_{0, j_{\ell+1}-1, m}-\beta_{0, j_{\ell}-1, m}\right|}{4}\right] \\
\rightarrow \quad & 0 .
\end{aligned}
$$

as $n \rightarrow \infty$. This concludes the proof of Theorem 4 .

\section{Appendix A}

Here we prove Proposition 1 and Lemmas 1, 2 and 3

\section{A.1 Proof of Proposition 1}

Fix $j \in\{1, \ldots, m\}$. We have

$$
\begin{aligned}
& U_{j}=\frac{1}{n} \sum_{i=1}^{n} \int_{0}^{1} \mathbb{1}_{\left(\frac{j-1}{m}, 1\right]}(s) d M_{i}(s), \\
& V_{j}=n\left\langle U_{j}\right\rangle=\frac{1}{n} \int_{0}^{1} \mathbb{1}_{\left(\frac{j-1}{m}, 1\right]}(s) \lambda_{0}(s) d(s), \\
& \hat{V}_{j}=n\left[U_{j}\right]=\frac{1}{n} \sum_{i=1}^{n} \int_{0}^{1} \mathbb{1}_{\left(\frac{j-1}{m}, 1\right]}(s) d N_{i}(s) .
\end{aligned}
$$

Classical Bernstein deviation inequality applied to $U_{j}$, see [34], yields that

$$
\mathbb{P}\left[\left|U_{j}\right| \geq \sqrt{2 \theta z}+\frac{z}{3 n}, \frac{1}{n} \int_{0}^{1} \mathbb{1}_{\left(\frac{j-1}{m}, 1\right]}(s) \lambda_{0}(s) d(s) \leq \theta\right] \leq 2 e^{-z} .
$$

for all $\theta>0$, and $z>0$. It follows that

$$
\mathbb{P}\left[\left|U_{j}\right| \geq \sqrt{\frac{2 \theta z}{n}}+\frac{z}{3 n}, V_{j} \leq \theta\right] \leq 2 e^{-z} .
$$

By choosing $\theta=c_{0}(z+1) / n$, this gives

$$
\mathbb{P}\left[\left|U_{j}\right| \geq\left(\sqrt{2 c_{0}}+\frac{1}{3}\right) \frac{z+1}{n}, V_{j} \leq \frac{c_{0}(z+1)}{n}\right] \leq 2 e^{-z} .
$$

For any $0<\eta<\theta<\infty$, we have

$$
\left\{\left|U_{j}\right| \geq \sqrt{\frac{2 \theta V_{j} z}{\eta n}}+\frac{z}{3 n}\right\} \cap\left\{\eta<V_{j} \leq \theta\right\} \subset\left\{\left|U_{j}\right| \geq \sqrt{\frac{2 \theta z}{n}}+\frac{z}{3 n}\right\} \cap\left\{\eta<V_{j} \leq \theta\right\} .
$$


Together with (A.2), we obtain

$$
\mathbb{P}\left[\left|U_{j}\right| \geq \sqrt{\frac{2 \theta V_{j} z}{\eta n}}+\frac{z}{3 n}, \eta<V_{j} \leq \theta\right] \leq 2 e^{-z} .
$$

Now we want to replace $V_{j}$ by the observable $\hat{V}_{j}$ in the deviation (A.2). Define $\widetilde{U}_{j}$ by

$$
\begin{aligned}
\widetilde{U}_{j} & =\hat{V}_{j}-V_{j} \\
& =\frac{1}{n} \sum_{i=1}^{n} \int_{0}^{1} \mathbb{1}_{\left(\frac{j-1}{m}, 1\right]}(s) d M_{i}(s) .
\end{aligned}
$$

Now writing again (A.4) and using the same argument as before, we arrive at

$$
\mathbb{P}\left[\left|\widetilde{U}_{j}\right| \geq \sqrt{\frac{2 \theta V_{j} z}{\eta n}}+\frac{z}{3 n}, \eta<V_{j} \leq \theta\right] \leq 2 e^{-z} .
$$

But, if $V_{j}$ satisfies

$$
\left|\widetilde{U}_{j}\right| \leq \sqrt{\frac{2 \theta V_{j} z}{\eta n}}+\frac{z}{3 n}
$$

then it satisfies

$$
V_{j} \leq 2 \hat{V}_{j}+2\left(\frac{\theta}{\eta}+\frac{1}{3}\right) \frac{z}{n}
$$

and

$$
\hat{V}_{j} \leq 2 V_{j}+2\left(\frac{1}{3}+2 \sqrt{\frac{\theta}{\eta}\left(\frac{\theta}{\eta}+\frac{1}{3}\right)}+2 \frac{\theta}{\eta}\right) \frac{z}{n},
$$

simply using the fact that $A \leq b+\sqrt{a A}$ entails $A \leq a+2 b$ for any $a, A, b>0$. This proves that

$$
\begin{aligned}
\left\{\left|U_{j}\right|\right. & \left.\leq \sqrt{\frac{2 \theta V_{j} z}{\eta n}}+\frac{z}{3 n}\right\} \cap\left\{\left|\widetilde{U}_{j}\right| \leq \sqrt{\frac{2 \theta V_{j} z}{\eta n}}+\frac{z}{3 n}\right\} \\
& \subset\left\{\left|U_{j}\right| \leq 2 \sqrt{\frac{\theta z}{\eta n} \hat{V}_{j}}+\left(\frac{1}{3}+2 \sqrt{\frac{\theta}{\eta}\left(\frac{\theta}{\eta}+\frac{1}{3}\right)}+2 \frac{\theta}{\eta}\right) \frac{z}{n}\right\} .
\end{aligned}
$$

So, using (A.4) and (A.5), we obtain

$$
\mathbb{P}\left[\left|U_{j}\right| \geq 2 \sqrt{\frac{\theta z}{n} \hat{V}_{j}}+\left(\frac{1}{3}+2 \sqrt{\frac{\theta}{\eta}\left(\frac{\theta}{\eta}+\frac{1}{3}\right)}\right) \frac{z}{n}, \eta<V_{j} \leq \theta\right] \leq 4 e^{-z} .
$$

The inequality is similar to (A.4), where we replaced $V_{j}$ by the observable $\hat{V}_{j}$. It remains to remove the event $\left\{\eta<V_{j} \leq \theta\right\}$ from this inequality. First, recall that (A.3) holds, so we can work on the event $\left\{V_{j}>c_{0}(z+1) / n\right\}$ from now on. We use a peeling argument. Define, for $q \geq 0$ :

$$
\theta_{q}=c_{0} \frac{(z+1)}{n}(1+\varepsilon)^{q}
$$

and use the following decomposition into disjoint sets:

$$
\left\{V_{j}>\theta_{0}\right\}=\bigcup_{q \geq 0}\left\{\theta_{q}<V_{j} \leq \theta_{q+1}\right\} .
$$


We have

$$
\mathbb{P}\left[\left|U_{j}\right| \geq c_{1, \varepsilon} \sqrt{\frac{z}{n} \hat{V}_{j}}+c_{2, \varepsilon} \frac{z}{n}, \theta_{q}<V_{j} \leq \theta_{q+1}\right] \leq 4 e^{-z}
$$

where

$$
c_{1, \varepsilon}=2 \sqrt{1+\varepsilon} \quad \text { and } \quad c_{2, \varepsilon}=2 \sqrt{(1+\varepsilon)\left(\frac{4}{3}+\varepsilon\right)}+\frac{1}{3} \text {. }
$$

Let

$$
h_{j}=c_{h} \log \log \left(\frac{V_{j}}{\theta_{0}} \vee e\right) \text {. }
$$

On the event

$$
\left\{\left|\widetilde{U}_{j}\right| \leq \sqrt{\frac{2(1+\varepsilon) V_{j}\left(z+h_{j}\right)}{n}}+\frac{\left(z+h_{j}\right)}{3 n}\right\}
$$

we have

$$
V_{j} \leq 2 \hat{V}_{j}+2\left(\frac{4}{3}+\varepsilon\right) \frac{z}{n}+2 \frac{\frac{4}{3}+\varepsilon}{n} c_{h} \log \log \left(\frac{V_{j}}{\theta_{0}} \vee e\right),
$$

which entails, assuming that $e c_{0}>2\left((1+\varepsilon)+\frac{1}{3}\right) c_{h}$,

$$
V_{j} \leq \frac{e c_{0}(z+1)}{e c_{0}(z+1)-2\left(\frac{4}{3}+\varepsilon\right) c_{h}}\left(2 \hat{V}_{j}+2\left(\frac{4}{3}+\varepsilon\right) \frac{z}{n}\right),
$$

where we used the fact that $\log \log z \leq z / e-1$ for any $z \geq e$. This entails, together with (A.6), the following embedding:

$$
\begin{aligned}
\left\{\left|U_{j}\right|\right. & \left.\leq \sqrt{\frac{2(1+\varepsilon)\left(z+h_{j}\right) V_{j}}{n}}+\frac{z+h_{j}}{3 n}\right\} \cap\left\{\left|\widetilde{U}_{j}\right| \leq \sqrt{\frac{2(1+\varepsilon)\left(z+h_{j}\right) V_{j}}{n}}+\frac{\left(z+h_{j}\right)}{3 n}\right\} \\
& \subset\left\{\left|U_{j}\right| \geq c_{1, \varepsilon} \sqrt{\frac{z+\hat{h}_{n, z, j}}{n}} \hat{V}_{j}+c_{2, \varepsilon} \frac{z+\hat{h}_{n, z, j}}{n}\right\},
\end{aligned}
$$

where

$$
\hat{h}_{n, z, j}=c_{h} \log \log \left(\frac{2 e n \hat{V}_{j}+2 e\left(\frac{4}{3}+\varepsilon\right) z}{e c_{0}(z+1)-2\left(\frac{4}{3}+\varepsilon\right) c_{h}} \vee e\right) .
$$

Now, using the previous embeddings together with (A.4) and (A.5) we obtain

$$
\begin{aligned}
\mathbb{P}\left[\left|U_{j}\right| \geq\right. & \left.c_{1, \varepsilon} \sqrt{\frac{z+\hat{h}_{n, z, j}}{n} \hat{V}_{j}+c_{2, \varepsilon} \frac{z+\hat{h}_{n, z, j}}{n}}, V_{j}>\theta_{0}\right] \\
\leq & \sum_{q \geq 0} \mathbb{P}\left[\left|U_{j}\right| \geq \sqrt{\frac{2(1+\varepsilon) V_{j}\left(z+h_{j}\right)}{n}}+\frac{z+h_{j}}{3 n}, \theta_{q}<V_{j} \leq \theta_{q+1}\right] \\
& \quad+\sum_{q \geq 0} \mathbb{P}\left[\left|\widetilde{U}_{j}\right| \geq \sqrt{\frac{2(1+\varepsilon) V_{j}\left(z+h_{j}\right)}{n}}+\frac{z+h_{j}}{3 n}, \theta_{q}<V_{j} \leq \theta_{q+1}\right] \\
\leq & 4\left(e^{-z}+\sum_{q \geq 1} e^{-\left(z+c_{h} \log \log \left(\frac{V_{j}}{\theta_{0}}\right)\right)}\right) \\
= & 4\left(1+(\log (1+\varepsilon))^{-c_{h}} \sum_{q \geq 1} q^{-c_{h}}\right) e^{-z} .
\end{aligned}
$$

Then with (A.3), it implies that

$$
\mathbb{P}\left[\left|U_{j}\right| \geq c_{1, \varepsilon} \sqrt{\frac{z+\hat{h}_{n, z, j}}{n} \hat{V}_{j}}+c_{3, \varepsilon} \frac{z+1+\hat{h}_{n, z, j}}{n}\right] \leq\left(6+4(\log (1+\varepsilon))^{-c_{h}} \sum_{q \geq 1} q^{-c_{h}}\right) e^{-z},
$$

where $c_{3, \varepsilon}=\sqrt{2 \max \left(c_{0}, 2(1+\varepsilon)\left(\frac{4}{3}+\varepsilon\right)\right)}+\frac{1}{3}$. 


\section{A.2 Proof of Lemma 1}

Using the fact that the functions $\left\{\lambda_{j, m}: j=1, \ldots, m\right\}$ form a basis of $\Lambda_{m}$, and under Assumption 1, one can give the explicit form of $\lambda_{0, m}$ as following

$$
\lambda_{0, m}=m \sum_{j=1}^{m} \sum_{\ell=1}^{L_{0}} \beta_{0, \ell}\left|J_{\ell} \cap I_{j, m}\right| \mathbb{1}_{I_{j, m}}=\sum_{j=1}^{m} \beta_{0, j, m} \lambda_{j, m},
$$

here $|A|$ is the Lebesgue measure of the set $A$ and $\beta_{0, j, m}=\sqrt{m} \sum_{\ell=1}^{L_{0}} \beta_{0, \ell}\left|J_{\ell} \cap I_{j, m}\right|$. We remark that the intervals $J_{\ell}$ do not share the same boundaries as the smaller intervals $I_{j, m}$. Setting the sequence $\left(\bar{\ell}_{j}\right)_{j=0, \ldots, m}$ be the sequence defining by:

$$
\bar{\ell}_{0}=1, \text { and } \bar{\ell}_{j}=\max \left\{\ell=1, \ldots, L_{0}: J_{\ell} \cap I_{j, m} \neq \emptyset\right\} \text {, for } j=1, \ldots, m \text {. }
$$

Using the sequence $\left(\bar{\ell}_{j}\right)_{j=0, \ldots, m}$, one has the expression of the functions $\lambda_{0}$ and $\lambda_{0, m}$ as follows:

$$
\lambda_{0}=\sum_{j=1}^{m} \sum_{\ell=\bar{\ell}_{j-1}}^{\bar{\ell}_{j}} \beta_{0, \ell} \mathbb{1}_{J_{\ell} \cap I_{j, m}}
$$

and

$$
\lambda_{0, m}=m \sum_{j=1}^{m} \sum_{\ell=\bar{\ell}_{j-1}}^{\bar{\ell}_{j}} \beta_{0, \ell}\left|J_{\ell} \cap I_{j, m}\right| \mathbb{1}_{I_{j, m}}=\sum_{j=1}^{m} \alpha_{0, j, m} \mathbb{1}_{I_{j, m}}=\sum_{j=1}^{m} \alpha_{0, j, m} \sum_{\ell=\bar{\ell}_{j-1}}^{\bar{\ell}_{j}} \mathbb{1}_{J_{\ell} \cap I_{j, m}},
$$

where $\alpha_{0, j, m}=m \sum_{\ell=\bar{\ell}_{j-1}}^{\bar{\ell}_{j}} \beta_{0, \ell}\left|J_{\ell} \cap I_{j, m}\right|$. From the fact that $\left\{\mathbb{1}_{J_{\ell} \cap I_{j, m}}: j=1, \ldots, m\right.$ and $\ell=$ $\left.1, \ldots, L_{0}\right\}$ is an orthogonal basis of $\Lambda_{m}$ (with respect to the $\mathbb{L}^{2}$-norm), we obtain

$$
\begin{aligned}
\left\|\lambda_{0}-\lambda_{0, m}\right\|^{2} & =\left\|\sum_{j=1}^{m} \sum_{\ell=\bar{\ell}_{j-1}}^{\bar{\ell}_{j}}\left(\beta_{0, \ell}-m \sum_{\ell^{\prime}=\bar{\ell}_{j-1}}^{\bar{\ell}_{j}} \beta_{0, \ell^{\prime}}\left|J_{\ell^{\prime}} \cap I_{j, m}\right|\right) \mathbb{1}_{J_{\ell} \cap I_{j, m}}\right\|^{2} \\
& =\sum_{j=1}^{m} \sum_{\ell=\bar{\ell}_{j-1}}^{\bar{\ell}_{j}}\left(\beta_{0, \ell}-m \sum_{\bar{\ell}^{\prime}=\bar{\ell}_{j-1}}^{\bar{\ell}_{j}} \beta_{0, \ell^{\prime}}\left|J_{\ell^{\prime}} \cap I_{j, m}\right|\right)^{2}\left|J_{\ell} \cap I_{j, m}\right| \\
& =\sum_{j=1}^{m} \mathbb{1}_{\left[\bar{\ell}_{j}-\bar{\ell}_{j-1}>0\right]} \sum_{\ell=\bar{\ell}_{j-1}}^{\bar{\ell}_{j}}\left(\beta_{0, \ell}-m \sum_{\ell^{\prime}=\bar{\ell}_{j-1}}^{\bar{\ell}_{j}} \beta_{0, \ell^{\prime}}\left|J_{\ell^{\prime}} \cap I_{j, m}\right|\right)^{2}\left|J_{\ell} \cap I_{j, m}\right| \\
& \leq \sum_{j=1}^{m} \mathbb{1}_{\left[\bar{\ell}_{j}-\bar{\ell}_{j-1}>0\right]} \sum_{\ell=\bar{\ell}_{j-1}}^{\bar{\ell}_{j}}\left(\bar{\ell}_{j}-\bar{\ell}_{j-1}+1\right)_{\ell, \ell^{\prime}=k_{j-1}, \ldots, k_{j}}\left(\beta_{0, \ell}-\beta_{0, \ell^{\prime}}\right)^{2}\left|J_{\ell} \cap I_{j, m}\right| \\
& \leq \sum_{j=1}^{m} \mathbb{1}_{\left[\bar{\ell}_{j}-\bar{\ell}_{j-1}>0\right]}\left(\bar{\ell}_{j}-\bar{\ell}_{j-1}+1\right) \max _{\ell, \ell^{\prime} \in\left\{\bar{\ell}_{j-1}, \ldots, \bar{\ell}_{j}\right\}}\left(\beta_{0, \ell}-\beta_{0, \ell^{\prime}}\right)^{2}\left|I_{j, m}\right| \\
& \leq \frac{2\left(L_{0}-1\right) \Delta_{\beta, \max }^{2} .}{m} .
\end{aligned}
$$

This proves Lemma 1. 


\section{A.3 Proof of Lemma 2}

To prove Lemma 2, we invoke subdifferential calculus, see [5]. We first write our objective functional as

$$
\Phi(\mu)=\frac{1}{2} \sum_{j=1}^{m}\left(\mathbf{N}_{j}-(\mathbf{T} \mu)_{j}\right)^{2}+\sum_{j=1}^{m} \hat{w}_{j}\left|\mu_{j, m}\right| .
$$

So a necessary and sufficient condition for a vector $\hat{\mu}$ in $\mathbb{R}^{m}$ to minimize the function $\Phi$ is that the zero vector in $\mathbb{R}^{m}$ belongs to the sub-differential of $\Phi(\mu)$ at the point $\hat{\mu}$, that is, the following optimality condition holds:

$$
\text { for all } j=1, \ldots, m\left\{\begin{array}{l}
\left(\mathbf{T}^{\top}(\mathbf{N}-\mathbf{T} \hat{\mu})\right)_{j}=\hat{w}_{j} \operatorname{sign}\left(\hat{\mu}_{j, m}\right), \text { if } \hat{\mu}_{j, m} \neq 0, \\
\left|\left(\mathbf{T}^{\top}(\mathbf{N}-\mathbf{T} \hat{\mu})\right)_{j}\right| \leq \hat{w}_{j} \operatorname{sign}\left(\hat{\mu}_{j, m}\right), \text { if } \hat{\mu}_{j, m}=0 .
\end{array}\right.
$$

Using that $\left(\mathbf{T}^{\top} \mathbf{N}\right)_{j}=\sum_{q=j}^{m} \mathbf{N}_{q}$ and that $\left(\mathbf{T}^{\top} \hat{\beta}\right)_{j}=\sum_{q=j}^{m} \hat{\beta}_{q, m}$, since $\mathbf{T}$ is a $m \times m$ lower triangular matrix having all its nonzero elements equal to one. Now, for $q=1, \ldots, m$, we observe that

$$
\begin{aligned}
\mathbf{N}_{q}= & \sqrt{m} \int_{I_{q, m}} \lambda_{0}(t) d t+\sqrt{m} \bar{M}_{n}\left(I_{q, m}\right) \\
= & \sqrt{m} \int_{I_{q, m}}\left(\lambda_{0}(t)-\lambda_{0, m}(t)\right) d t+\sqrt{m} \int_{I_{q, m}} \lambda_{0, m} d t+\sqrt{m} \bar{M}_{n}\left(I_{q, m}\right) \\
= & \sqrt{m} \int_{I_{q, m}}\left(\sum_{\ell=1}^{L_{0}} \beta_{0, \ell} \mathbb{1}_{J_{\ell}}-\sqrt{m} \sum_{j=1}^{m} \beta_{0, j, m} \mathbb{1}_{I_{j, m}}\right) d t \\
& \quad+\sqrt{m} \sum_{j=1}^{m} \beta_{0, j, m} \int_{I_{q, m}} \mathbb{1}_{I_{j, m}}(t) d t+\sqrt{m} \bar{M}_{n}\left(I_{q, m}\right) \\
& \quad \beta_{0, q, m}+\sqrt{m} \bar{M}_{n}\left(I_{q, m}\right),
\end{aligned}
$$

and we get the desired result.

\section{A.4 Proof of Lemma 3}

For the first statement, we have by definition,

$$
\begin{aligned}
\left|\bar{M}_{n}(a ; b)\right| & =\left|\sum_{q=a}^{b} \bar{M}_{n}\left(I_{q, m}\right)\right| \\
& =\left|\frac{1}{n} \sum_{i=1}^{n} \sum_{q=a}^{b} \int_{0}^{1} I_{q, m}(t) d M_{i}(t)\right| \\
& =\left|\frac{1}{n} \sum_{i=1}^{n} \int_{0}^{1} \mathbf{1}_{\left(\frac{a-1}{m}, \frac{b}{m}\right]}(t) d M_{i}(t)\right| .
\end{aligned}
$$

Moreover, using Bernstein's inequality, it follows that, for any $z, \alpha>0$,

$$
\begin{aligned}
\mathbb{P}\left[\left|\frac{1}{n} \sum_{i=1}^{n} \int_{0}^{1} \mathbf{1}_{\left(\frac{a-1}{m}, \frac{b}{m}\right]}(t) d M_{i}(t)\right| \geq z\right. \\
\\
\left.\quad\left\langle\frac{1}{n} \sum_{i=1}^{n} \int_{0}^{1} \mathbf{1}_{\left(\frac{a-1}{m}, \frac{b}{m}\right]}(t) d M_{i}(t)\right\rangle \leq \alpha\right] \leq 2 \exp \left\{-\frac{z^{2}}{2 \alpha+\frac{2}{3} \rho z}\right\},
\end{aligned}
$$


where $\rho$ is a upper bound of $\left\|\frac{1}{n} \mathbf{1}_{\left(\frac{a-1}{m}, \frac{b}{m}\right]}\right\|_{\infty}$. Here we can choose $\rho=\frac{1}{n}$ and

$$
\alpha=n^{-1} \int_{\frac{a-1}{m}}^{\frac{b}{m}} \lambda_{0}(t) d t=n^{-1} \mathbb{E}\left[\bar{N}_{n}\left(\left(\frac{a-1}{m}, \frac{b}{m}\right]\right)\right] .
$$

Hence, we obtain the first statement. For the second one, recall that for any $a=2, \ldots, m$ we have

$$
\hat{w}_{a}=c_{1} \sqrt{\frac{m\left(x+\log m+\hat{h}_{n, x, a}\right) \hat{V}_{a}}{n}}+c_{2} \frac{\sqrt{m}\left(x+1+\log m+\hat{h}_{n, x, a}\right)}{n} .
$$

Since each term of $\hat{w}_{a}$ is positive and taking in account the dominant one, we have

$$
\left\{\hat{w}_{a}^{2} \geq \frac{m \log m}{n}\left(\xi-\int_{\mathbb{1}_{\left(\frac{a-1}{m}, 1\right]}} \lambda_{0}(t) d t\right)\right\} \subset\left\{\hat{V}_{a} \geq \xi-\int_{\mathbb{1}_{\left(\frac{a-1}{m}, 1\right]}} \lambda_{0}(t) d t\right\},
$$

for all $\xi>0$. By the Doob-Meyer decomposition theorem, we get

$$
\left\{\hat{w}_{a}^{2} \geq \frac{m \log m}{n}\left(\xi-\int_{\mathbb{1}_{\left(\frac{a-1}{m}, 1\right]}} \lambda_{0}(t) d t\right)\right\} \subset\left\{\bar{M}_{n}(a ; 1) \geq \xi\right\},
$$

Finally, by applying the first statement, see $(33)$, to $\bar{M}_{n}(a ; 1)$, we concludes the proof of Lemma 3.

\section{Appendix B}

Here we prove the second case of the proof of Theorem 3 which is quite similar to the first one with a careful choice of the bounded terms in the approximate change-points sequence while applying the KKT optimality conditions. As $m \varepsilon_{n} \geq 6$ for all $n \geq 1$, it yields that the event $\left\{j_{\ell}+2<\hat{j}_{\ell}\right\}$ a.s.

B.1 Step II.1. Prove: $\mathbb{P}\left[A_{n, \ell} \cap C_{n}\right] \rightarrow 0$, as $n \rightarrow \infty$.

Applying (31) in Lemma 2 with $j=j_{\ell}+1$ and $j=\hat{j}_{\ell}$, we get

$$
-\hat{w}_{j_{\ell}} \leq \sum_{q=j_{\ell}+1}^{m} \mathbf{N}_{q}-\sum_{q=j_{\ell}}^{m} \hat{\beta}_{q, m} \leq \hat{w}_{j_{\ell}+1}
$$

and

$$
-\hat{w}_{\hat{j}_{\ell}} \leq \sum_{q=\hat{j}_{\ell}}^{m} \mathbf{N}_{q}-\sum_{q=\hat{j}_{\ell}}^{m} \hat{\beta}_{q, m} \leq \hat{w}_{\hat{j}_{\ell}}
$$

It follows that

$$
\left|\sum_{q=j_{\ell}+1}^{\hat{j}_{\ell}-1} \beta_{0, q, m}+\sqrt{m} \bar{M}_{n}\left(I_{q, m}\right)-\sum_{q=j_{\ell}+1}^{\hat{j}_{\ell}-1} \hat{\beta}_{q, m}\right| \leq \hat{w}_{j_{\ell}+1, \hat{j}_{\ell}} .
$$

The property of the vector $\hat{\beta}$ in Lemma 2 yields that

$$
\left|\left(\hat{j}_{\ell}-j_{\ell}-2\right)\left(\beta_{0, j_{\ell+1}-1, m}-\hat{\beta}_{\hat{j}_{\ell}-1, m}\right)+\sqrt{m} \bar{M}_{n}\left(j_{\ell}+1 ; \hat{j}_{\ell}-1\right)\right| \leq \hat{w}_{j_{\ell}+1, \hat{j}_{\ell}} .
$$


Therefore, on $C_{n} \cap\left\{\hat{j}_{\ell}>j_{\ell}+2\right\}$ we have

$$
\begin{aligned}
& \mid\left(\hat{j}_{\ell}-j_{\ell}-2\right)\left(\beta_{0, j_{\ell}-1, m}-\hat{\beta}_{\hat{j}_{\ell}-1, m}\right) \\
& +\sqrt{m} \bar{M}_{n}\left(j_{\ell}+1 ; \hat{j}_{\ell}-1\right) \\
& \quad+\left(\hat{j}_{\ell}-j_{\ell}-2\right)\left(\beta_{0, j_{\ell+1}-1, m}-\beta_{0, j_{\ell}-1, m}\right) \mid \leq \hat{w}_{j_{\ell}+1, \hat{j}_{\ell}} .
\end{aligned}
$$

Define the event

$$
\begin{aligned}
C_{n, \ell}^{\prime}=\left\{\mid\left(\hat{j}_{\ell}-j_{\ell}-2\right)\left(\beta_{0, j_{\ell}-1, m}-\hat{\beta}_{\hat{j}_{\ell}-1, m}\right)\right. & \\
& +\sqrt{m} \bar{M}_{n}\left(j_{\ell}+1 ; \hat{j}_{\ell}-1\right) \\
& \left.+\left(\hat{j}_{\ell}-j_{\ell}-2\right)\left(\beta_{0, j_{\ell+1}-1, m}-\beta_{0, j_{\ell}-1, m}\right) \mid \leq \hat{w}_{j_{\ell}+1, \hat{j}_{\ell}}\right\} .
\end{aligned}
$$

It follows that $C_{n, \ell}^{\prime}$ occurs with probability one. We observe that, $m \varepsilon_{n} \geq 6$ for all $n$, entails that $\frac{m \varepsilon_{n}}{2}-2 \geq \frac{m \varepsilon_{n}}{6}$. Then

$$
\left\{\left|\hat{j}_{\ell}-j_{\ell}\right|>\frac{m \varepsilon_{n}}{2}\right\} \subset\left\{\left|\hat{j}_{\ell}-j_{\ell}-2\right|>\frac{m \varepsilon_{n}}{2}-2\right\} \subset\left\{\left|\hat{j}_{\ell}-j_{\ell}-2\right| \geq \frac{m \varepsilon_{n}}{6}\right\} .
$$

Therefore

$$
\begin{aligned}
& \mathbb{P}\left[A_{n, \ell} \cap C_{n}\right] \\
& =\mathbb{P}\left[\left\{\mid\left(\hat{j}_{\ell}-j_{\ell}-2\right)\left(\beta_{0, j_{\ell}-1, m}-\hat{\beta}_{\hat{j}_{\ell}-1, m}\right)+\sqrt{m} \bar{M}_{n}\left(j_{\ell}+1 ; \hat{j}_{\ell}-1\right)\right.\right. \\
& \left.+\left(\hat{j}_{\ell}-j_{\ell}-2\right)\left(\beta_{0, j_{\ell+1}-1, m}-\beta_{0, j_{\ell}-1, m}\right) \mid \leq \hat{w}_{j_{\ell}+1, \hat{j}_{\ell}}\right\} \\
& \left.\cap A_{n, \ell} \cap C_{n} \cap\left\{\hat{j}_{\ell}>j_{\ell}+2\right\}\right] \\
& \leq \mathbb{P}\left[\left\{\frac{\hat{w}_{j_{\ell}+1, \hat{j}_{\ell}}}{\frac{m \varepsilon_{n}}{6}} \geq \frac{\left|\beta_{0, j_{\ell+1}-1, m}-\beta_{0, j_{\ell}-1, m}\right|}{3}\right\} \cap\left\{\hat{j}_{\ell}>j_{\ell}+2\right\}\right] \\
& +\mathbb{P}\left[\left\{\left|\hat{\beta}_{\hat{j}_{\ell}-1, m}-\beta_{0, j_{\ell}-1, m}\right| \geq \frac{\left|\beta_{0, j_{\ell+1}-1, m}-\beta_{0, j_{\ell}-1, m}\right|}{3}\right\} \cap C_{n}\right] \\
& +\mathbb{P}\left[\left|\frac{\sqrt{m} \bar{M}_{n}\left(j_{\ell} ; \hat{j}_{\ell}-1\right)}{\hat{j}_{\ell}-j_{\ell}-2}\right| \geq \frac{\left|\beta_{0, j_{\ell+1}-1, m}-\beta_{0, j_{\ell}-1, m}\right|}{3}\right] \\
& :=\mathbb{P}\left[A_{n, \ell, 1}^{\prime}\right]+\mathbb{P}\left[A_{n, \ell, 2}^{\prime}\right]+\mathbb{P}\left[A_{n, \ell, 3}^{\prime}\right] \text {. }
\end{aligned}
$$

We have

$$
\begin{aligned}
\mathbb{P}\left[A_{n, \ell, 1}^{\prime}\right] & \leq \mathbb{P}\left[\hat{w}_{j_{\ell}+1, \hat{j}_{\ell}} \geq \frac{m \varepsilon_{n} \Delta_{\beta, \min }}{18}\right] \\
& \leq \mathbb{P}\left[\hat{w}_{j_{\ell}+1} \geq \frac{m \varepsilon_{n} \Delta_{\beta, \min }}{36}\right] \\
& =\mathbb{P}\left[\hat{w}_{j_{\ell}+1}^{2} \geq \frac{m^{2} \varepsilon_{n}^{2} \Delta_{\beta, \min }^{2}}{36^{2}}\right]
\end{aligned}
$$

By (16) in Assumption 4, and (34) in Lemma 3 with $\xi=\frac{n m \varepsilon_{n}^{2} \Delta_{\beta, \min }^{2}}{36^{2} \log m}+\mathbb{E}\left[\bar{N}_{n}\left(\left(\frac{j_{\ell}}{m}, 1\right]\right)\right]$, it follows that

$$
\mathbb{P}\left(A_{n, \ell, 1}^{\prime}\right) \leq 2 \exp \left(-\frac{n \xi^{2}}{2 \mathbb{E}\left[\bar{N}_{n}\left(\left(\frac{j_{\ell}}{m}, 1\right]\right)\right]+\frac{2}{3} \xi}\right) \rightarrow 0
$$


as $n \rightarrow \infty$. Now, we remark that

$$
A_{n, \ell, 3}^{\prime} \subset\left\{\left|\bar{M}_{n}\left(j_{\ell} ; \hat{j}_{\ell}-1\right)\right| \geq \frac{m \varepsilon_{n} \Delta_{\beta, \min }}{18 \sqrt{m}}\right\} \subset \bigcup_{q=j_{\ell}+2}^{j_{\ell+1}-2}\left\{\left|\bar{M}_{n}\left(j_{\ell} ; q\right)\right| \geq \frac{m \varepsilon_{n} \Delta_{\beta, \min }}{18 \sqrt{m}}\right\}
$$

Let $\varphi_{n}^{\prime}:=\frac{\sqrt{m} \varepsilon_{n} \Delta_{\beta, \min }}{18}$. Hence, by (33) in Lemma 3 we get

$$
\begin{aligned}
\mathbb{P}\left[A_{n, \ell, 3}^{\prime}\right] & \leq 2 \sum_{q=j_{\ell}+2}^{j_{\ell+1}-2} \exp \left(-\frac{n \varphi_{n}^{\prime 2}}{2 \mathbb{E}\left[\bar{N}_{n}\left(\left(\frac{j_{\ell}-1}{m}, \frac{q}{m}\right]\right)\right]+\frac{2}{3} \varphi_{n}^{\prime}}\right) \\
& \leq 2\left(j_{\ell+1}-j_{\ell}-3\right) \exp \left(-\frac{n \varphi_{n}^{\prime 2}}{2 \mathbb{E}\left[\bar{N}_{n}\left(\left(\frac{j_{\ell}-1}{m}, \frac{j_{\ell+1}-2}{m}\right]\right)\right]+\frac{2}{3} \varphi_{n}^{\prime}}\right) \\
& \leq 2 \exp \left(-\frac{n \varphi_{n}^{\prime 2}}{2 \mathbb{E}\left[\bar{N}_{n}\left(\left(\frac{j_{\ell}-1}{m}, \frac{j_{\ell+1}-2}{m}\right]\right)\right]+\frac{2}{3} \varphi_{n}^{\prime}}+\log m\right) .
\end{aligned}
$$

By (16) in Assumption 4, we get $\mathbb{P}\left[A_{n, \ell, 3}^{\prime}\right]$ tends to zero as $n$ tends to infinity. Let us now address $\mathbb{P}\left[A_{n, \ell, 2}^{\prime}\right]$. Using (31) in Lemma 2 with $j=j_{\ell}$ and with $j=\left\lceil\frac{j_{\ell}+j_{\ell-1}}{2}\right\rceil$, and using the triangle inequality, it follows that

$$
\left|\sum_{q=\left\lceil\frac{j_{\ell}+j_{\ell-1}}{2}\right\rceil}^{j_{\ell}-1} \mathbf{N}_{q}-\sum_{q=\left\lceil\frac{j_{\ell}+j_{\ell-1}}{2}\right\rceil}^{j_{\ell}-1} \hat{\beta}_{q, m}\right| \leq \hat{w}_{\left\lceil\frac{j_{\ell}+j_{\ell-1}}{2}\right\rceil, j_{\ell}} .
$$

On the event $C_{n} \cap\left\{\hat{j}_{\ell}>j_{\ell}\right\}$, we get

$$
\left|\frac{j_{\ell}-j_{\ell-1}}{2}\left(\beta_{0, j_{\ell}-1, m}-\hat{\beta}_{\hat{j}_{\ell}-1, m}\right)+\sqrt{m} \bar{M}_{n}\left(\left\lceil\frac{j_{\ell}+j_{\ell-1}}{2}\right\rceil ; j_{\ell}-1\right)\right| \leq \hat{w}_{\left\lceil\frac{j_{\ell}+j_{\ell-1}}{2}\right\rceil, j_{\ell}} .
$$

This implies

$$
\left|\frac{j_{\ell}-j_{\ell-1}}{2}\right|\left|\hat{\beta}_{\hat{j}_{\ell}-1, m}-\beta_{0, j_{\ell}-1, m}\right| \leq \hat{w}_{\left\lceil\frac{j_{\ell}+j_{\ell-1}}{2}\right\rceil, j_{\ell}}+\left|\sqrt{m} \bar{M}_{n}\left(\left\lceil\frac{j_{\ell}+j_{\ell-1}}{2}\right\rceil ; j_{\ell}-1\right)\right| .
$$

Therefore, we may upper bound $\mathbb{P}\left[A_{n, \ell, 2}^{\prime}\right]$ as follows

$$
\begin{aligned}
& \mathbb{P}\left[A_{n, \ell, 2}^{\prime}\right] \mathbb{P}\left[\left\{\left|\hat{\beta}_{\hat{j}_{\ell}-1, m}-\beta_{0, j_{\ell}-1, m}\right| \geq \frac{\left|\beta_{0, j_{\ell+1}-1, m}-\beta_{0, j_{\ell}-1, m}\right|}{3}\right\} \cap C_{n} \cap\left\{\hat{j}_{\ell}>j_{\ell}\right\}\right] \\
&= \mathbb{P}\left[\left\{\left|\frac{j_{\ell}-j_{\ell-1}}{2}\right|\left|\hat{\beta}_{\hat{j}_{\ell}-1, m}-\beta_{0, j_{\ell}-1, m}\right|\right.\right. \\
&\left.\left.\quad \geq\left|\frac{j_{\ell}-j_{\ell-1}}{2}\right| \frac{\left|\beta_{0, j_{\ell+1}-1, m}-\beta_{0, j_{\ell}-1, m}\right|}{3}\right\} \cap C_{n}\right] \\
& \leq \mathbb{P}\left[\left\{\hat{w}_{\left\lceil\frac{j_{\ell}+j_{\ell-1}}{2}\right\rceil, j_{\ell}}+\left|\sqrt{m} \bar{M}_{n}\left(\left\lceil\frac{j_{\ell}+j_{\ell-1}}{2}\right\rceil ; j_{\ell}-1\right)\right|\right.\right. \\
&\left.\left.\quad \geq\left|\frac{j_{\ell}-j_{\ell-1}}{2}\right| \frac{\left|\beta_{0, j_{\ell+1}-1, m}-\beta_{0, j_{\ell}-1, m}\right|}{3}\right\} \cap C_{n}\right] \\
& \leq \mathbb{P}\left[\hat{w}_{\left\lceil\frac{j_{\ell}+j_{\ell-1}}{2}\right\rceil, j_{\ell}} \geq\left(j_{\ell}-j_{\ell-1}\right) \frac{\left|\beta_{0, j_{\ell+1}-1, m}-\beta_{0, j_{\ell}-1, m}\right|}{6}\right] \\
& \quad+\mathbb{P}\left[\left|\sqrt{m} \bar{M}_{n}\left(\left\lceil\frac{j_{\ell}+j_{\ell-1}}{2}\right\rceil ; j_{\ell}-1\right)\right| \geq \frac{\left|\beta_{0, j_{\ell+1}-1, m}-\beta_{0, j_{\ell}-1, m}\right|}{6}\right] \\
& \leq \mathbb{P}\left[\hat{w}_{\left\lceil\frac{j_{\ell}+j_{\ell-1}}{2}\right\rceil, j_{\ell}} \geq \frac{\Delta_{j, \min } \Delta_{\beta, \min }}{12}\right]+\mathbb{P}\left[\left|\bar{M}_{n}\left(\left\lceil\frac{j_{\ell}+j_{\ell-1}}{2}\right\rceil ; j_{\ell}-1\right)\right| \geq \frac{\Delta_{j, \min } \Delta_{\beta, \min }}{12 \sqrt{m}}\right] \\
&:= \alpha_{n, \ell, 2}^{\prime}{ }^{(1)}+\alpha_{n, \ell, 2}^{\prime}(2) .
\end{aligned}
$$


We observe that

$$
\alpha_{n, \ell, 2}^{\prime}{ }^{(1)} \leq \mathbb{P}\left[\hat{w}_{\left\lceil\frac{j_{\ell}+j_{\ell-1}}{2}\right\rceil}^{2} \geq \frac{\Delta_{j, \min }^{2} \Delta_{\beta, \min }^{2}}{24^{2}}\right] \leq \mathbb{P}\left[\hat{w}_{j_{\ell-1}}^{2} \geq \frac{\Delta_{j, \min }^{2} \Delta_{\beta, \min }^{2}}{24^{2}}\right] .
$$

By (17) in Assumption 4, (34) in Lemma 3 with $\xi=\frac{n \Delta_{j, \min }^{2} \Delta_{\beta, \min }^{2}}{24^{2} m \log m}+\mathbb{E}\left[\bar{N}_{n}\left(\left(\frac{j_{\ell-1}-1}{m}, 1\right]\right)\right]$, it follows that

$$
\alpha_{n, \ell, 2}^{\prime}{ }^{(1)} \leq 2 \exp \left(-\frac{n \xi^{2}}{2 \mathbb{E}\left[\bar{N}_{n}\left(\left(\frac{j_{\ell-1}-1}{m}, 1\right]\right)\right]+\frac{2}{3} \xi}\right) \rightarrow 0,
$$

as $n \rightarrow \infty$. By (33) in Lemma 3 with $z=\frac{\Delta_{j, \min } \Delta_{\beta, \min }}{12 \sqrt{m}}$ and (17) in Assumption 4, we obtain

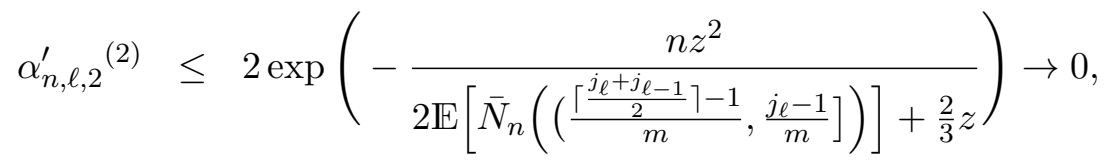

as $n \rightarrow \infty$. Therefore, we conclude that $\mathbb{P}\left[A_{n, \ell, 2}^{\prime}\right] \rightarrow 0$, as $n \rightarrow \infty$.

B.2 Step II.2. Prove: $\mathbb{P}\left[A_{n, \ell} \cap C_{n}^{\complement}\right] \rightarrow 0$, as $n \rightarrow \infty$.

As in Case I from 8.1, we split $\mathbb{P}\left[A_{n, \ell} \cap C_{n}^{\complement}\right]$ into

$$
\mathbb{P}\left[A_{n, \ell} \cap C_{n}^{\complement}\right]=\mathbb{P}\left[A_{n, \ell} \cap D_{n}^{(l)}\right]+\mathbb{P}\left[A_{n, \ell} \cap D_{n}^{(m)}\right]+\mathbb{P}\left[A_{n, \ell} \cap D_{n}^{(r)}\right] .
$$

Let us first focus on $\mathbb{P}\left[A_{n, \ell} \cap D_{n}^{(m)}\right]$. Note that

$$
\begin{aligned}
\mathbb{P}\left[A_{n, \ell} \cap D_{n}^{(m)} \cap\left\{\hat{j}_{\ell}>j_{\ell}\right\}\right] \leq & \mathbb{P}\left[A_{n, \ell} \cap B_{\ell+1, \ell} \cap D_{n}^{(m)}\right] \\
& +\sum_{l=\ell+1}^{L_{0}-2} \mathbb{P}\left[C_{l, l} \cap B_{l+1, l} \cap D_{n}^{(m)}\right] .
\end{aligned}
$$

Let us now prove that the first term in the right hand side of (B.1) goes to zero as $n$ tends to infinity. Using (31) in Lemma 2 with $j=\left\lceil\frac{j_{\ell+1}+j_{\ell}}{2}\right\rceil$ and $j=j_{\ell+1}$, on the first hand and (31) in Lemma 2 with $j=j_{\ell+1}+1$ and with $j=j_{\ell+2}$ on the other hand, we obtain, respectively

$$
\begin{aligned}
\left|\frac{j_{\ell+1}-j_{\ell}}{2}\right|\left|\hat{\beta}_{\hat{j}_{\ell+1}-1, m}-\beta_{0, j_{\ell+1}-1, m}\right| \leq \hat{w}_{\left\lceil\frac{j_{\ell+1}+j_{\ell}}{2}\right\rceil, j_{\ell+1}} & \\
& +\left|\sqrt{m} \bar{M}_{n}\left(\left\lceil\frac{j_{\ell+1}+j_{\ell}}{2}\right\rceil ; j_{\ell+1}-1\right)\right|,
\end{aligned}
$$

and

$$
\begin{aligned}
\left|j_{\ell+2}-j_{\ell+1}-2\right|\left|\hat{\beta}_{\hat{j}_{\ell+1}-1, m}-\beta_{0, j_{\ell+2}-1, m}\right| \leq & \hat{w}_{j_{\ell+1}+1, j_{\ell+2}} \\
& +\left|\sqrt{m} \bar{M}_{n}\left(j_{\ell+1}+1 ; j_{\ell+2}-1\right)\right| .
\end{aligned}
$$


In addition, we have

$$
\begin{aligned}
& \left|\beta_{0, j_{\ell+2}-1, m}-\beta_{0, j_{\ell+1}-1, m}\right| \\
& =\left|\left(\hat{\beta}_{\hat{j}_{\ell+1}-1, m}-\beta_{0, j_{\ell+1}-1, m}\right)-\left(\hat{\beta}_{\hat{j}_{\ell+1}-1, m}-\beta_{0, j_{\ell+2}-1, m}\right)\right| \\
& \leq\left|\hat{\beta}_{\hat{j}_{\ell+1}-1, m}-\beta_{0, j_{\ell+1}-1, m}\right|+\left|\hat{\beta}_{\hat{j}_{\ell+1}-1, m}-\beta_{0, j_{\ell+2}-1, m}\right|
\end{aligned}
$$

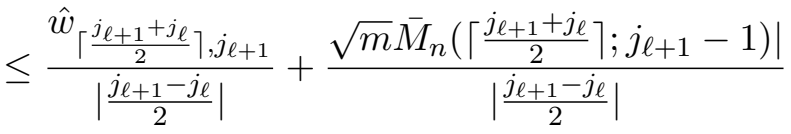

$$
\begin{aligned}
& +\frac{\hat{w}_{j_{\ell+1}+1, j_{\ell+2}}}{\left|j_{\ell+2}-j_{\ell+1}-2\right|}+\frac{\left|\sqrt{m} \bar{M}_{n}\left(j_{\ell+1}+1 ; j_{\ell+2}-1\right)\right|}{\left|j_{\ell+2}-j_{\ell+1}-2\right|}
\end{aligned}
$$

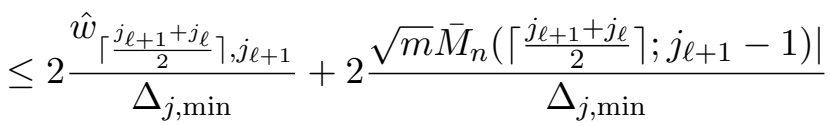

$$
\begin{aligned}
& +\frac{\hat{w}_{j_{\ell+1}+1, j_{\ell+2}}}{\left|\Delta_{j, \min }-2\right|}+\frac{\left|\sqrt{m} \bar{M}_{n}\left(j_{\ell+1}+1 ; j_{\ell+2}-1\right)\right|}{\left|\Delta_{j, \min }-2\right|} .
\end{aligned}
$$

Define the event $E_{n, \ell}^{\prime}$ by

$$
\begin{aligned}
E_{n, \ell}^{\prime}=\left\{\left|\beta_{0, j_{\ell+2}-1, m}-\beta_{0, j_{\ell+1}-1, m}\right| \leq\right. & \frac{\left.2 \hat{w}_{\left\lceil j_{\ell+1}+j_{\ell}\right.}\right\rceil, j_{\ell+1}}{\Delta_{j, \min }}+\frac{6 \hat{w}_{j_{\ell+1}+1, j_{\ell+2}}}{\Delta_{j, \min }} \\
& +\frac{2 \sqrt{m} \bar{M}_{n}\left(\left\lceil\frac{j_{\ell+1}+j_{\ell}}{2}\right\rceil ; j_{\ell+1}-1\right) \mid}{\Delta_{j, \min }} \\
& \left.+\frac{\left|6 \sqrt{m} \bar{M}_{n}\left(j_{\ell+1}+1 ; j_{\ell+2}-1\right)\right|}{\Delta_{j, \min }}\right\} .
\end{aligned}
$$

$E_{n, \ell}^{\prime}$ occurs with probability one. Therefore, we obtain

$$
\begin{aligned}
\mathbb{P}\left[A_{n, \ell}\right. & \left.\cap B_{\ell+1, \ell} \cap D_{n}^{(m)}\right] \\
\leq & \mathbb{P}\left[A_{n, \ell} \cap B_{\ell+1, \ell} \cap D_{n}^{(m)} \cap E_{n, \ell}^{\prime}\right] \\
\leq & \mathbb{P}\left[\hat{w}_{\left\lceil\frac{j_{\ell+1}+j_{\ell}}{2}\right\rceil, j_{\ell+1}} \geq \frac{\Delta_{j, \min }\left|\beta_{0, j_{\ell+2}-1, m}-\beta_{0, j_{\ell+1}-1, m}\right|}{8}\right] \\
& +\mathbb{P}\left[\hat{w}_{j_{\ell+1}+1, j_{\ell+2}} \geq \frac{\left|\beta_{0, j_{\ell+2}-1, m}-\beta_{0, j_{\ell+1}-1, m}\right|}{24}\right] \\
& +\mathbb{P}\left[\left|\bar{M}_{n}\left(\left\lceil\frac{j_{\ell+1}+j_{\ell}}{2}\right\rceil ; j_{\ell+1}-1\right)\right| \geq \Delta_{j, \min } \frac{\left|\beta_{0, j_{\ell+2}-1, m}-\beta_{0, j_{\ell+1}-1, m}\right|}{8 \sqrt{m}}\right] \\
& +\mathbb{P}\left[\left|\bar{M}_{n}\left(j_{\ell+1}+1 ; j_{\ell+2}-1\right)\right| \geq \Delta_{j, \min } \frac{\left|\beta_{0, j_{\ell+2}-1, m}-\beta_{0, j_{\ell+1}-1, m}\right|}{24 \sqrt{m}}\right] \\
:= & \theta_{n, \ell, 1}^{\prime}+\theta_{n, \ell, 2}^{\prime}+\theta_{n, \ell, 3}^{\prime}+\theta_{n, \ell, 4}^{\prime} .
\end{aligned}
$$

By (33)-(34) in Lemma 3, and (16)-(17) in Assumption 4, we show that for $s=1, \ldots, 4, \theta_{n, \ell, s}^{\prime} \rightarrow$ $0, \mathbb{P}\left[A_{n, \ell} \cap B_{\ell+1, \ell} \cap D_{n}^{(m)}\right] \rightarrow 0$, as $n \rightarrow \infty$. Recall that in Case I from Section 8.1, we proved $\mathbb{P}\left[A_{n, \ell} \cap D_{n}^{(l)}\right] \rightarrow 0$, as $n \rightarrow \infty$ and in a similar way $\mathbb{P}\left[A_{n, \ell} \cap D_{n}^{(r)}\right] \rightarrow 0$, as $n \rightarrow \infty$. This concludes the proof of Theorem 3 .

\section{References}

[1] O. Aalen. Nonparametric inference for a family of counting processes. Ann. Statist., 6(4):701$726,1978$. 
[2] P. K. Andersen, Ø. Borgan, R. D. Gill, and N. Keiding. Statistical models based on counting processes. Springer Series in Statistics. Springer-Verlag, New York, 1993.

[3] F. Bach, R. Jenatton, J. Mairal, and G. Obozinski. Optimization with sparsity-inducing penalties. Foundations and Trends® in Machine Learning, 4(1):1-106, 2012.

[4] H. H. Bauschke and P. L. Combettes. Convex analysis and monotone operator theory in Hilbert spaces. CMS Books in Mathematics/Ouvrages de Mathématiques de la SMC. Springer, New York, 2011.

[5] D. P. Bertsekas. Nonlinear Programming. Athena Scientific, Belmont, MA, 1999.

[6] P. J. Bickel, Y. Ritov, and A. B. Tsybakov. Simultaneous analysis of lasso and Dantzig selector. Ann. Statist., 37(4):1705-1732, 2009.

[7] K. Bleakley and J. P. Vert. The group fused Lasso for multiple change-point detection. 2011.

[8] S. Boyd and L. Vandenberghe. Convex optimization. Cambridge University Press, Cambridge, 2004.

[9] L. Boysen, A. Kempe, V. Liebscher, A. Munk, and O. Wittich. Consistencies and rates of convergence of jump-penalized least squares estimators. Ann. Statist., 37(1):157-183, 2009.

[10] P. Brémaud. Point processes and queues. Springer-Verlag, New York, 1981. Martingale dynamics, Springer Series in Statistics.

[11] A. Chambolle, V. Caselles, D. Cremers, M. Novaga, and T. Pock. An introduction to total variation for image analysis. Theoretical foundations and numerical methods for sparse recovery, 9:263-340, 2010.

[12] A. Chambolle and J. Darbon. On total variation minimization and surface evolution using parametric maximum flows. International journal of computer vision, 84(3):288-307, 2009.

[13] F. Chen, P. F. Yip, and K. F. Lam. On the local polynomial estimators of the counting process intensity function and its derivatives. Scand. J. Stat., 38(4):631-649, 2011.

[14] Derek Y Chiang, Gad Getz, David B Jaffe, Michael JT O'Kelly, Xiaojun Zhao, Scott L Carter, Carsten Russ, Chad Nusbaum, Matthew Meyerson, and Eric S Lander. High-resolution mapping of copy-number alterations with massively parallel sequencing. Nature methods, 6(1):99-103, 2009.

[15] G. Ciuperca. Model selection by LASSO methods in a change-point model. Statist. Papers, $55(2): 349-374,2014$.

[16] F. Comte, S. Gaïfas, and A. Guilloux. Adaptive estimation of the conditional intensity of marker-dependent counting processes. Ann. Inst. Henri Poincaré Probab. Stat., 47(4):11711196, 2011.

[17] L. Condat. A Direct Algorithm for 1D Total Variation Denoising. IEEE Signal Processing Letters, 20(11):1054-1057, 2013.

[18] S. Gaïffas and A. Guilloux. High-dimensional additive hazards models and the Lasso. Electron. J. Stat., 6:522-546, 2012.

[19] Melody S. Goodman, Yi Li, and Ram C. Tiwari. Detecting multiple change points in piecewise constant hazard functions. J. Appl. Stat., 38(11):2523-2532, 2011.

[20] G. Grégoire. Least squares cross-validation for counting process intensities. Scand. J. Statist., 20(4):343-360, 1993.

[21] Z. Harchaoui and C. Lévy-Leduc. Multiple change-point estimation with a total variation penalty. J. Amer. Statist. Assoc., 105(492):1480-1493, 2010.

[22] D. S. Hochbaum. An efficient algorithm for image segmentation, markov random fields and related problems. Journal of the ACM (JACM), 48(4):686-701, 2001.

[23] A.F. Karr. Point processes and their statistical inference, volume 7. CRC press, 1991. 
[24] A. Khodadadi and M. Asgharian. Change-point problems and regression: An annotated bibliography. Collection of Biostatistics Research Archive (COBRA), 2008.

[25] R. Sh. Liptser and A. N. Shiryayev. Theory of martingales, volume 49 of Mathematics and its Applications (Soviet Series). 1989.

[26] P. N. Patil and A. T. A. Wood. Counting process intensity estimation by orthogonal wavelet methods. Bernoulli, 10(1):1-24, 2004.

[27] F. Picard, S. Robin, E. Lebarbier, and J-J Daudin. A segmentation/clustering model for the analysis of array cgh data. Biometrics, 63(3):758-766, 2007.

[28] J. Qian and L. Su. Shrinkage estimation of common breaks in panel data models via adaptive group fused lasso, working paper, 2013.

[29] H. Ramlau-Hansen. Smoothing counting process intensities by means of kernel functions. Ann. Statist., 11(2):453-466, 1983.

[30] P. Reynaud-Bouret. Adaptive estimation of the intensity of inhomogeneous Poisson processes via concentration inequalities. Probab. Theory Related Fields, 126(1):103-153, 2003.

[31] A. Rinaldo. Properties and refinements of the fused lasso. Ann. Statist., 37(5B):2922-2952, 2009.

[32] J. J. Shen and N. R. Zhang. Change-point model on nonhomogeneous Poisson processes with application in copy number profiling by next-generation DNA sequencing. Ann. Appl. Stat., 6(2):476-496, 2012.

[33] R. Tibshirani, M. Saunders, S. Rosset, J. Zhu, and K. Knight. Sparsity and smoothness via the fused lasso. J. R. Stat. Soc. Ser. B Stat. Methodol., 67(1):91-108, 2005.

[34] S. Van De Geer. Exponential inequalities for martingales, with application to maximum likelihood estimation for counting processes. Ann. Statist., 23(5):1779-1801, 1995.

[35] D. Yu, J. Won, T. Lee, J. Lim, and S. Yoon. High-dimensional fused lasso regression using majorization-minimization and parallel processing. To appear in Journal of Computational and Graphical Statistics. 